\title{
Die Intensitäten der Starkregen in der Schweiz
}

\author{
Von A. HÖRIER und H. R. RHEIN \\ Eidg. Anstalt für Wasserversorgung, Abwasserreinigung und Gewässerschutz. \\ (EAWAG) an der ETH, Zürich \\ Dircktor: Prof. Dr. O. JAAG \\ Manuskript eingegangen am Io. Juli I962
}

Formelzeichen. . . . . . . . . . . . . . . . . . . 292

I. Finleitung . . . . . . . . . . . . . . . . . . . 294

2. Bisher in der Scbweiz permendete Regenintensitätskurven . . . . . . . . 295

3. Vorbandenes Beobacbtungsmaterial . . . . . . . . . . . . . . . . . . 298

3.I Vorhandene Schreibregenmesser . . . . . . . . . . . . . 298

3.2 Vorhandene Regenstreifen . . . . . . . . . . . . . . . . . . . 299

4. Das Auswerteverfabren . . . . . . . . . . . . . . . . . . . . . . . 299

4.I Verfahren von REINHOLD . . . . . . . . . . . . . . . . 299

4.2 Kritik am Verfahren von REINHOLD . . . . . . . . . . . 303

4.3 Beschränkung der Auswertearbeit unter Berücksichtigung der speziellen Bedürfnisse der Kanalisationstechnik . . . . . . . . . . . 304

4.4 Gang der statistischen Auswertung . . . . . . . . . . . 305

4.5 Caing der Auswertung am Beispiel der Stadt Bern . . . . . . . . 312

4.6 Die Abweichungen der theoretischen von den gemessenen Werten . . 3 I6

s. Die Ergebnisse der Auswertung . . . . . . . . . . . . . . . . . . . . 320

5.I Tabcllen der Häufigkeitsverteilung . . . . . . . . . . . . 320

5.2 Summenhäufigkeitsdiagramme . . . . . . . . . . . . . 320

5.3 Beziehung zwischen den Zentralwerten und der Regendauer . . . . 336

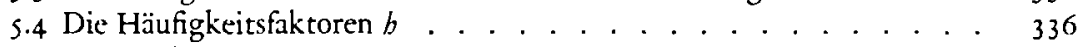

5.5 Genauigkeit der Auswertung . . . . . . . . . . . . . . 339

6. Zusammenfassung der Ergebnisse . . . . . . . . . . . . . $34 \mathbf{I}$

6.I Gleichung der Regenintensitätskurven für die praktischen Bedürfnisse ................. . . $34 \mathrm{I}$

6.2 Die Grundzahlen $G$. . . . . . . . . . . . . . . . . . . . . . 341

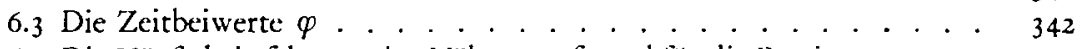

6.4 Die Häufigkeitsfaktoren $b-$ Näherungsformel für die Praxis . . . . . 344

6.5 Tabelle der Regenintensitäten für den praktischen Gebrauch . . . . 344

7. Scblussbetracbtung . . . . . . . . . . . . . . . . 348

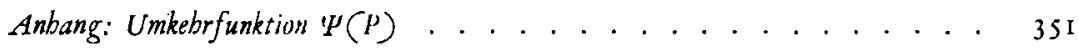

Literaturverzeicbnis . . . . . . . . . . . . . . . . 352 
Formelzeichen

\begin{tabular}{|c|c|c|}
\hline Zeichen & Einheit & Bedeutung \\
\hline$A$ & $\mathrm{~mm}$ & Konstanten in der Funktion, die die Abhängigkeit von der \\
\hline$B$ & Min. & Regendauer beschreibt. \\
\hline$C$ & & Koeffizient im vereinfachten Häufigkeitsfaktor \\
\hline$F$ & ha & Fläche des Einzugsgebietes (für Berechnungspunkt $P$ ) \\
\hline$G$ & l/s ha & $\begin{array}{l}\text { Grundzahl, d.h. Intensität eines Regens von } 15 \text { Minuten } \\
\text { Dauer, die durchschnittlich jedes Jahr einmal erreicht oder } \\
\left.\text { überschritten wird (a.o. auch } r_{15}^{(1)}\right)\end{array}$ \\
\hline$K$ & & $\begin{array}{l}\text { Von } z \text { abhängiger Wert in der Formel für den praktischen } \\
\text { Gebrauch }\end{array}$ \\
\hline$N$ & $\mathrm{~mm}$ & Niederschlagshöhe (eines Regenabschnittes) \\
\hline$P_{i}$ & $\%$ & $\begin{array}{l}\text { Summenhäufigkeit, das heisst Häufigkeit, mit welcher im } \\
\text { Beobachtungszeitraum die Intensität den Betrag } i \text { über- } \\
\text { schreitet }\end{array}$ \\
\hline$Q_{R}^{P}$ & $1 / \mathrm{s}$ & Maximaler Regenwasserabfluss im Bereclinungspunkt $P$ \\
\hline$S$ & Jahre & Dauer des Beobachtungszeitraumes \\
\hline$T$ & Min. & Regendauer (eines Regenabschnittes) \\
\hline$U_{i}$ & & $\begin{array}{l}\text { Wert der stochastischen Variablen } \imath \text { für die Intensität } i \text { aus } \\
\text { theoretischer Verteilungsfunktion }\end{array}$ \\
\hline $\begin{array}{l}a \\
b\end{array}$ & & $\left\{\begin{array}{l}\text { Konstanten in der linearisierten Zeitfunktion des Zentral- } \\
\text { wertes }\end{array}\right.$ \\
\hline$e$ & & Basis der natürlichen Logarithmen $(2,7183)$ \\
\hline$f$ & $(\%)$ & Relativer Fehler der Regenintensität (in Prozenten) \\
\hline$b$ & & Häufigkeitsfaktor \\
\hline$i$ & $\mathrm{~mm} / \mathrm{Min}$. & Regenstärke (Regenintensität) \\
\hline$k$ & & $\begin{array}{l}\text { Konstante in der linearisierten Verteilungsfunktion (im } \\
\text { Wahrscheinlichkeitsnetz) }\end{array}$ \\
\hline$m$ & & Rang von Grössen, nach absteigendem Betrag geordnet \\
\hline$n$ & & Jährliche Häufigkeit eines Ereignisses \\
\hline$n_{j}$ & & $\begin{array}{l}\text { Anzahl Fälle, die im Beobachtungszeitraum in einem Inten- } \\
\text { sitätsintervall } \Delta i \text { eingetroffen sind }\end{array}$ \\
\hline$P_{i}$ & & $\begin{array}{l}\text { Summe der Anzahlen aller Fälle grösser als der Intensitäts- } \\
\text { wert } i\end{array}$ \\
\hline$p_{s}$ & & Gesamtsumme der Anzahlen aller beobachteten Fälle \\
\hline$r$ & $1 / \mathrm{s} \mathrm{ha}$ & Regenspende (Regenintensität), auch $r_{T}^{(z)}$ \\
\hline$t$ & & $\begin{array}{l}\text { Konstante in der linearisierten Verteilungsfunktion (im } \\
\text { Wahrscheinlichkeitsnetz) }\end{array}$ \\
\hline$u$ & & Stochastische Variable in der Verteilungsfunktion \\
\hline
\end{tabular}




\begin{tabular}{|c|c|c|}
\hline Zeichen & Einheit & Bedeutung \\
\hline$u_{i}$ & \multirow{20}{*}{ Jahre } & $\begin{array}{l}\text { Wert der stochastischen Variablen } u \text { für die Intensität } i \text { aus } \\
\text { Messung }\end{array}$ \\
\hline$x$ & & Koordinaten im ebenen, rechtwinkligen System \\
\hline$y$ & & \\
\hline$z$ & & $\begin{array}{l}\text { Durchschnittliche Zeitspanne, innert welcher ein Intensitäts- } \\
\text { wert einmal erreicht oder überschritten wird }\end{array}$ \\
\hline$\Phi$ & & Gaußsche Integralfunktion (Verteilungsfunktion) \\
\hline$\Psi$ & & Umkehrfunktion der Gaußschen Integralfunktion \\
\hline$\alpha$ & & $\begin{array}{l}\text { Einheit auf der Ordinatenachse des Wahrscheinlichkeits- } \\
\text { netzes }\end{array}$ \\
\hline$\beta$ & & $\begin{array}{l}\text { Einheit auf der Abszissenachse des Wahrscheinlichkeits- } \\
\text { netzes }\end{array}$ \\
\hline$\gamma$ & & $\begin{array}{l}\text { Einheit auf der Ordinatenachse im Diagramm Zentralwert/ } \\
\text { Regendauer }\end{array}$ \\
\hline$\delta$ & & $\begin{array}{l}\text { Einheit auf der Abszissenachse im Diagramm Zentralwert/ } \\
\text { Regendauer }\end{array}$ \\
\hline$\vartheta$ & & $\begin{array}{l}\text { Steigungswinkel der Geraden im Diagramm Zentralwert/ } \\
\text { Regendauer }\end{array}$ \\
\hline$x$ & & $\begin{array}{l}\text { Steigungswinkel der Summenhäufigkeitslinien (im Wahr- } \\
\text { scheinlichkeitsnetz) }\end{array}$ \\
\hline$\lambda$ & & Köffizienten im Häufigkeitsfaktor \\
\hline$\mu$ & & \\
\hline$\xi$ & & $\begin{array}{l}\text { Zentralwert der Verteilungsfunktion (statistischer Para- } \\
\text { meter) }\end{array}$ \\
\hline$\sigma$ & & $\begin{array}{l}\text { Streuungsmass der Verteilungsfunktion (statistischer Para- } \\
\text { meter) }\end{array}$ \\
\hline$\varphi$ & & Zeitbeiwert \\
\hline$\psi$ & & Abflussbeiwert (Scheitelabflussbeiwert) \\
\hline $\mathfrak{n}$ & & $\begin{array}{l}\text { Anzahl der verwendeten Wertepaare bei der Fehlerberech- } \\
\text { nung }\end{array}$ \\
\hline $\mathbf{r}$ & & Korrelationskoeffizient \\
\hline
\end{tabular}




\section{Einleitung}

Für die Dimensionierung verschiedener Bauwerke auf dem Gebiete der Wasserwirtschaft ist die genaue Kenntnis der Niederschlagsverhältnisse von wesentlicher, wenn nicht sogar von ausschlaggebender Bedeutung. In der Kanalisationstechnik werden die Regenwasserkanäle bei einer En:wässerung im Trennsystem sowie sämtliche Kanäle des Mischsystems grösstenteils nach folgender Gleichung berechnet:

$Q_{R}^{P}=r \psi F$, wobei

$Q_{R}^{P}=$ maximaler Regenwasserabfluss im Berechnungspunkt $P$ in $1 / \mathrm{s}$,

$r=$ für die Grösstwassermenge im Punkt $P$ massgebende Regenintensität in $1 / \mathrm{s}$ ha,

$\psi=$ Abflussbeiwert (Scheitelabflussbeiwert),

$F==$ Einzugsgebiet für den Berechnungspunkt $P$ in ha.

Der für die Dimensionierung der Kanalisationen massgebende maximale Regenwasserabfluss ist somit um so einwandfreier erfassbar, je genauer die einzelnen Faktoren ermittelt werden können, die den Grösstabfluss bedingen. Dabei zeigt es sich, dass für die Dimensionierung einer Kanalisation für eine gewählte, als zweckmässig erachtete Rückstauhäufigkeit nicht der Regen mit der maximalen Regenintensität massgebend ist, sondern in der Regel ein Regren, dessen Regendauer der Abflusszeit vom obern Ende der Kanalisation bis zum Berechnungspunkt $P$ entspricht, wobei Ausnahmen, je nach der Topographie des Geländes und der Form des Einzugsgebietes zu berücksichtigen sind.

Viele Städte und Ortschaften in der Schweiz besitzen heute bereits generelle Kanalisationsprojekte, die häufig auf fragwürdigen Regrenintensitätskurven unbekannter Häufigkeit aufgebaut sind. Einerseits wurden die Regenintensitätskurven, mit wenigen Ausnahmen, nach verschiedenen Verfahren ausgewertet, die nicht miteinander verglichen werden können; anderseits werden Regenintensitätskurven, wie sie zum Beispiel für die Stadt Zürich ermittelt wurden, auf Gebiete mit ganz anderem Witterungscharakter übertragen: Dabei werden oft gefühlsmässige Zuschläge oder Abminderungen an den Zürcher Kurven vorgenommen, die dem Witterungscharakter der Gegend Rechnung tragen sollen. Hie und da werden die Zürcher Kurven unbesehen übernommen.

Um einen besseren Einblick in die Niederschlagsverhältnisse bei Starkregenfällen in der. Schweiz zu erlangen und zugleich eine Vereinheitlichung der Ergebnisse zu erzielen, hat es die Eidg. Anstalt für Wasserversorgung, Abwasserreinigung und Gewässerschutz an der ETH, Zürich 
(EAWAG), übernommen, die der Anstalt zugänglichen Regenmessstreifen nach einem einheitlichen Verfahren auszuwerten und die Ergebnisse der Praxis zur Verfügung zu stellen. Dabei wurden der EAWACs Hilfen mannigfacher Art gewährt. Auf den 1. Mai 1958 bewilligte der Schweiz. Schulrat die Einstellung einer Arbeitskraft, die sich ausschliesslich mit der Auswertung der Regenstreifen befassen konnte. Zudem gewährten uns die Kantone Bern und Baselland sowie die Gemeinden Uster und Rorschach und durch Subventionierung dieser Arbeiten die Kantone Zürich und St. Gallen, einen Beitrag an die Auswertekosten. Für die Auswertung der Regenstreifen der Stadt Basel konnte das Ingenieurbüro ED. Holinger, Liestal, gewonnen werden. Die Regenstreifen von Locarno und Como wurden durch Dipl.-Ing. A. RivA, Iocarno, ausgewertet, und zwar nach dem nämlichen Verfahren, das im folgenden beschrieben wird. Die Beschaffung der Regenstreifen stiess gliicklicherweise auf keine Schwierigkeiten. Im Gegenteil: Von sämtlichen Stellen wurden uns die gewünschten Registrierstreifen in ausserordentlich bereitwilliger Weise für die Dauer der Auswertung zur Verfügung gestellt. Unserer Behörde, den kantonalen und kommunalen Verwaltungen und den meteorologischen Anstalten, die uns durch ihre Unterstützung und ihr Entgregenkommen die Arbeit wesentlich erleichtert haben, möchten wir auch an dieser Stelle danken. Ein besonderer Dank gebührt der Schweiz. Meteorologischen Zentralanstalt in Zürich, die unsere Bestrebungen in hohem Masse unterstützt hat.

\section{Bisher in der Schweiz verwendete Regenintensitätskurven}

\section{I Stalt Zürich}

Aus der Erkenntnis heraus, dass bei grösseren Einzugsgebieten der Regenwasserabfluss nicht proportional mit der Einzugsfläche zunimmt, hat Stadtingenieur BürkLI-ZIEGLER im Jahre r 880 seine Abflussformel entwickelt, die eine universelle Verbreitung gefunden hat und die als sogenannte Verzögerungsformel in der Kanalisationsliteratur, selbst in neuester Zeit noch, erwähnt wird. Da damals noch keine Registrierungen der Niederschläge vorlagen, konnte man nicht wissen, dass die sogenannte Verzögerung durch die Abnahme der Regenintensität mit der Regendauer bei gleicher Häufigkeit bedingt ist und nicht durch einen Abminderungsbeiwert, entsprechend der vierten W'urzel des Quotienten aus Gefälle in \% und Abflussfläche in ha. Doch gebührt BüRKLI-ZIEGLER das Verdienst, als erster erkannt zu haben, dass die allgemeine Abfluss- 
gleichung einer Korrektur bedürfe, im Sinne einer Abminderung der maximalen Regenwassermenge bei grösseren Einzugsgebieten, das heisst längeren Fliesszeiten.

Auf Grund einer Auswertung von einzelnen Starkregen hat Dipl.-Ing. J. MüLl.ER, früherer Chef des Kanalisationsbüros der Stadt Zürich, Potenzkurven für die Stadt Zürich veröffentlicht, die als rojährige, 2ojährige und sojährige Regenintensitätskurven des Tiefbauamtes der Stadt Zürich (TAZ) nicht nur für die Dimensionierung der Kanalisation der Stadt Zürich verwendet wurden, sondern auch heute noch als Grundlage für die Bemessung der Kanalisation anderer Gemeinden dienen.

Erst später, in den Jahren 1936 und 1940 , sind die grundlegenden Veröffentlichungen von Prof. REINHOLD, Darmstadt, erschienen, die eine systematische Auswertung der Regenstreifen nach einem einheitlichen Verfahren bezweckten und die in ganz Deutschland Eingang gefunden hatten. Um eine Beziehung zwischen den Zürcher Regenintensitäten und denjenigen von Deutschland zu erhalten, hat Dipl.-Ing. A. KRopF, früherer Chef der Technischen Abteilung der EAWAG, Zürich, die Regenstreifen der Meteorologischen Zentralanstalt, Zürich, erneut nach dem von REINHOLD veröffentlichten Verfahren ausgewertet und dabei zum Teil wesentliche Abweichungen gegenüber den TAZ-Kurven gefunden. So entsprach zum Beispiel die sogenannte sojährige Regenintensitätskurve der Auswertung des TAZ ungefähr einem Regen, der durchschnittlich nach KROPF alle Is Jahre einmal erreicht oder überschritten wird ${ }^{1}$ ). Die Ergebnisse der Auswertung von A. KROPF sind in der Schweiz. Bauzeitung erschienen [I].

\subsection{Stadt St. Gallen}

Das Kanalisationswesen. der Stadt St. Gallen wies schon frühzeitig einen bemerkenswert hohen Stand auf. Auf Grund von Schreibregenmesseraufzeichnungen wurden seinerzeit nach einem unbekannten Verfahren zwei Regenintensitätskurven ermittelt, die alle ro bzw. alle 20 Jahre einmal erreicht oder überschritten werden. Die ersterwähnte Regenintensitätskurve wird für die Dimensionierung der Kanalisation in den Aussengebieten, die letztere für die Bemessung der Kanäle im Stadtzentrum. verwendet.

Neuere Auswertungen nach dem von REINHOLD veröffentlichten Verfahren [2] ergaben, dass die Iojährige Kurve auch mit den neueren Er-

1) Nach den neuesten, hier vorgelegten Resultaten wird allerdings diese Regenintensitätskurve durchschnittlich alle 9-12 Jahre einmal erreicht oder überschrítten werden. 
Zuscummenstellung der perwendeten Regenintensitätsgleichungen

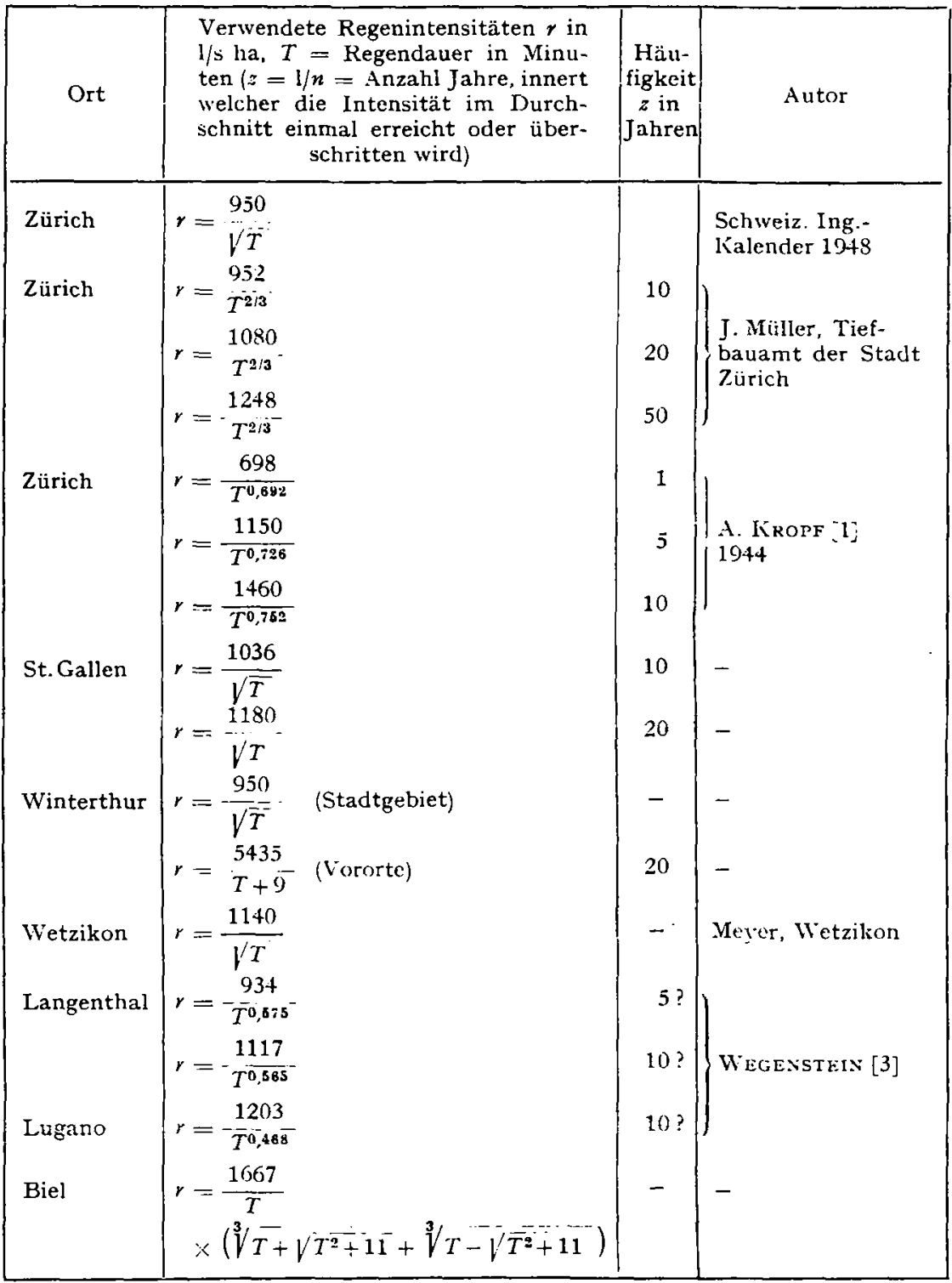

hebungen in guter Übereinstimmung steht, während die frühere 20jährige Kurve eher als zu hoch erachtet wurde. Im übrigen weist die Gleichung der Regenintensitäten von St. Gallen einen andern Aufbau auf als die 
früher verwendeten Gleichungen. Sie entspricht bereits den von REINHOLD für Deutschland gefundenen Beziehungen.

\subsection{Stalt Wintertbur}

Die Stadt Winterthur verwendet für das engere Stadtgebiet eine Potenzformel älteren Aufbaus. Für die Vororte hingegen wird eine Regenintensitätskurve angewendet, die der von Prof. ReINHOLD angegebenen Beziehung entspricht.

\subsection{Weitere Veröffentlicbungen}

Im Rahmen der Vorträge des "Einführungskurses zur Behandlung der wichtigsten Fragen der Gewässerverunreinigung und $A b$ wasserreinigung (Herbst 1936)» hat Dipl.-Ing. M. WEGENSTEIN, Zürich [3], verschiedene Regenintensitätskurven veröffentlicht, die jedoch nicht nach einem einheitlichen Verfahren ausgewertet worden sind. Auch fehlen Angaben der Häufigkeiten der Ereignisse, so dass ein Vergleich der Regenintensitätskurven untereinander unstatthaft ist. Auch diese Veröffentlichung zeigt, dass es wünschbar ist, eire einheitliche Grundlage für die Ermittlung der Regenintensitätskurven für die ganze Schweiz zu schaffen.

\section{Vorhandenes Beobachtungsmaterial}

\section{I Vorbandene Scbreibregenmesser}

Für die Auswertung der Regenmeßstreifen kommen nur Meßstellen in Frage mit einem Beobachtungszeitraum von etwa ro Jahren und darüber. Aus diesem Grunde scheiden mehrere Regenmeßstationen für die Auswertung zun vornherein aus. $\mathrm{Da}$ in verschiedenen grenznahen Gebieten keine brauchbaren Registrierungen vorliegen, wurden auch benachbarte ausländische Stationen (Bregenz und Como) bearbeitet. Soweit möglich, wurden 30 jährige Beobachtungsperioden ausgewertet, und zwar in Zeitraum von ca. I928-1957, um die gleichen Jahrzehnte für alle Messstationen zu erfassen und um unabhängig von eventuellen Änderungen in den Witterungsverhältnissen im Verlaufe grösserer Zeiträume zu sein. Dies bedingte auch eine teilweise Neubearbeitung der Regenmeßstreifen von Zürich, deren Auswertung für die Jahre 1927-1956 erfolgte. Die Auswerteergebnisse von A. KROPF der Jahre r927-I94I wurden beibehalten, jedoch die Intensitäten der Jahre I942-1956 zusätzlich berücksichtigt. Damit ergibt sich eine gewisse Abweichung der Regenintensitäten gegenüber der früheren Veröffentlichung (siehe Bemerkung im Abschnitt 2.I). 
Die bestehende Auswertung der Regenmeßstreifen von St. Gallen [2] wurde ebenfalls statistisch neu bearbeitet. Auch hier treten geringe Abweichungen gegenüber der Erstpublikation auf. Diese neuen Regenintensitäten können unmittelbar mit denjenigen der übrigen Städte verglichen werden.

Eine Zusammenstellung der Schreibregenmesser findet sich in der unter Abschnitt 3.2 aufgeführten Tabelle I sowie in der Abb. I.

\subsection{Vorbandent Regenstreifen}

Über die uns zugegangenen und ausgewerteten Regenstreifen gibt ebenfalls die Tabelle I Auskunft. Die Regenstreifen waren Tagesstreifen mit Io-Minuten-Teilung auf der Abszissenachse und to mm Regenhöhe auf der Ordinate ( $\mathrm{r} \mathrm{Std.}=\mathrm{r}, 6 \mathrm{~cm}$; $\mathrm{mm}$ Regenhöhe $=0,8 \mathrm{~cm}$ ). Streifen mit unzweckmässigerer Teilung, die in gewissen Jahren in Bregenz verwendet wurden, sind bei der Auswertung nicht berücksichtigt worden. Einzig dieStation Uster verwendete Tagesstreifen mit 20-Minuten-Teilung (I Std. $=I, 08 \mathrm{~cm}$ ) und $40 \mathrm{~mm}$ Regenhöhe (I mm Regenhöhe $=0,5 \mathrm{~cm}$ ).

Die Aufzeichnungen auf den einzelnen Streifen waren von unterschiedlicher Qualität. Abgesehen von Unterbrüchen infolge Reparaturen, Lükken in der Registrierung, Fehlanzeigen infolge mangelhaften Funktionierens der Gefässentleerung oder Versagen der Schreibvorrichtung waren die Streifen, die während mehreren Tagen verwendet wurden, oft zuwenig deutlich und eindeutig angeschrieben. Die Verwendung des nämlichen Tagesstreifens für die Aufzeichnung mehrerer Tage hat zwar den Vorteil, dass das Aktenmaterial und die Kosten für die Streifen sich wesentlich reduzieren. Dies setzt jedoch eine deutliche Beschriftung der Streifen voraus sowie die Auffüllung des Schwimmergefässes entsprechend etwa I mm Regenhöhe bei der unumgänglichen täglichen Kontrolle des Registrierapparates an Tagen ohne Regenfall. Ein Beispiel von Regenregistrierungen zeigt Abb. 2.

\section{Das Auswerteverfahren}

\section{I Verfabren von Reinbold}

\section{I I Der Begriff der Regenintensität}

Die Regenintensität kann entweder als Regenstärke $i$ in Millimetern pro Minute oder als Regenspende $r$ in Litern pro Sekunde und Hektare 

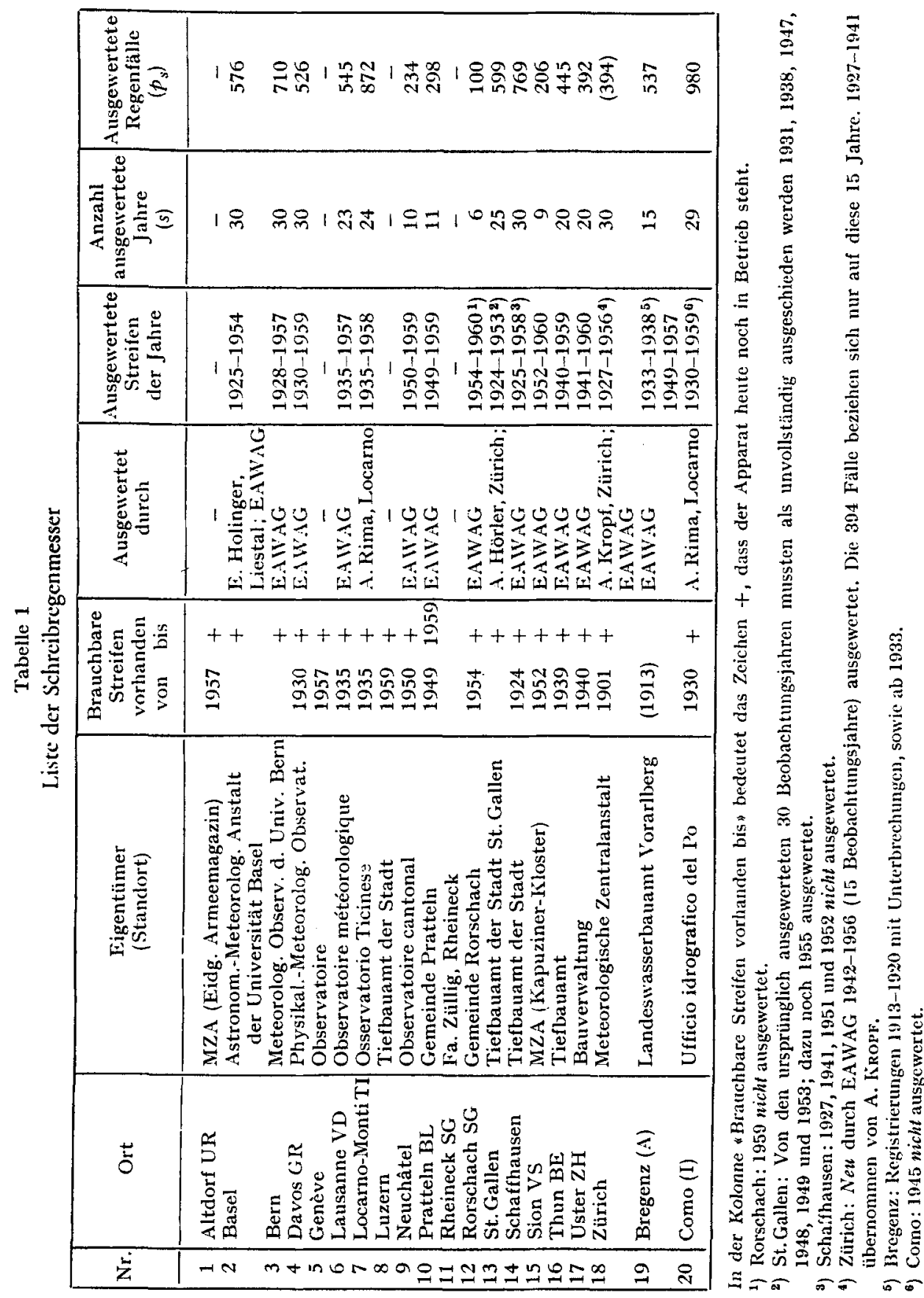


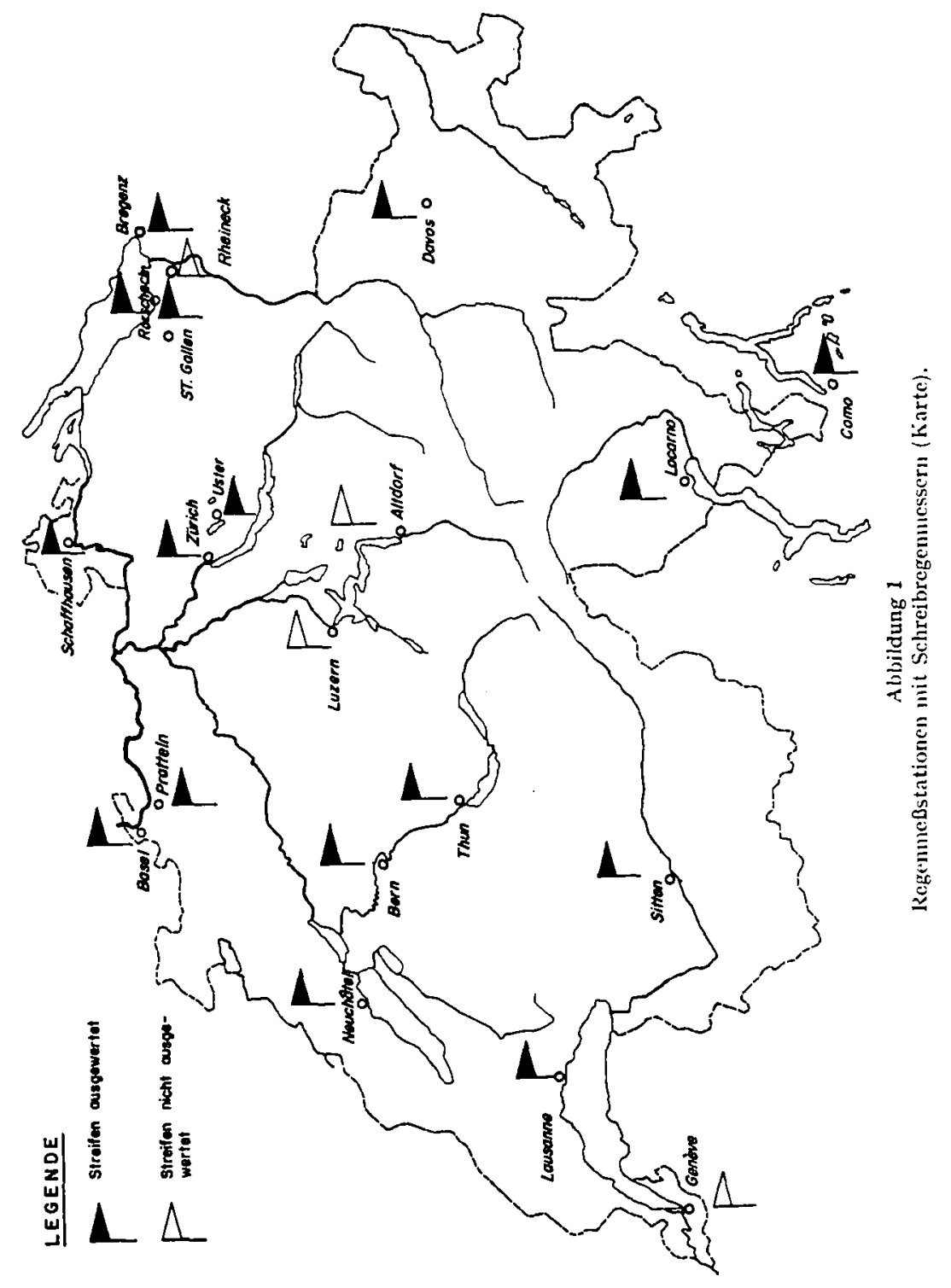




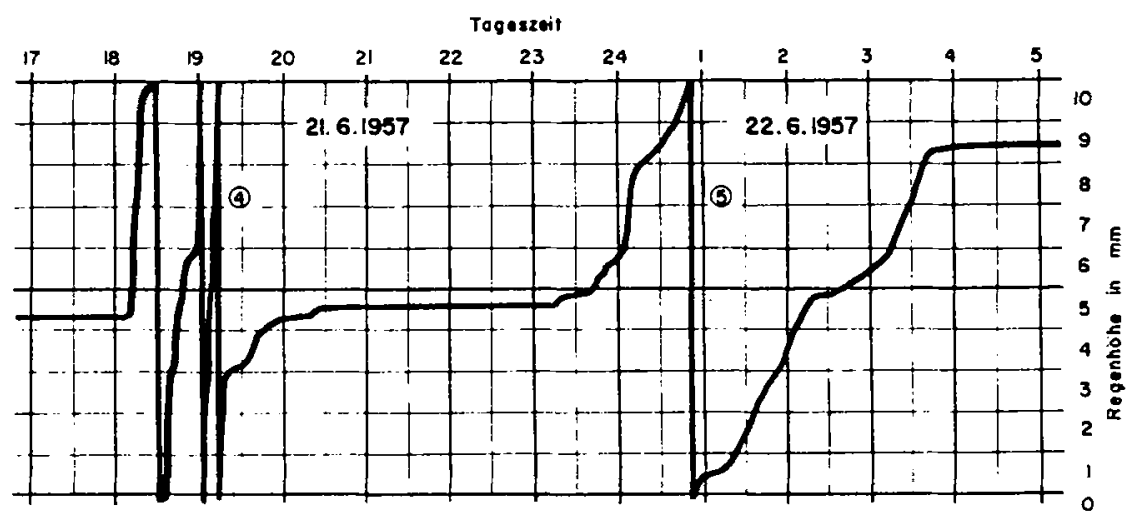

Abbildung 2

beispicle von Regenregistrierungen.

angegeben werden. Beide Werte besagen das gleiche; es handelt sich dabei nur um einen Wechsel der Masseinheiten.

Die Regenstärke $i$ wird definiert als $i=\Lambda N / \Delta T \mathrm{~mm} / \mathrm{min}$, das heisst, man bildet den Quotienten aus der in einem bestimmten Zeitabschnitt gefallenen Niederschlagshöhe in Millimetern $(J N)$ und dem Betrag desselben Zeitabschnittes in Minuten $(\Delta T)$.

Aus $\mathrm{I} \mathrm{mm} / \mathrm{min}=10000 \mathrm{l} / \mathrm{min} \mathrm{ha}=166,7 \mathrm{l} / \mathrm{s}$ ha folgt die Regenspende $r$ (in der Schweiz meist mit dem Oberbegriff «Regenintensität» bezeichnet) als

$$
r=16 C, 7 \cdot \frac{d N}{\Delta T} 1 / \mathrm{sh} \mathrm{h},
$$

wobei $\Delta N=$ Regenhöhe eines Regenabschnittes in Millimetern, $\neg T=$ Regendauer dieses Abschnittes in Minuten.

(In der Folge werden die Zeichen $\Delta$ der Einfachheit halber weggelassen).

Die jährliche Häufigkeit " gibt an, wie oft innert eines Jahres eine bestimmte Regenintensität erreicht oder überschritten wird. Anderseits gibt die Zahl $\approx$ die durchschnittliche Anzahl Jahre an, innert welcher der Intensitätswert einmal erreicht oder überschritten wird. Es gilt die Beziehung $n \approx=\mathrm{I}$.

\subsection{Gesichtspunkte für die Auswertung}

REINHOLD hat seine grundlegenden A uswerteverfahren von Schreibregenmesseraufzeichnungen in mehreren Publikationen veröffentlicht $[4,5]$.

Zur Auswertung kommen nicht sämtliche registrierten Regenfälle, sondern nur jene, die als «beachtliche Starkregen» bezeichnet werden. Die 
untere Grenze für diese beachtlichen Starkregen wurde von REINHOLD wie folgt festgelegt:

Regenhöhe $=$ I mm für eine Regendauer $T \leq$ Io $\mathrm{min}$,

Regenstärke $=0,1 \mathrm{~mm} / \mathrm{min}$ für eine Regendauer ro $<T<60 \mathrm{~min}$,

Regenhöhe $=6 \mathrm{~mm}$ für eine Regendauer $60 \leq T \leq 360 \mathrm{~min}$,

Regenstärke $=\mathrm{I} \mathrm{mm} / \mathrm{h}$ für einen Dauerregen von $T>360 \mathrm{~min}$,

Regen von weniger als I Minute Dauer wurden grundsätzlich nicht bërücksichtigt.

Jeder Regenfall, der die obenerwähnten Grenzen überschreitet, wurde tabellarisch aufgetragen, und zwar in Regendauerstufen unterteilt. Für den Teilregen von zum Beispiel 5 Minuten Dauer gilt es, innerhalb eines Regenfalles die maximale Regenhöhe für diesen Zeitabschnitt herauszulesen, ebenso für die Zeitabschnitte von Io, I5, 20,30,45,60, 90, I20, I 50, I80, 240 und 360 Minuten Regendauer. Werden sämtliche beachtlichen Starkregen so ausgewertet und tabellarisch zusammengestellt, so können daraus die Verte jeder Regendauerstufe mit der höchsten, der zweithöchsten, der dritthöchsten usw. Regenintensiẗ̈t herausgelesen und entsprechend die Häufigkeit des Regens ermittelt werden. REINHOLD gibt neben dem tabellarischen Verfahren auch ein graphisches Auswerteverfahren an, wobei jeder einzelne beachtliche Starkregen in seinen einzelnen Phasen entsprechend den Regendauerstufen in einem logarithmischen Netz aufgetragen wird. Dadurch erhält man die Regrenintensitätskurven der Einzelregen. Es ist jedoch aus dem von REINHOLD angeführten Beispiel ersichtlich, dass sich dieses Verfahren für die Praxis offenbar nur schlecht eignet, da bereits bei wenig Beobachtungsjahren ein Liniengewirr entsteht, dessen Weiterverarbeitung mühsam ist.

\subsection{Kritik am Verfahren pon Reinhold}

REINHOLD hat die einzelnen Regenfälle nur so weit ausgewertet, als beachtliche Regenintensitäten festgestellt werden konnten. So ergeben sich vereinzelte Regenfälle, die nur in den ersten Regendauerstufen aufgeführt sind, hingegen in den Kolonnen mit grösserer Regendauer nicht mehr in Erscheinung treten. Das Auswertematerial ist somit nicht homogen auf die einzelnen Regendauerstufen verteilt. Besonders auffallend ist dies bei der graphischen Auswertung, wo einzelne Regenfälle plötzlich abbrechen. Es kann somit vorkommen, dass ein extremer Starkregen von zum Beispiel 14 Minuten Regendauer für die I5. Minute nicht mehr berücksichtigt wird. Dabei hätte dieser Regen, wenn seine Regenhöhe 
durch is Minuten (und die folgenden Regendauerstufen) dividiert worden wäre, beachtliche Regenstärken ergeben, die für die Wahl der Häufigkeit hätten berücksichtigt werden sollen. Wir sind der Auffassung, dass mit dem Abklingen des Regens die anschliessenden geringen, mitunter gegen Null verlaufenden Regenintensitäten für die ganze Beobachtungsdauer mit zu berücksichtigen sind. Dadurch erhält man ein lückenloses Beobachtungsmaterial, das sich auch für die Auswertung zu statistischen Zwecken eignet. [6], [7] (siehe zum Beispiel in Tabelle 2, Regen Nr. I).

Auf die Schwierigkeit in der praktischen Anwendung der graphischen Aufzeichnung der Regenintensitäten der einzelnen Regenfälle wurde bereits hingewiesen. Es fragt sich zudem bei diesem Verfahren, ob es statthaft ist, im logarithmischen Netz geradlinig zu interpolieren und den so erhaltenen Polygonzug demjenigen der Regenmeßstreifen gleichzusetzen.

Durch die vorgehenden Bemerkungen soll der Wert der Reinholdschen Arbeit in keiner Weise geschmälert werden. Es wurde deshalb auch von uns grundsätzlich nach dem Verfahren von REINHOLD ausgewertet, unter Anwendung nachfolgend aufgeführter Abweichungen.

\subsection{Beschränkung der Auswertearbeit unter Berücksichtigung der speziellen Bedürfnisse der Kanalisationstechnik}

Bei der Auswertung der Regenstreifen der Schweiz wurde versucht, gegenüber den Vorschlägen von REINHOLD zeitliche Einsparungen in der Auswertearbeit zu erzielen, um das gesteckte Ziel innert nützlicher Frist erreichen zu können. Da es uns vor allem darauf ankam, Unterlagen für kanalisationstechnische Zwecke zu erhalten, konnte die Auswertearbeit ohne Qualitätseinbusse durch folgende Massnahmen eingeschränkt werden.

\subsection{Untere Auswertegrenze der Regen}

Anstelle der von REINHOLD vorgeschlagenen Abstufungen der untern Auswertegrenze wurden in der Regel nur Regen untersucht, die - wenn auch nur für kurze Zeit - Intensitäten von $0,2 \mathrm{~mm}$ pro Minute und darüber aufwiesen. Als einzige Ausnahme wurde für die Auswertung der Streifen von Sitten diese untere Grenze auf $0, \mathrm{I} \mathrm{mm} / \mathrm{min}$ festgesetzt. Wurde ein Regen als würdig für die Auswertung befunden, so wurde er für sämtliche Regendauerstufen ausgewertet. Somit sind von solchen Regenfällen auch diejenigen Regenteile beachtet worden, die geringere Intensitäten als $0,2 \mathrm{~mm}$ pro Minute aufwiesen. 


\subsection{Regendauerstufen}

Die einzelnen Zeitabschnitte der zu untersuchenden Regendauerstufen wurden starr gewählt, ganz unabhängig davon, wie der Verlauf der registrierten Summenkurve der Regenhöhen sich gestaltete. Zudem wurde Wert darauf gelegt, gleiche Zeitintervalle zu berücksichtigen, und zwar Regendauerwerte von IO, 20, 30, 40, 50 und 60 Minuten. Zusätzlich sind die Regenintensitäten von 5 und IS Minuten Regendauer ausgewertet worden (nicht aber für Uster, als Folge der Blatteilung). Für die Auswertung der Meßstreifen von Como ergab sich wegen der dort vorliegenden Wochenregistrierungen eine andere Wahl der Regendauerstufen. Es wurden ausgewertet Regen von I5, 20, 25, 30, 35, 40, 45, 50, 55 und 60 Minuten Dauer. Regen von uiber 60 Minuten Dauer blieben bewusst unberücksichtigt. Da die Laufzeit in den Kanalisationen vom obersten Ende eines Kanalisationsstranges bis zum nächsten Regenauslass oder bis zur Kläranlage, selbst bei den grossen Schweizer Städten, kaum 30 Minuten überschreiten wird, erwies sich die Auswertung von Regen über 60 Minuten Dauer als nicht notwendig. Da die Meßstreifen von St. Gallen nicht mehr neu ausgewertet wurden (siehe auch Abschnitt 3.I), sind für diese Station nur die bereits vorhandenen Daten der Regendauerstufen von 10, 20, 30 und 60 Minuten bearbeitet worden.

\subsection{Tabellarische Auswertungen}

Bevorzugt wurde die tabellarische Auswertung der Regenstreifen. Da sich die zeichnerische Auswertung als zeitraubender und für die weitere statistische Auswertung als wenig zweckmässig erwies, ist bewusst darauf verzichtet worden. Nur die Resultate der tabellarischen Auswertungen wurden zur Veranschaulichung in sogenannten Staffeldiagrammen (Histogramme), Abb. 3, graphisch dargestellt. Für die Auswertung gelangte Tabelle 2 zur Anwendung.

\subsection{Gang der statistiscben Auswertung}

Bei den den Verfassern aus der Literatur bekannten Auswertungen der Regenstreifen wurde so vorgegangen, dass für alle Regendauerstufen die Intensitätswerte mit der gleichen Häufigkeit herausgesucht wurden, um vorerst die Abhängigkeit der Intensität von der Regendauer zu bestimmen. Hierbei stellte man für jede gewählte Häufigkeit eine spezielle Gleichung für die Intensität als Funktion der Regendauer in der Weise 


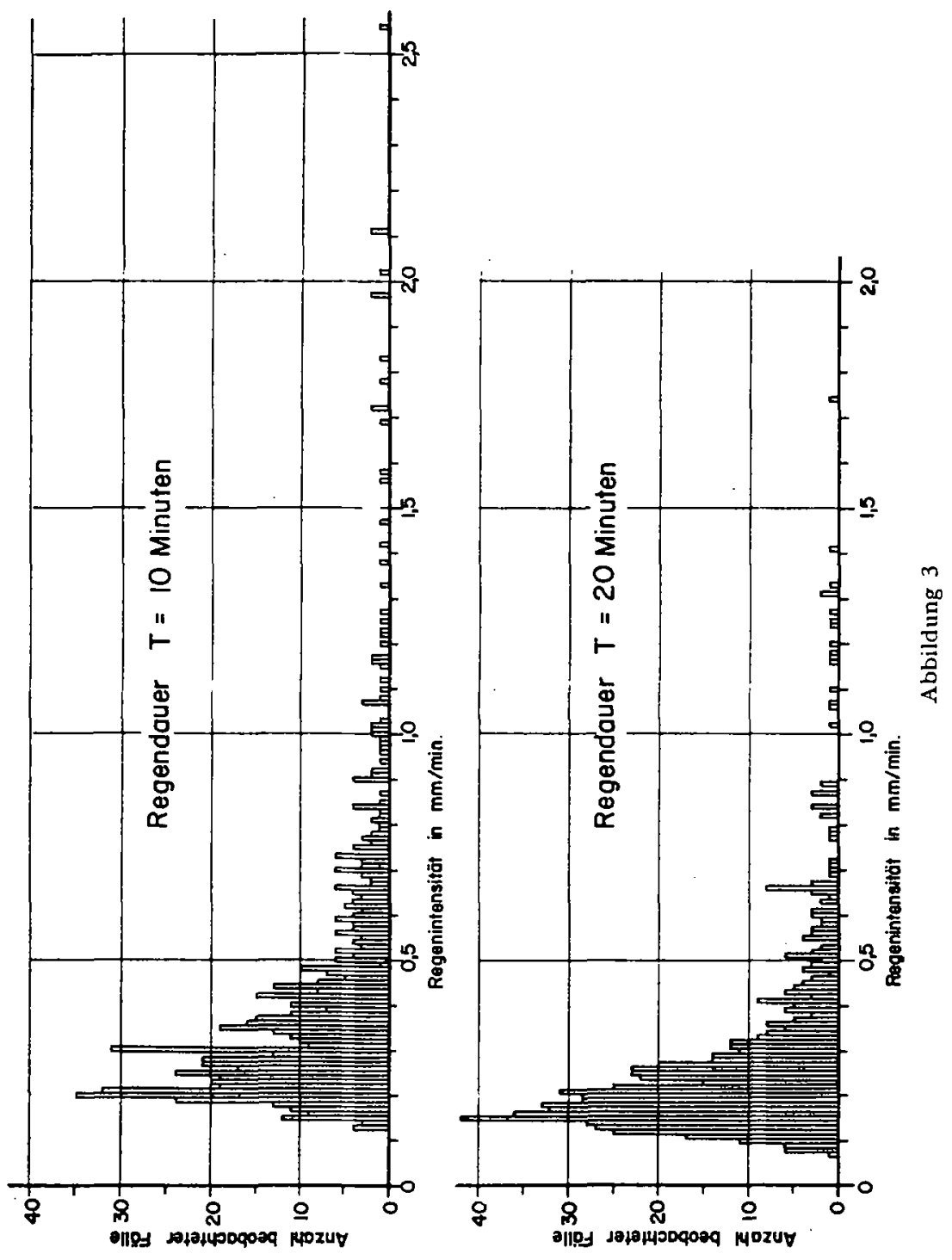




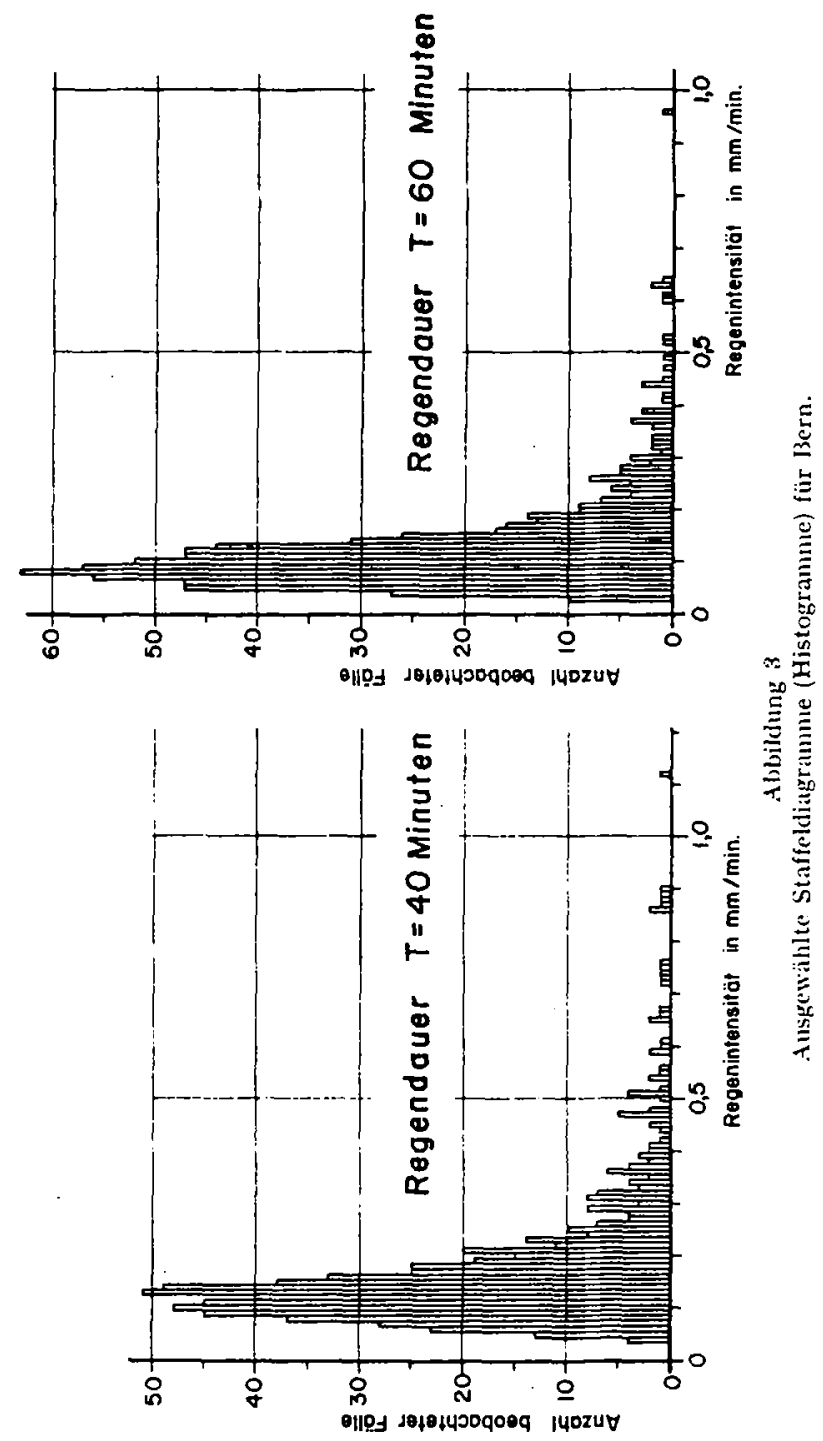


Tabelle 2

Regenmeßstation: Bern

Jahr: 1957, Auswertegrenze: $0,2 \mathrm{~mm} / \mathrm{min}$, Blatt $\mathrm{Nr}$. I

\begin{tabular}{|c|c|c|c|c|c|c|c|c|c|c|c|c|c|}
\hline \multirow[b]{2}{*}{ Nr. } & \multirow[b]{2}{*}{ Datum } & \multicolumn{3}{|c|}{$5 \min$} & \multicolumn{3}{|c|}{$10 \mathrm{~min}$} & \multicolumn{3}{|c|}{$15 \mathrm{~min}$} & \multicolumn{3}{|c|}{$20 \mathrm{~min}$} \\
\hline & & 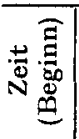 & $\begin{array}{l}\Delta N \\
\mathrm{~mm}\end{array}$ & $-\frac{E}{E}$ & 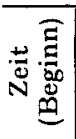 & $\begin{array}{l}\Delta N \\
\mathrm{~mm}\end{array}$ & $-\frac{\vec{E}}{E}$ & 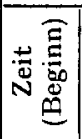 & $\begin{array}{l}\Delta N \\
\mathrm{~mm}\end{array}$ & $\rightarrow \frac{\mathrm{g}}{\mathrm{E}}$ & 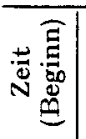 & $\begin{array}{l}\Delta N \\
\mathrm{~mm}\end{array}$ & $\infty \frac{E}{E}$ \\
\hline 1 & 12. 5. & 16.25 & 6.1 & 1,22 & 16.25 & 8,4 & 0,84 & 16.25 & 8,5 & 0,57 & 16.25 & 8,6 & 0.43 \\
\hline 2 & 16. 5. & 6.25 & 3,6 & 0,72 & 06.20 & 4,8 & 0,48 & 06.20 & 5,2 & 0,35 & 06.20 & 5,6 & 0,28 \\
\hline 3 & 30.5 . & 16.30 & 5,2 & 1,04 & 16.25 & 9,6 & 0,96 & 16.25 & 11,9 & 0,79 & 16.25 & 13,2 & 0,66 \\
\hline 4 & & 19.08 & 5,6 & 1,12 & 19.03 & 9,2 & 0,92 & 18.58 & 13,7 & 0,91 & 18.58 & 17,4 & 0,87 \\
\hline 5 & $21 . / 22.6$. & 00.00 & 1,3 & 0,26 & 00.00 & 2,1 & 0,21 & 23.55 & 2,3 & 0,15 & 23.50 & 2,6 & 0,13 \\
\hline 6 & 23.6 & 03.05 & 1,6 & 0,32 & 3.05 & 1,9 & 19 & 03.05 & 2,3 & & 03.05 & 2,6 & 0,13 \\
\hline 7 & 24.6. & 38 & 4,0 & 0,80 & 11.38 & 4,8 & 0,48 & 11.38 & 5,3 & 0,35 & 11.38 & 5,8 & 0,29 \\
\hline 8 & 8.7. & & 1,8 & 0,36 & 17.48 & 3,4 & & 17.43 & 4,6 & 0,31 & 17.43 & 5,2 & 0,26 \\
\hline 9 & 8./9.7. & 00.05 & 3,0 & 0,60 & 00.00 & 4,8 & 0,48 & $00.00 \mid$ & 6,4 & 0,43 & 23.55 & 7,2 & 0,36 \\
\hline
\end{tabular}

\begin{tabular}{|c|c|c|c|c|c|c|c|c|c|c|c|c|c|}
\hline \multirow[b]{2}{*}{$\mathrm{Nr}$. } & \multirow[b]{2}{*}{ Datum } & \multicolumn{3}{|c|}{$30 \mathrm{~min}$} & \multicolumn{3}{|c|}{$40 \mathrm{~min}$} & \multicolumn{3}{|c|}{$50 \mathrm{~min}$} & \multicolumn{3}{|c|}{$60 \mathrm{~min}$} \\
\hline & & 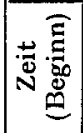 & $\begin{array}{l}\Delta N \\
\mathrm{~mm}\end{array}$ & 帕 & 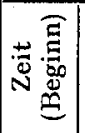 & $\begin{array}{l}\Delta N \\
\mathrm{~mm}\end{array}$ & . $\frac{\vec{E}}{\underline{E}}$ & 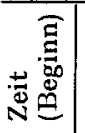 & $\begin{array}{l}\Delta N \\
\mathrm{~mm}\end{array}$ & $\cdot \frac{\stackrel{\Xi}{\Xi}}{\underline{\Xi}}$ & 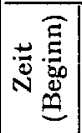 & $\begin{array}{l}\Delta N \\
\mathrm{~mm}\end{array}$ & م $\frac{\stackrel{\Xi}{\Xi}}{\Xi}$ \\
\hline 1 & 12. 5. & 16.25 & 8,6 & 0,29 & 16.25 & 8.6 & 0,21 & 16.25 & 8,6 & 0,17 & $\mid 16.25$ & 8.6 & 0,14 \\
\hline 2 & 16. 5 . & 06.20 & 7,8 & 0,26 & 06.20 & 8,3 & 0,21 & 06.20 & 8,8 & 0,18 & 06.10 & 9,3 & 0,16 \\
\hline 3 & 30.5. & 16.25 & 13,9 & 0,46 & 16.25 & 14,6 & 0,36 & 16.25 & 15,0 & 0,30 & 16.25 & 15,3 & 0,26 \\
\hline 4 & & 18.48 & 18,9 & 0,63 & 18.38 & 20,4 & 0,51 & 18.28 & 21,9 & 0,44 & 18.18 & 23,5 & 0,3 \\
\hline 5 & $21 . / 22.6$. & 23.40 & 3,0 & 0,10 & 23.40 & 3,3 & 0,08 & 23.40 & 3,6 & 0,07 & 23.40 & 4.2 & 0,07 \\
\hline 6 & 23.6 & 03.05 & 2,7 & 0,09 & 02.55 & 2,7 & 0,07 & 02.55 & 2,7 & 0,06 & 02.55 & & 0,05 \\
\hline 7 & 24.6 & 11.38 & 6,2 & 0,21 & 11.38 & 6,4 & 0,16 & 11.38 & 6,8 & 0,14 & 11.38 & 7,1 & 0,12 \\
\hline 8 & 8.7. & & 5,4 & 0,18 & 17.43 & 5,5 & 0,14 & 17.43 & 5,9 & 0,12 & 17.43 & 6,7 & 0,11 \\
\hline 9 & $8 . / 9.7$. & 23.45 & 7,6 & 0,25 & 23.45 & 7,7 & 0,19 & $23.45 \mid$ & 7,7 & 0,15 & 23.45 & 7,7 & 0,13 \\
\hline
\end{tabular}

Die Regen Nr. 4 und 5 sind in Abb. 2 dargestellt, wie sie auf den Pluviogrammen aufgezeichnet waren.

auf, dass sie sich möglichst den ermittelten Intensitätswerten anglich. Dabei wurden die Werte ebenfalls in einem Staffeldiagramm (Histogramm), und zwar für jede Regendauerstufe, getrennt, aufgetragen und durch $\mathrm{Ab}$ zählen vom grössten ermittelten Wert aus nach rückwärts die Werte gleicher Häufigkeit ermittelt. - Wenn man mit $m$ den Rang des Intensitätswertes bezeichnet, also $m=\mathrm{I}$ für den grössten Wert, $m=2$ für den zweitgrössten usw., bestimmt sich die jährliche Häufigkeit zu $n=m / S$, wenn $S$ die Dauer der Beobachtungsperiode in Jahren angibt. Bei einer 
30jährigen Beobachtungsperiode wurde zum Beispiel der drittgrösste Wert $(m=3)$ herausgegriffen und dieser somit mit der Häufigkeit $n=\mathrm{I} / \mathrm{IO}$, bzw. $z=$ Io bezeichnet. Der dreissigste Wert $(m=30)$ liefert dann den sog. einjährigen Regen, da $n=z=\mathrm{I}$. Es stellt sich nun die Frage, ob wirklich bei allen Regendauerstufen diese Werte auch die bezeichneten Häufigkeiten aufweisen. Da im Bereich der extremen Starkregenfälle nur wenig Messwerte vorhanden sind, so sind auch die zu erwartenden Streuungen offensichtlich sehr gross. Es scheint darum kaum gerechtfertigt, solche durch Abzählen gewonnene Werte als Regenintensitäten mit einer bestimmten Häufigkeit zu bezeichnen, da es sich dabei um zufällige Werte handelt, die stark von der gewählten Auswerteperiode abhängen.

Es wurde deshalb versucht, einen anderen Weg einzuschlagen, also nicht direkt die rohen Auswertedaten durch eine Gleichung darzustellen, sondern zuerst das wahrscheinlichkeitstheoretische Gesetz $\mathrm{zu}$ ermitteln, welches dem zufälligen Eintreffen der Starkregenfälle zugrunde liegt. Auf diese Weise wurden alle durch die Auswertung der Registrierstreifen gewonnenen Daten, nicht nur die extremsten Werte, für die Ermittlung der Beziehung zwischen Regenintensität, Regendauer und Häufigkeit erfasst. Damit kann dem einen Hauptgesetz der mathematischen Statistik, dem Gesetz der grossen Zahl, Rechnung getragen werden ${ }^{1}$ ). Dadurch werden wahrscheinlichere Werte für die Regenintensitäten bestimmter Häufigkeit erhalten. Auch ist dann eine Extrapolation in gewissen Grenzen eher zulässig. Auch in der amerikanischen Fachliteratur wird eine solche Behandlung dieses und ähnlicher Probleme empfohlen [8].

Betrachtet man die Staffeldiagramme (Abb. 3), so fällt auf, dass die Anzahlen der Regenfälle, nach Intensitätsstufen geordnet, nicht irgendwelche Werte annehmen, sondern dass sie offensichtlich gesetzmässig über den Intensitätsbereich verteilt sind. Ebenso sieht man sofort, dass es sich um eine schiefe Häufigkeitsverteilung handelt. $\mathrm{Zu}$ einem ähnlichen Ergebnis gelangte auch JOHANNSEN [9] bei der Aufstellung von Dauerkurven der Regenintensitäten. Gesetze, die eine schiefe Häufigkeitsverteilung bestimmen, gibt es mehrere. Es galt deshalb durch Versuche herauszufinden, welches Gesetz in unserm Fall die tatsächliche Verteilung am besten wiedergibt. Als die zuerst versuchte Poissonsche

\footnotetext{
1) Dieses Gesetz besagt, dass die gemessene Häufigkeit eines Ereignisses mit einer bestimmten Wahrscheinlichkeit des Eintreffens sich erst um beliebig wenig von dieser Wahrscheinlichkeit unterscheidet, wenn die Anzahl der beobachteten Fälle genügend gross wird.
} 
Verteilung als nicht zutreffend erkannt werden musste, blieb unter anderem die Möglichkeit, durch geeignete Transformation der normalen Gaußschen Häufigkeitsverteilung das Ziel zu erreichen. Die Transformation wird so vorgenommen, indem nicht die Grösse selbst - in unserm Falle die Regenintensität - normal verteilt ist, sondern. eine Funktion dieser Grösse. Die Frage ist nur, welche Funktion im Falle der Regenintensitäten zweckmässig ist. Bei der Genauigkeit des vorliegenden Rohmaterials genügt eine Verteilungsfunktion, die durch zwei statistische Parameter bestimmt ist, vollauf [6], [ro]; ebenfalls reicht es aus, wenn diese Parameter auf graphischem Wege ermittelt werden (vgl. auch Abschnitt 4.6). Diese zwei Parameter sind bei der Normalverteilung schon als Mittelwert (bzw. Zentralwert) und Streuung gegeben. Die Transformation soll also keine weitern Parameter mehr liefern. Anderseits sind die Regenintensitäten nach unten begrenzt, sie können auf keinen Fall negativ werden. Nach oben besteht keine logische Grenze. Man kann sich denken, dass jeder einmal erreichte Wert noch überschritten werden kann. Bei der Normalverteilung hingegen kann die Variable Werte von minus Unendlich bis plus Unendlich durchlaufen. Die Transformation soll also die Regenintensität $i$, die von Null bis plus Unendlich variieren kann, in eine Grösse umwandeln, die von $-\infty$ bis $+\infty$ läuft; ausserdem soll kein unbekannter Parameter darin enthalten sein. Es ist naheliegend, als Grösse, die normal verteilt werden soll, den Logarithmus der Regenintensität zu nehmen. Diese Transformation wird übrigens auch für andere Probleme häufig angewendet, so dass die erhaltene schiefe Verteilung als "logarithmische Normalverteilung» bezeichnet und in den Lehrbüchern der mathematischen Statistik [II], [I2], [I3] beschrieben wird. Im Handel sind auch entsprechende Spezialpapiere (Wahrscheinlichkeitsnetz) erhältlich. (AERNI-LEUCH, Nr. 563 von uns verwendet).

Nachdem das Prinzip gezeigt wurde, wie das umfangreiche Material, der Regenstreifen-Auswertung zweckmässig statistisch zu verarbeiten ist, bleibt noch zu erläutern, wie damit praktisch vorgegangen werden kann. Das Ziel ist $\mathrm{ja}$, einen formelmässigen Zusammenhang zwischen den Regenintensitäten und der Regendauer einerseits sowie der jährlichen Häufigkeit anderseits herzuleiten. Zuerst bildet man Intensitätsstufen mit Intervallen von $\Delta i=0, \mathrm{I} \mathrm{mm} / \mathrm{min}$, also Stufen von $0,0 \mathrm{I}-0, \mathrm{IO} ; 0, \mathrm{II}-$ 0,$20 ; 0,2 \mathrm{I}-0,30 \mathrm{~mm} / \mathrm{min}$; etc. Für jede dieser Stufen wird die Anzahl der in der Beobachtungsperiode aufgetretenen Fälle bestimmt. (Tabellen 3 , Kolonne $n_{j}$ ). Hernach berechnet man für jede Stufe die Summen- 
häufigkeit $P_{i}$ in $\%$. Die Summenhäufigkeit ist diejenige Häufigkeit, mit der in der beobachteten Zeitspanne die Intensität einen bestimmten Betrag $i$ überschreitet. Man hat also den Quotienten aus der Summe aller Fälle grösser als der Betrag $i\left(=p_{i}\right)$ und die Gesamtsumme aller beobachteten Fälle $\left(=p_{s}\right)$ zu bilden.

$$
p_{i}=\sum_{i}^{\infty} n_{j}, \quad p_{s}=\sum_{0}^{\infty} n_{j} \rightarrow P_{i} \%=\frac{p_{i}}{p_{s}} \cdot 100 \% \text {. }
$$

Die Werte von $p_{i}$ und $P_{i} \%$ sind ebenfalls in den Tabellen 3 enthalten.

Aus der Definition der «logarithmischen Normalverteilung» ergibt sich.

$$
\begin{aligned}
& P_{i}=\Phi(u)=\frac{1}{\sqrt{2 \pi}} \int_{-\infty}^{u} e^{-(1 / 2) x^{2}} d x, \quad u=\frac{\ln i-\ln \xi}{\sigma} . \\
& \left.u=\Psi\left(P_{i}\right)^{1}\right) .
\end{aligned}
$$

Durch Auflösen nach $i$ folgt:

$$
\begin{gathered}
\frac{\ln i-\ln \xi}{\sigma}=\Psi\left(P_{i}\right) \rightarrow \ln i=\sigma \cdot \Psi\left(P_{i}\right)+\ln \xi, \\
i=\xi \cdot e^{\sigma \cdot \Psi\left(P_{i}\right)} .
\end{gathered}
$$

Im folgenden wird diese Gleichung näher erläutert. Vorerst soll der Zusammenhang zwischen der Summenhäufigkeit und der jährlichen Häufigkeit $n$, bzw. ihrem reziproken Wert $z$, aufgezeigt werden. Man betrachte einen Zeitraum von $S$ Jahren. Soll ein bestimmter Intensitätswert durchschnittlich alle Jahre einmal eintreffen, so müssen in diesem Beobachtungszeitraum $S$ solche Fälle eintreffen. Soll allgemein der Intensitätswert mit einem durchschnittlichen Intervall von $z$ Jahren eintreffen, so können wir erwarten, dass er im Beobachtungszeitraum sovielmal eintritt, als das Intervall $\approx$ Jahre im Beobachtungszeitraum $S$ Jahre enthalten ist, also $p_{i}=S / z$, somit

$$
P_{i}=\frac{p_{i}}{p_{s}}=\frac{\lambda}{z}, \quad \lambda=\frac{S}{p_{s}},
$$

$\frac{1}{\lambda}=\frac{p_{s}}{S}=$ durchschnittliche Anzahl Regenfälle, die pro Beobachtungsjahr untersucht wurden.

Die beiden Werte $\sigma$ und $\xi$ als statistische Parameter sind nun noch von der Regendauer abhängig, da die Verteilung ja für jede Regendauer-

1) Eine Tabelle der Funktion $\Psi(P)$ ist in [13] enthalten. Für den hier geforderten Zweck ist jene aber zuwenig genau. Es wurde deshalb von uns eine Tafel aufgestellt (siehe Anhang), die den Bedürfnissen angepasst ist. Die Berechnung erfolgte mit Hilfe der Probit-Tabellen in [12] und $[14]$. 
stufe getrennt untersucht werden muss. Es hat sich aber gezeigt, dass der Wert $\sigma$ für eine bestimmte Station für jede Regendauer als gleichbleibend vorausgesetzt werden darf. Diese Konstanz des Wertes $\sigma$ ist nun nicht irgendeine gesuchte Hypothese, sondern liegt in der Natur der Auswertung begründet. Es werden ja für alle Regendauerstufen die gleichen Regenfälle untersucht. Das Grundereignis ist der Regen von 60 Minuten Dauer, alle andern Regendauerstufen sind nach der Definition der Auswertung in diesen 60 Minuten enthalten (Einzig Sion bestätigte diese Annahme nicht, dort variiert auch der $\sigma$-Wert mit der Regendauer. Weiteres siehe Abschnitt 5.2.).

Es ist also nur noch der Zentralwert $\xi$ ( $50 \%$-Wert) in Abhängigkeit von der Regendauer variabel. Der Zusammenhang wurde empirisch durch Korrelation gefunden. Beim Auftragen der Reziprokwerte I/ $\xi$ in Funktion der Regendauer $T$ stellte sich heraus, dass diese Werte praktisch auf einer Geraden liegen. Es ergibt sich deshalb für die Funktion von $\xi$ folgende Form:

$$
\frac{1}{\xi}=a T+b \rightarrow \xi=\frac{1}{a T+b}=\frac{A}{T+B}, \text { wenn } A=\frac{1}{a}, \quad B=\frac{b}{a} .
$$

Diese gefundenen Beziehungen in Gl. 2 eingesetzt, ergeben die Grundgleicbung für die Regenintensität:

$$
i(T, \approx)=\frac{A}{T+B} \cdot e^{\sigma \cdot \Psi(\lambda / 2)}
$$

Wir erhalten eine relativ einfache Form der Gleichung, da sich die Regenintensität, die eine Funktion der beiden Variablen Regendauer $T$ und jährliche Häufigkeit $n$ bzw. $z$ ist, als Produkt von zwei Funktionen mit nur je einer Variablen schreiben lässt. Diese Form ist allerdings für den Gebrauch in der Praxis noch nicht geeignet. Die Grundgleichung soll deshalb noch entsprechend umgeformt werden, wie im 6. Kapitel erläutert wird.

\subsection{Gang der Auswertung am Beispiel der Stadt Bern}

Um die Ausführungen des vorstehenden Abschnittes besser verständlich zu machen, soll der Gang der statistischen Auswertung am Beispiel einer RegenmeBstation ausführlicher gezeigt werden. Wir verwenden dabei die Werte der Station in Bern. Gewisse Wiederholungen von Ausführungen, die in Abschnitt 4.4 enthalten sind, lassen sich nicht vermeiden. 
Zuerst ermitteln wir die Summenhäufigkeiten für alle acht Regendauerstufen, wie in 4.4 erläutert wurde (Tab. 3.2). Diese Summenhäufigkeiten werden nun in ein sog. "Wahrscheinlichkeitsnetz» eingetragen (Abb. 4). Es ist dies ein Diagrammpapier, dessen Abszisse nach dem Gaußschen Integral, die Ordinate logarithmisch geteilt ist. Werden die aufgetragenen Punkte gleicher Regendauer miteinander verbunden, so zeigt sich, dass sie angenähert auf einer Geraden liegen, um erst bei den selteneren Werten stärker zu streuen. Auf diese Weise ist es möglich, wenigstens im Bereich, der uns interessiert, den wahrscheinlichsten Verlauf der Summenhäufigkeitslinien zu finden und damit die Parameter der Verteilungsfunktionen zu bestimmen. Zeichnet man alle 8 Summenhäufigkeitslinien (für $5-60$ Minuten Regendauer) in das gleiche Netz ein, so ergibt sich, dass die Linienzüge unter sich parallel sind. Es wird sich weiter unten zeigen, dass damit auch unsere Voraussetzung von der Konstanz des $\sigma$-Wertes erfïllt ist. Wir stellen die Summenhäufigkeitslinien als Geraden dar mit der Gleichung

$$
y=k x+t,
$$

wobei $k$ die Steigung der parallelen Geraden bedeutet (für alle Summenhäufigkeitslinien gleich gross, das heisst von der Regendauer unabhängig) und $t$ den Abschnitt auf der Ordinate, der von der Regendauer $T$ abhängt. Nach Anlage des Koordinatennetzes ist

$$
y=\alpha \ln i, \quad x=\beta \cdot \Psi\left(P_{i}\right),
$$

wobei $\alpha$ und $\beta$ die Einheiten auf Ordinaten- und Abszissenachse sind. Man setze ausserdem $t=\alpha \ln \xi$.

Mit Gl. 4 ergibt sich:

$$
\begin{aligned}
\alpha \cdot \ln i & =k \beta \Psi\left(P_{i}\right)+\alpha \ln \xi, \\
\ln i & =k \frac{\beta}{\alpha} \Psi\left(P_{i}\right)+\ln \xi .
\end{aligned}
$$

Vergleichen wir Gl. 5 mit Gl. I, folgt:

$\sigma=k \beta / \alpha ; \beta / \alpha=$ Maßstabfaktor, der aus dem Diagramm bestimmt werden kann. In unserem Fall ist $\beta / \alpha=-0,896$,

$k=\operatorname{tg} x$, kann der Zeichnung entnommen werden. Wir erhalten: $k=0,82$.

Damit ist der Parameter $\sigma$ zahlenmässig bestimmt zu $\sigma=0,82 \cdot(-0,896)$ $=-0,735$.

Als nächster Schritt werden die vorerst geschätzten Zentralwerte untersucht. Wie schon im vorhergehenden Abschnitt bemerkt, werden die reziproken Zahlen der Zentralwerte als Ordinate und die Regendauer als Abszisse aufgetragen (Abb. S). 


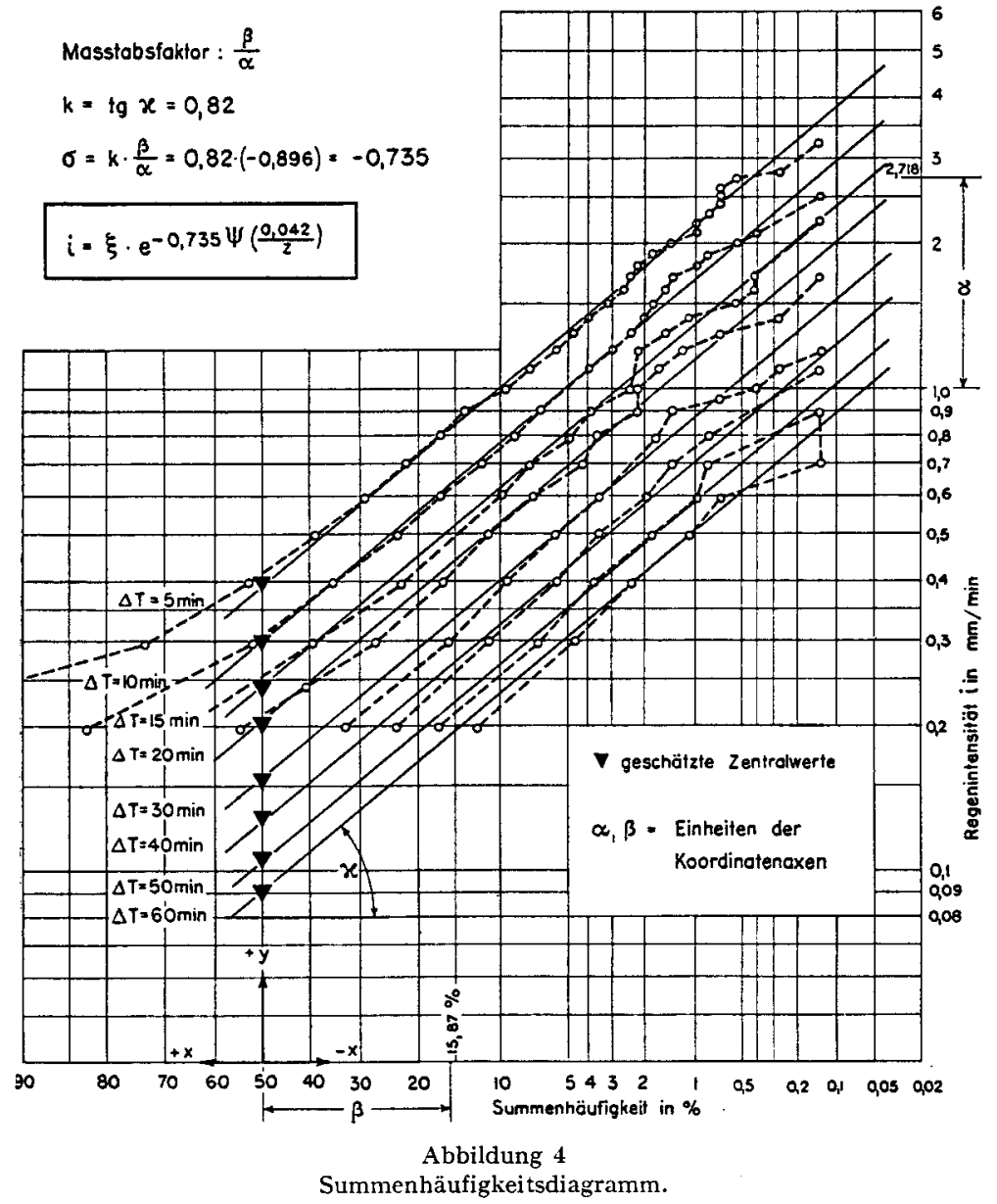

Wir erhalten eine Gerade mit der Gleichung

$$
y=a x+b, \text { wobei } y=\frac{1}{\xi} \gamma \text { und } x=T \delta .
$$

Die Grössen $\gamma$ und $\delta$ sind die Einheiten auf den Koordinatenachsen. (Maßstabfaktor $\delta / \gamma=0,2) \mathrm{a}=\operatorname{tg} \vartheta=0,744$ - kann aus der Zeichnung entnommen werden. Ebenso bestimmt man $b$ und erhält $\mathrm{I}, 785$. Wie schon früher gezeigt, führt dies zu einer Gleichung von der Form

$$
\xi=\frac{A}{T+B},
$$

wobei, mit Berücksichtigung der Maßstäbe auf den Koordinatenachsen, 


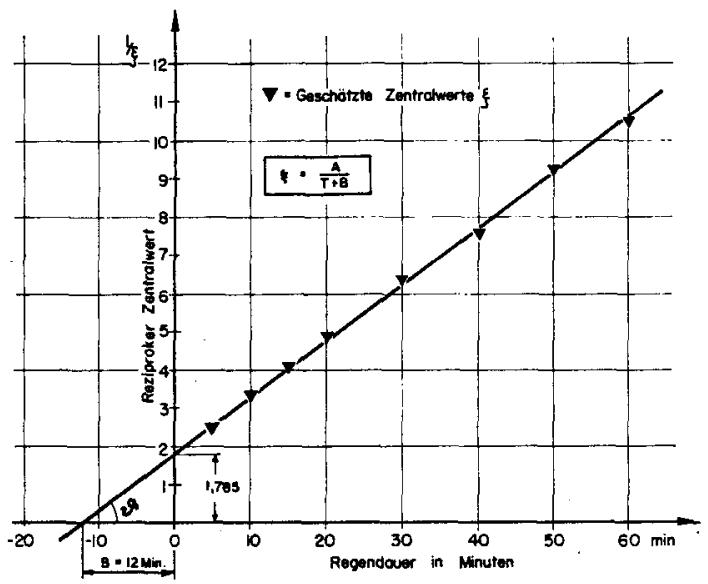

Abbildung 5

Zusammenhang zwischen Zentralwert und Regendauer.

$$
\begin{aligned}
& A=\frac{1}{a \delta / \gamma}=\frac{1}{0,744 \cdot 0,2}=6,72, \\
& B=\frac{b}{a \delta / \gamma}=\frac{1,785}{0,744 \cdot 0,2}=12,0 .
\end{aligned}
$$

Damit sind alle gesuchten Konstanten in der Grundgleichung Gl. 3 bis auf $\lambda$ gefunden. Wir hatten früher abgeleitet, dass $\lambda=S / p_{s}$ ist. Die Beobachtungsdauer $S$ betrug 30 Jahre, insgesamt wurden 710 Regenfälle untersucht. Das ergibt für $\lambda=30 / 710=0,042$. Wir können nun die Grundgleichung für die Regenintensität, wie sie die Regenmeßstation in Bern ergeben hat, anschreiben:

$$
i(T, \approx)=\frac{6,72}{T+12} \cdot e^{-0,735 \cdot \Psi(0,042 / z)} .
$$

Daraus lassen sich Regenintensitäten mit beliebiger Regendauer (sofern $5<T<60 \mathrm{~min}$ ) und beliebiger Häufigkeit bestimmen.

Als Beispiel rechnen wir die Intensität eines Regens, der Is Minuten dauert und durchschnittlich jedes Jahr einmal erreicht oder überschritten wird, das heisst, die sogenannte Grundzahl G. Wir setzen in die Gleichung $T=\mathrm{I} 5 \mathrm{~min}$ und $z=\mathrm{I}$ Jahr ein:

$$
i(\mathrm{I} 5, \mathrm{I})=\frac{6,72}{15+12} e^{-0,735 \cdot \Psi(0,042)} .
$$

Den Funktionswert von $\Psi$ entnehmen wir der Tabelle im Anhang zu

$$
\Psi(0,042)=-\mathrm{r}, 728, \quad i(\mathrm{I} S, \mathrm{I})=\frac{6,72}{27} e^{1,270}=0,886 \mathrm{~mm} / \mathrm{min} .
$$


Umgerechnet in die gebräuchlichere Dimension 1/s ha ergibt sich $r(\mathrm{I} 5, \mathrm{I})=\mathrm{I} 48 \mathrm{l} / \mathrm{s} \mathrm{ha}$.

Für die andern Stationen wurde bei der Auswertung nach dem gleichen Schema vorgegangen. Die Resultate sind im nächsten Kapitel beschrieben.

\subsection{Die Abweichung der theoretischen von den gemessenen Werten}

Bei jeder Auswertung, in der einzelne gemessene Werte durch eine algebraische Formel dargestellt werden, stellt sich die Frage nach ihrer Genauigkeit. Es soll ein Mass angegeben werden, das die Abweichungen der durch die Formel theoretisch errechneten Werte von den tatsächlich gemessenen abzuschätzen erlaubt. Im hier vorliegenden Fall ist diese Aufgabe nicht einfach zu lösen. Im Prinzip sind ja drei aufeinanderfolgende Ausgleichungen der Messwerte erfolgt, die zudem graphisch ausgeführt wurden. Erstens suchte man im Summenhäufigkeitsdiagramm für jede Regendauerstufe eine Gerade (die sogenannte «Regressionsgerade» bei analytischer Auswertung), zweitens wurden diese Geraden durch solche mit gleicher mittlerer Steigung ersetzt und drittens wurden noch die Zentralwerte (bzw. deren Reziprokwert) durch eine Gerade ausgeglichen. Alle diese Ausgleichungen überlagern sich gegenseitig.

Ein Verfahren, die Abweichungen zu erfassen, bestünde darin, zu den in Tabelle 3 vorliegenden Summenhäufigkeiten die entsprechenden Werte für die Regenintensitäten nach der Formel zu berechnen und die Differenzen zu bestimmen. Damit würden schliesslich mittlere absolute Fehler der Regenintensitäten gewonnen. Zweckmässiger wäre jedoch, wenn der mittlere relative Fehler angegeben werden könnte. Das lässt sich dadurch erreichen, dass für feste Regenintensitätswerte die gemessenen Summenhäufigkeiten, bzw. ihre Funktionen $\Psi$, mit den zugehörigen theoretisch berechneten verglichen werden. Am besten ermittelt man die mittlere quadratische Abweichung $s^{2}$ nach der bekannten Formel:

$$
s^{2}=\frac{1}{n-1} \sum_{n}\left(u_{i}-U_{i}\right)^{2} .
$$

Es bedeutet dabei $\mathfrak{n}=$ Anzahl der verglichenen Wertepaare $u_{i}$ und $U_{i}$

$$
\begin{gathered}
u_{i}=\Psi\left(P_{i} \text { gemessen }\right), \\
U_{i}=\Psi\left(P_{i} \text { theoretisch }\right) .
\end{gathered}
$$

Nach der Definition in Abschnitt 4.4 gilt 
wobei

$$
U_{i}=\frac{1}{\sigma}(\ln i-\ln \xi)
$$

$$
\xi=\frac{A}{T+B} .
$$

$U_{i}$ lässt sich also für eine gegebene Regenintensität berechnen.

Um aus der mittleren Abweichung $s$ der Summenhäufigkeiten, bzw. ihrer Funktionen $\Psi$, den mittleren relativen Fehler der Regenintensitäten zu erhalten, führe man eine Grösse $i *$ ein. Es ist dies die Regenintensität, die sich ergibt, wenn der Wert $u=\Psi\left(P_{i}\right)$ um seine mittlere Abweichung $s$ schwankt. Man kann schreiben: Bei $i=\xi e^{\left.\sigma \Psi_{i}\right)}$, wird $i^{*}=\xi e^{\sigma\left[\Psi\left(P_{i}\right) \pm s\right]}$.

Der mittlere relative Fehler $f$ der Regenintensität ist durch folgenden Ansatz bestimmt:

$$
f=\frac{i^{*}-i}{i}=\frac{i^{*}}{i}-1
$$

Die obenstehenden Ausdrücke eingesetzt, folgt daraus

$$
f=\frac{\xi \cdot e^{\sigma \cdot \Psi\left(P_{i}\right)} \cdot e^{\underline{\perp} \sigma \cdot s}}{\xi \cdot e^{\sigma \cdot \Psi\left(P_{i}\right)}}-1=e^{ \pm \sigma \cdot s}-1 \text {. }
$$

Nun kann eine Näherung angewendet werden. Da immer $s \ll 1$, gilt

$$
e^{ \pm \sigma s} \approx 1 \pm \sigma s . \quad \text { Somit wird } f \approx \pm \sigma s .
$$

Der relative Fehler der Regenintensität lässt sich also aus den mittleren Abweichungen $s$ der gemessenen von den theoretischen Summenhäufigkeiten für die einzelnen Intensitätsintervalle durch Multiplikation mit der Streuung $\sigma$ der Verteilungsfunktion direkt berechnen.

Nachfolgend sei die numerische Berechnung anhand eines Beispiels gezeigt. Es werden die Daten der Station Bern für die Regendauer $T=5$ Minuten verwendet. Die Werte für die gemessenen Summenhäufigkeiten $\left(P_{i}\right.$ gemessen) liegen in Tab. $\mathbf{3 . 2}$ vor. Die Funktionswerte $\Psi$ entnimmt man der Tabelle im Anhang.

Die theoretischen Summenhäufigkeiten, bzw. die Werte $U_{i}$ werden wie oben beschrieben berechnet, da ja $\sigma$ und $\xi$ aus der Auswertung bekannt sind $(\sigma=-0,735 ; \xi=6,72 / 5+\mathrm{I} 2=0,3953)$. 


\begin{tabular}{|c|c|c|c|c|c|}
\hline$i$ & $\begin{array}{c}P_{i} \% \\
\text { gemessen }\end{array}$ & $u_{i}$ & $U_{i}$ & $\left.\Delta^{1}\right)$ & $\Delta^{2}$ \\
\hline 0,5 & 39,6 & $-0,263$ & $-0,320$ & $-0,057$ & 0,003249 \\
\hline 0,6 & 29,4 & $-0,541$ & $-0,568$ & $-0,027$ & 0,000729 \\
\hline 0,7 & 22,5 & $-0,756$ & $-0,778$ & $-0,022$ & 0,000484 \\
\hline 0,8 & 17,3 & $-0,942$ & $-0,959$ & $-0,017$ & 0,000289 \\
\hline 0,9 & 14,0 & $-1,080$ & $-1,120$ & $-0,040$ & 0,001600 \\
\hline 1,0 & 9,74 & $-1,296$ & $-1,263$ & $+0,033$ & 0,001089 \\
\hline 1,1 & 7,60 & $-1,433$ & $-1,393$ & $+0,040$ & 0,001600 \\
\hline 1,2 & 5,77 & $-1,574$ & $-1,511$ & $+0,063$ & 0,003969 \\
\hline 1,3 & 4,80 & $-1,665$ & $-1,620$ & $+0,045$ & 0,002025 \\
\hline 1,4 & 4,08 & $-1,741$ & $-1,721$ & $+0,020$ & 0,000400 \\
\hline 1,5 & 3,24 & $-1,846$ & $-1,815$ & $+0,031$ & 0,000961 \\
\hline 1,6 & 2,68 & $-1,930$ & $-1,902$ & $+0,028$ & 0,000784 \\
\hline 1,7 & 2,54 & $-1,953$ & $-1,985$ & $-0,032$ & 0,001024 \\
\hline 1,8 & 2,26 & $-2,002$ & $-2,063$ & $-0,061$ & 0,003721 \\
\hline 1,9 & 1,83 & $-2,090$ & $-2,136$ & $-0,046$ & 0,002116 \\
\hline 2,0 & 1,41 & $-2,195$ & $-2,206$ & $-0,011$ & 0,000121 \\
\hline 2,1 & 0,99 & $-2,330$ & $-2,272$ & $+0,058$ & 0,003364 \\
\hline 2,2 & 0,99 & $-2,330$ & $-2,336$ & $-0,006$ & 0,000036 \\
\hline 2,3 & 0,85 & $-2,387$ & $-2,396$ & $-0,009$ & 0,000081 \\
\hline 2,4 & 0,70 & $-2,457$ & $-2,454$ & $+0,003$ & 0,000009 \\
\hline 2,5 & 0,70 & $-2,457$ & $-2,510$ & $-0,053$ & 0,002809 \\
\hline 2,6 & 0,70 & $-2,457$ & $-2,563$ & $-0,106$ & 0,011236 \\
\hline 2,7 & 0,56 & $-2,536$ & $-2,614$ & $-0,078$ & 0,006084 \\
\hline 2,8 & 0,28 & $-2,770$ & $-2,664$ & $+0,106$ & 0,011236 \\
\hline 3,2 & 0,14 & $-2,989$ & $-2,846$ & $+0,143$ & 0,020449 \\
\hline \multicolumn{4}{|c|}{$\mathrm{n}=25$} & $+0,570$ & 0,079465 \\
\hline \multicolumn{4}{|c|}{$s^{2}=\underline{0,079465}=0,00331104$} & & \\
\hline \multicolumn{4}{|c|}{$s=0,05754$} & $+0,005$ & \\
\hline
\end{tabular}

$f_{r e l}=\sigma s=0,04229$

1) Als Abkürzung für $u_{i}-U_{i}$ ist das Zeichen $\Delta$ gesetzt.

Für die übrigen Regendauerstufen der Station Bern sollen nur noch die wichtigsten Werte angegeben werden:

\begin{tabular}{|l|l|l|l|l|l|l|l|}
\hline $\begin{array}{c}\text { Regen- } \\
\text { dauer } T\end{array}$ & 10 Min. & 15 Min. & 20 Min. & 30 Min. & 40 Min. & 50 Min. & 60 Min. \\
\hline$\xi$ & 0,3054 & 0,2489 & 0,2100 & 0,1600 & 0,1292 & 0,1084 & 0,0933 \\
& 21 & 16 & 14 & 11 & 8 & 7 & 7 \\
$\Sigma \Delta$ & $+0,008$ & $+0,310$ & $+0,199$ & $+0,602$ & $+0,002$ & $+0,160$ & $+0,251$ \\
$\Sigma \Delta^{2}$ & 0,135784 & 0,077954 & 0,126027 & 0,163718 & 0,045126 & 0,053272 & 0,092935 \\
$s^{\mathbf{2}}$ & 0,006789 & 0,005197 & 0,009694 & 0,016372 & 0,006447 & 0,008879 & 0,015489 \\
$f=\sigma s$ & 0,06056 & 0,05299 & 0,07237 & 0,09408 & 0,05901 & 0,06926 & 0,09143 \\
\hline
\end{tabular}


Der mittlere relative Gesamtfebler $f_{g}$ der Auswertung für eine Regenmeßstation berechnet sich aus der mittleren quadratischen Gesamtabweichung $s_{g}^{2}$. Diese erhält man, indem die Summe aller Abweichungsquadrate durch die um neun ${ }^{1}$ ) verminderte Summe der Anzahl aller verwendeten Werte dividiert wird. Die weitere Rechnung ist analog der oben gezeigten. Der mittlere relative Gesamtfehler ist also wicht gleich dem arithmetischen Mittel der mittleren relativen Fehler der einzelnen Regendauerstufen.

In unserem Beispiel von Bern ist die Summe aller $\Delta^{2}$ gleich 0,774 28I und die Summe aller $\mathfrak{n}$ gleich rog. Damit wird

$$
s_{g}^{2}=\frac{0,774281}{100}=0,007743, \quad f_{g}=s_{g} \sigma=0,06467 .
$$

Im Abschnitt 4.4 wurde schon gesagt, dass die graphische Ermittlung der Parameter $\sigma$ und $\xi$ ausreichend sei. Um eine Kontrolle zu haben, wurde für Bern auch eine analytische Methode angewandt. Wir beschränken uns darauf, sie nur kurz zu skizzieren.

Die strenge Berechnung von Zentralwert und Streuung der Verteilungsfunktion führt im vorliegenden Fall zu falschen Resultaten, da die gemessene Verteilung im Bereich der kleinen Intensitäten erheblich von der theoretischen abweicht, wie auch aus den Abbildungen 3 und 4 hervorgeht. Da die kleinen Intensitäten für den hier geforderten Zweck belanglos sind, können für die Werte grösserer Intensitäten die acht Regressionsgeraden zwischen Regenintensität und Summenhäufigkeit bestimmt werden. Hernach ist der Mittelwert der Regressionskoeffizienten zu berechnen (Geraden parallel) und damit neue korrigierte Zentralwerte. Die Reziprokwerte dieser korrigierten Zentralwerte lassen sich nun wieder durch eine Regressionsgerade in Funktion der Regendauer darstellen. Schliesslich sind die Summenhäufigkeitslinien noch den ausgeglichenen Zentralwerten anzupassen. Es wurden schliesslich um weniges verschiedene Werte als bei der graphischen Methode erhalten; eine Durchrechnung der Fehlerabweichungen ergab jedoch, dass sie keineswegs besser und genauer sind als jene. Die graphische Methode ist also auch für unsere Verhältnisse durchaus genügend. Wir beschränken uns darauf, als Resultat der analytischen Auswertung die Korrelationskoeffizienten der verschiedenen Regressionsgeraden anzugeben (für die Theorie sei auf [II] verwiesen).

2) Die Division durch $n-9$ erklärt sich in diesem Fall daraus, dass durch die Bestimmung der Parameter ( 8 Zentralwerte und die Streuung $\sigma$ ) neun Freiheitsgrade wegfallen. 


\begin{tabular}{|c|c|c|c|c|c|c|c|c|c|}
\hline \multicolumn{10}{|c|}{ Korrelationskoeffizient $\mathfrak{r}$ für die Regressionsgeraden } \\
\hline \multicolumn{9}{|c|}{ Zwischen Summenhäufigkeiten und Regenintensitäten } & \multirow{2}{*}{$\begin{array}{c}\text { Zwischen } \\
\text { Zentralwerten } \\
\text { und Regen- } \\
\text { dauer }\end{array}$} \\
\hline $\begin{array}{l}\text { Regen- } \\
\text { dauer } T\end{array}$ & $5 \mathrm{Min}$. & 10Min. & $15 \mathrm{Min}$. & 20Min. & 30Min & $40 \mathrm{Min}$ & 50 Min. & $60 \mathrm{Min}$. & \\
\hline $\mathfrak{r}$ & 0,9968 & 0,9924 & 0,9928 & 0,9742 & 0,9883 & 0,9834 & 40,9834 & 0,9865 & 0,9996 \\
\hline
\end{tabular}

\section{Die Ergebnisse der Auswertung}

\subsection{Tabellen der Häufigkeitsperteilung}

Da die Tabellen der Häufigkeitsverteilung die Grundlage für jede weitere Verarbeitung der Daten bilden, sollen diese für die von der EAWAG ausgewerteten Stationen zusammengestellt werden. Dadurch ist es später möglich, die erhaltenen Werte auch für andere Auswertezwecke oder andere Auswertemethoden zu benützen, sofern dies wünschbar sein sollte. Die Häufigkeitsverteilung der Regenintensitäten für die einzelnen Meßstationen geht aus den Tabellen 3.I-3.I5 hervor. Eine weitere Erläuterung dürfte sich erübrigen, nachdem am Beispiel der Stadt Bern die Methode der Aufstellung solcher tabellarischer Zusammenstellungen ausführlich beschrieben wurde.

\subsection{Summenbäufigkeitsdiagramme}

Wir nehmen davon Abstand, diese Summenhäufigkeitsdiagramme für alle ausgewerteten Stationen in Form von Abbildungen wiederzugeben. An Hand der Tabellen über die Häufigkeitsverteilung ist es ohne weiteres möglich, diese zu rekonstruieren. Die Gleichung der Summenhäufigkeitslinien sind nach Abschnitt 4.4 von der Form

$$
\ln i=\sigma \Psi\left(\frac{\lambda}{z}\right)+\ln \xi,
$$

wobei für die einzelnen Meßstationen die in der Tab. 4 aufgeführten Werte für die verschiedenen Konstanten erhalten wurden. Die Grösse der $\xi$-Werte ist im Abschnitt 5.3 aufgeführt.

Einzig für Sion war es nicht gelungen, die oben angegebene Beziehung zu finden, da das Material für die ausgewertete relativ kurze Beobachtungsperiode grosse Inhomogenitäten aufwies. Der Wert $\sigma$ ändert mit der Regendauer. Würde man aber auch die Abhängigkeit von $\sigma$ 


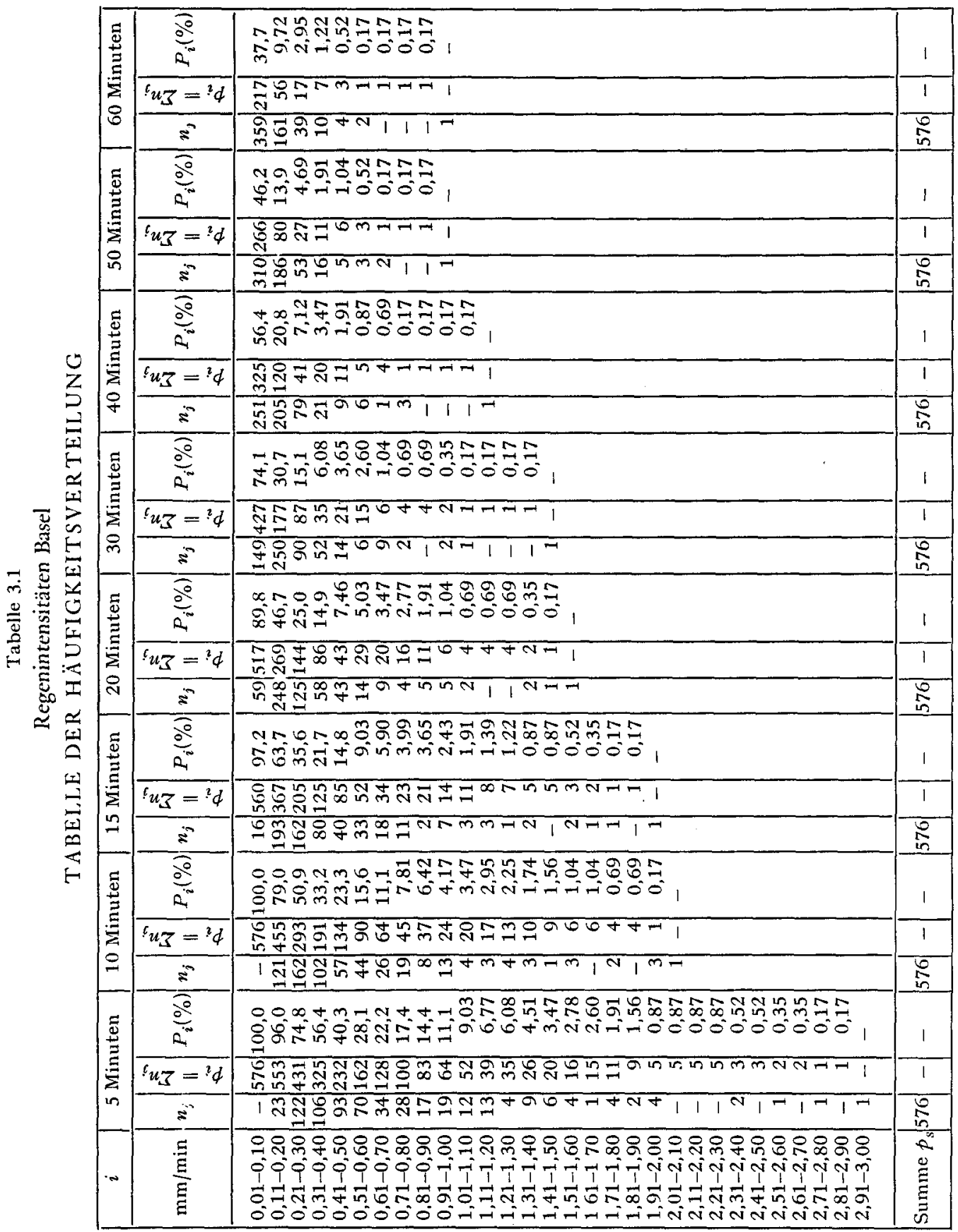




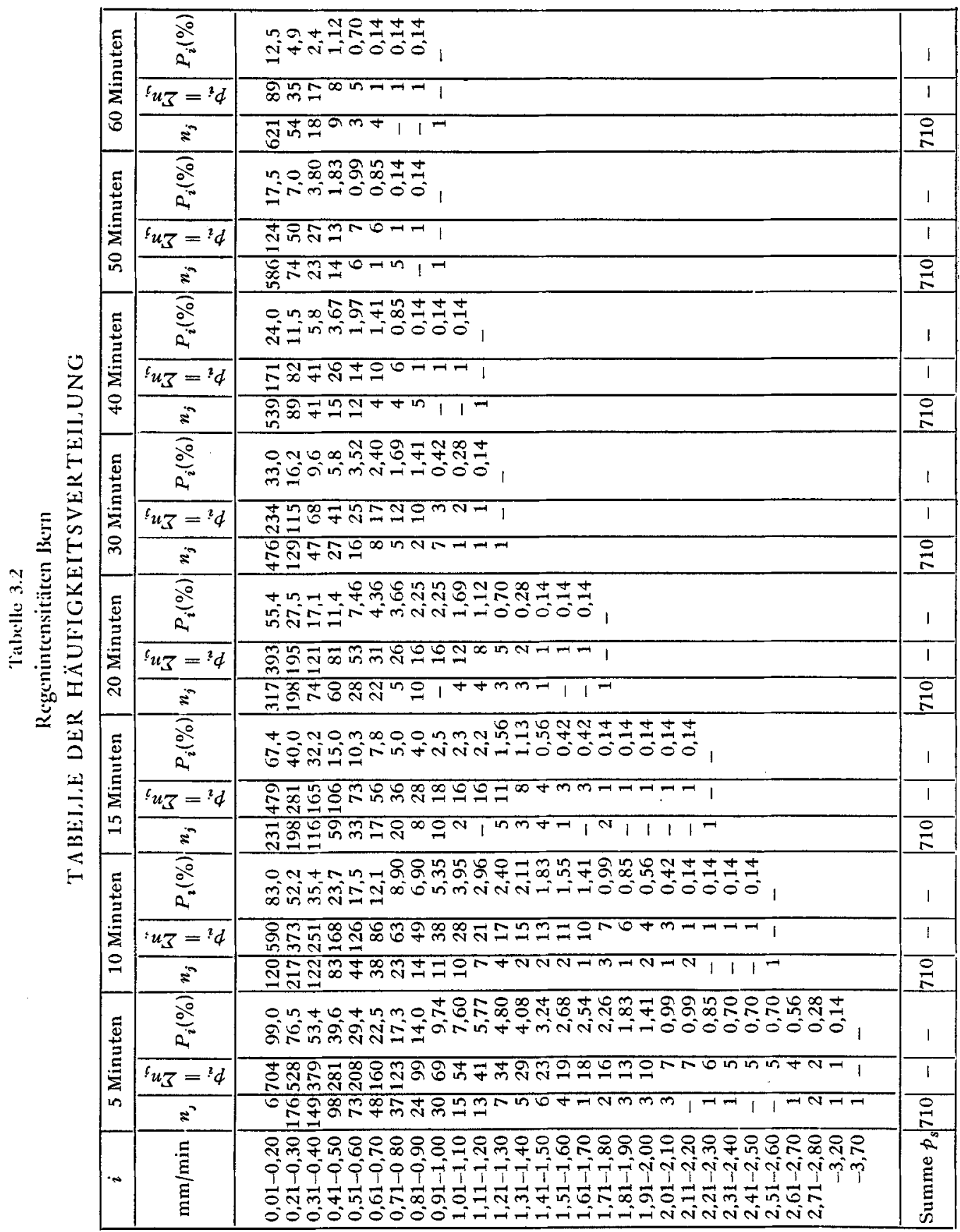




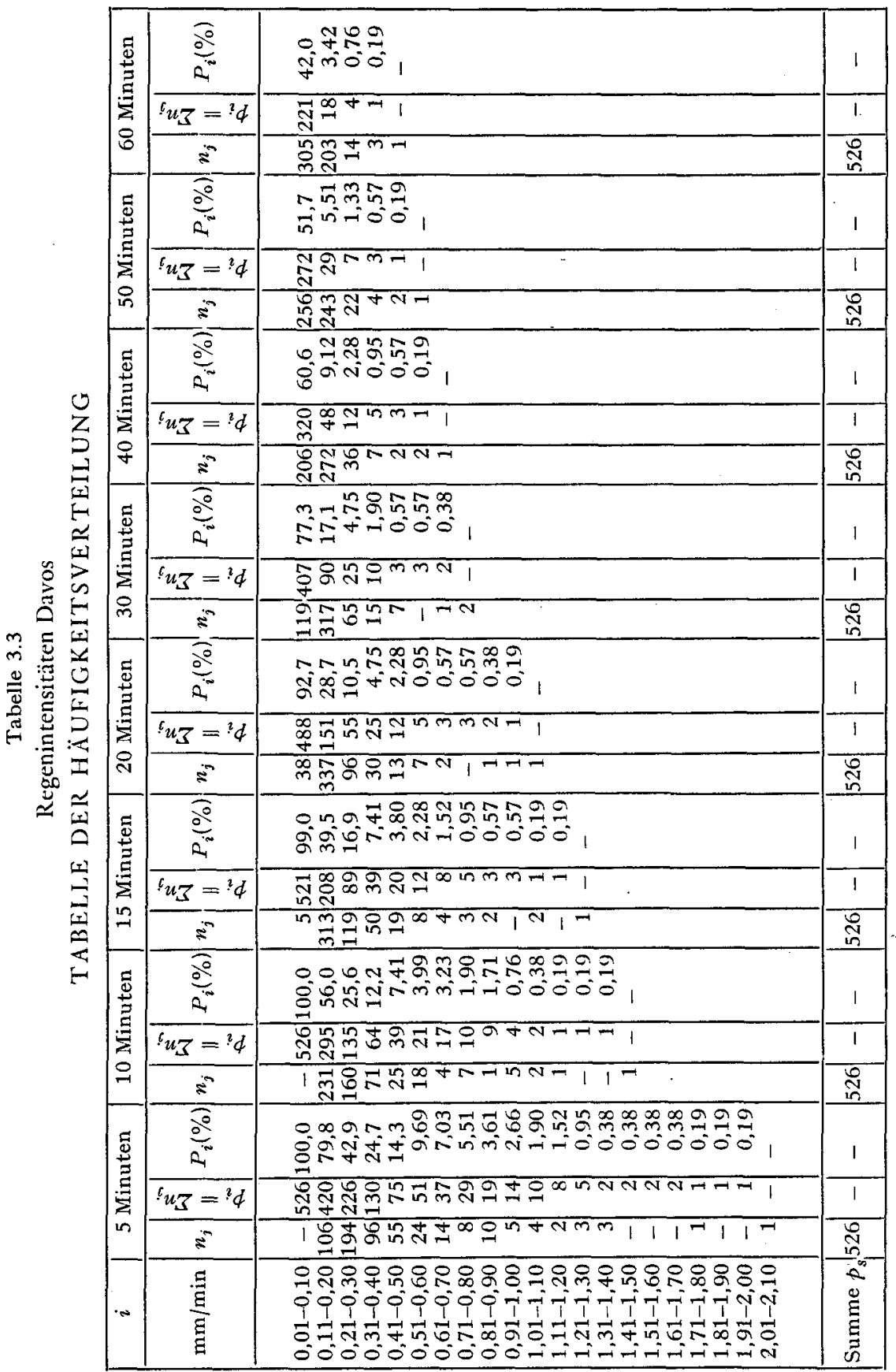




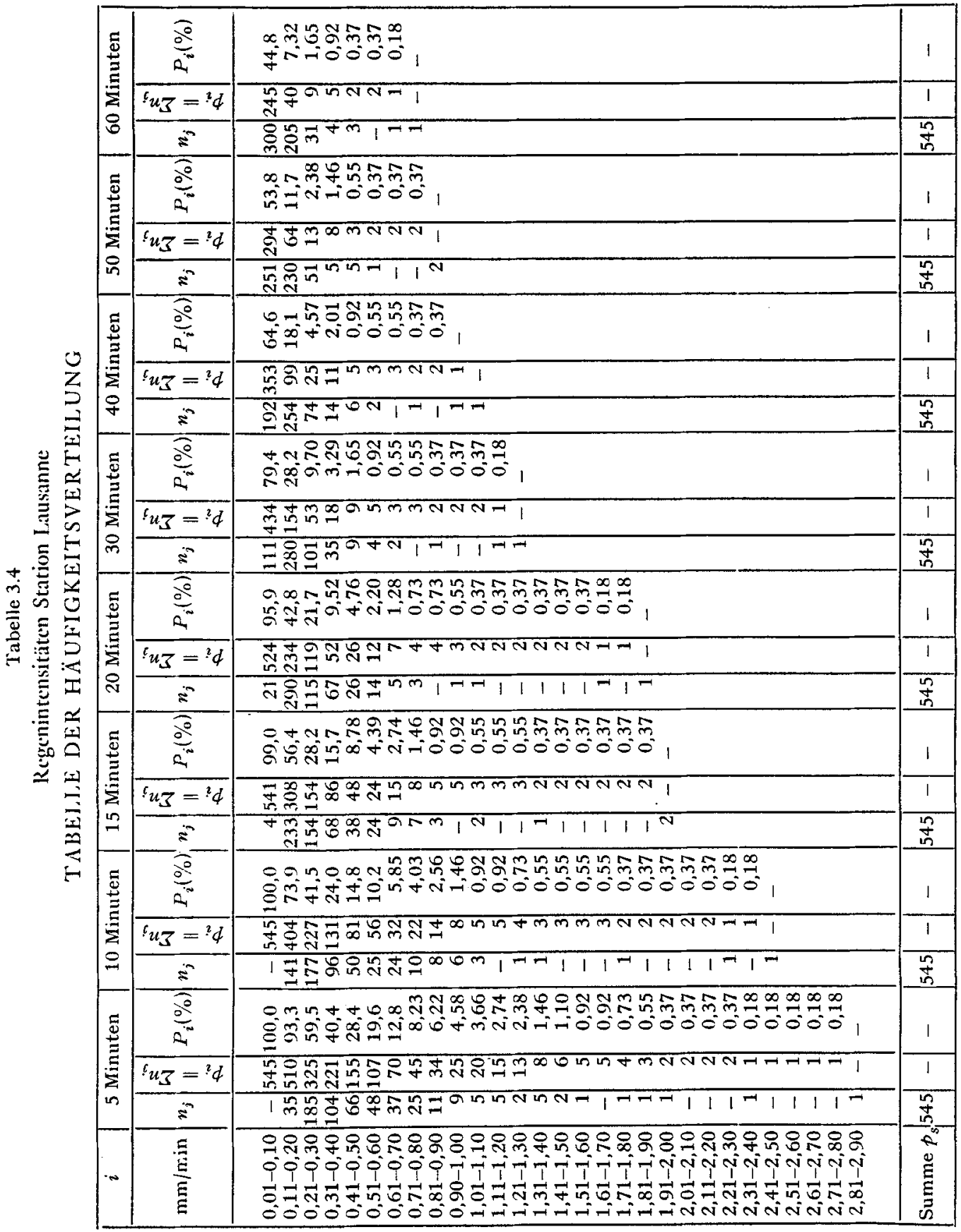




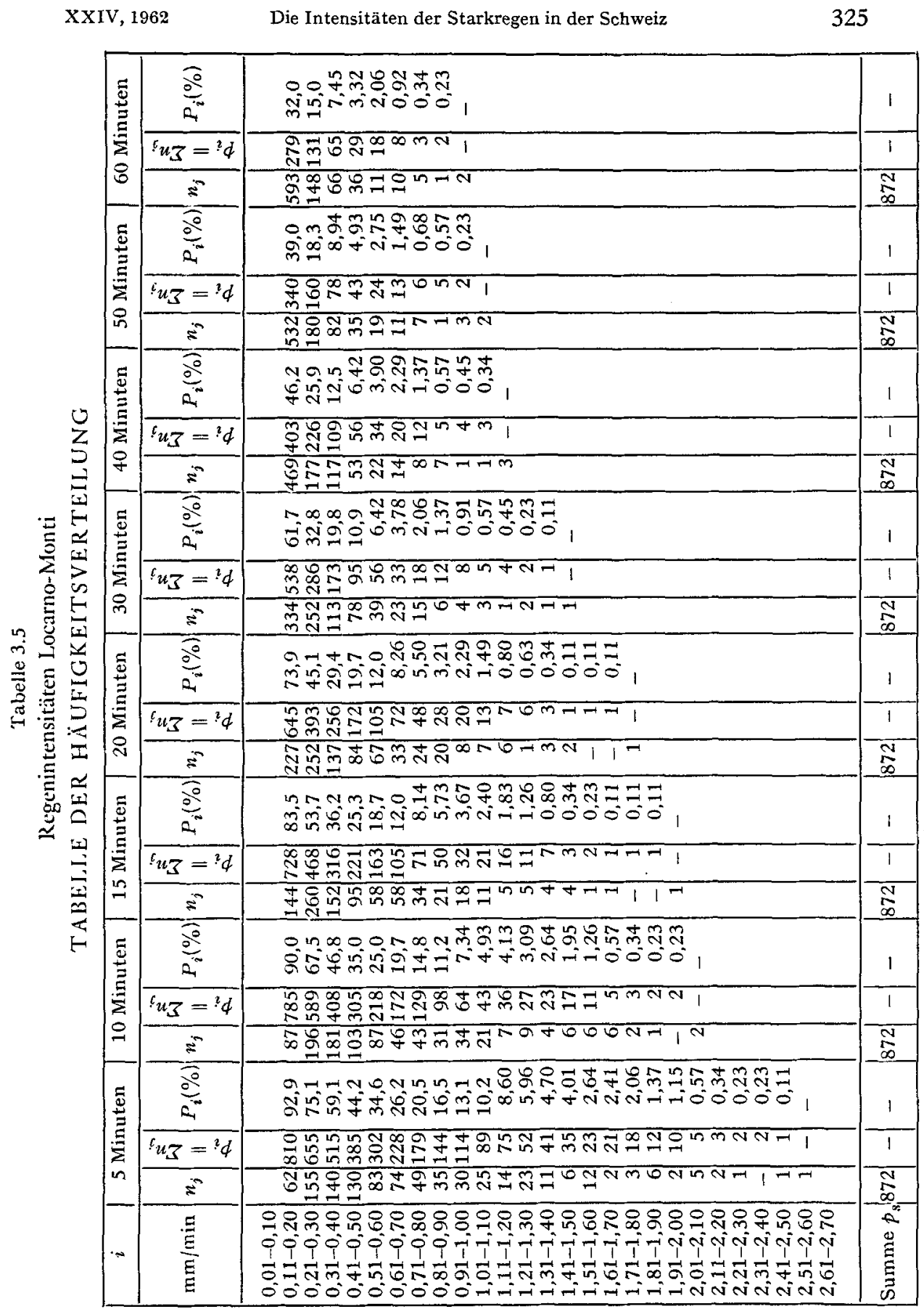




\begin{tabular}{|c|c|c|c|c|}
\hline \multirow{3}{*}{\multicolumn{2}{|c|}{ 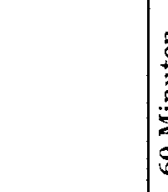 }} & $\frac{a}{9}$ & 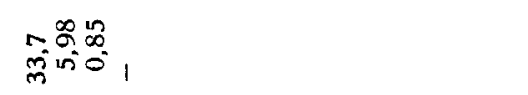 & 1 \\
\hline & & ${ }^{c} u_{3}=?^{2} \phi$ & कूत। & 1 \\
\hline & 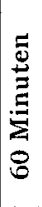 & $=$ & hूुक्नल & 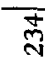 \\
\hline \multirow{13}{*}{ 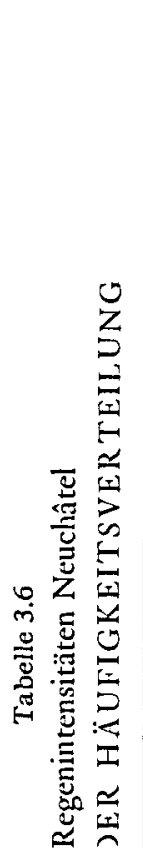 } & \multirow{3}{*}{ 总 } & $\frac{\sqrt{a}}{a^{2}}$ & 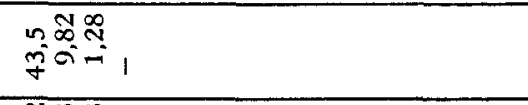 & 1 \\
\hline & & ${ }^{t} u_{3}={ }^{2} \phi$ & बृल & 1 \\
\hline & & $\approx$ & masহ $^{2}$ & m \\
\hline & \multirow{3}{*}{ 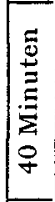 } & $\frac{20}{a+\infty}$ & 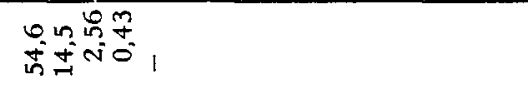 & 1 \\
\hline & & $\overline{{ }^{c} u_{z}={ }^{2} \phi}$ & $\stackrel{\infty}{\Im} \bar{m}^{0-1}$ & 1 \\
\hline & & $\therefore$ & ठ্রু & $\stackrel{\vec{m}}{\mathrm{~m}}$ \\
\hline & \multirow{3}{*}{ 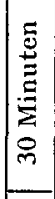 } & $\frac{\widehat{a}}{e^{2}}$ & 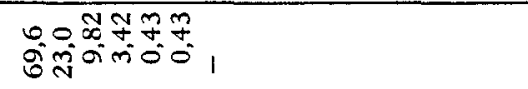 & 1 \\
\hline & & $\overline{{ }^{\prime} u_{Z}={ }^{?} \phi}$ & 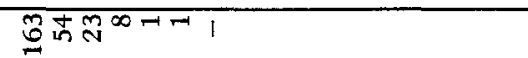 & 1 \\
\hline & & $\approx$ & 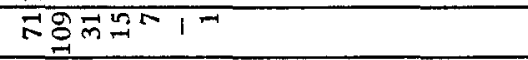 & $\stackrel{f}{3}$ \\
\hline & \multirow{3}{*}{ 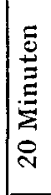 } & $\frac{a}{a}$ & 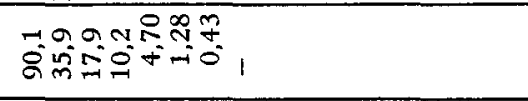 & 1 \\
\hline & & ${ }^{\prime} u_{Z}={ }^{2} \phi$ & 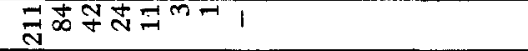 & 1 \\
\hline & & $\therefore$ & งิง ำ & $\stackrel{+}{\theta}$ \\
\hline & \multirow{3}{*}{ } & $\frac{2}{a^{\circ}}$ & 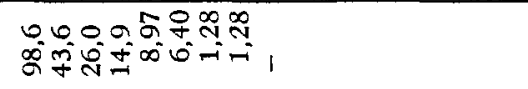 & । \\
\hline$\stackrel{2}{=}$ & & ${ }^{\prime} u_{3}={ }^{i} d$ & 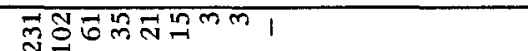 & 1 \\
\hline$\overline{\underline{m}}$ & & $\Leftrightarrow$ & 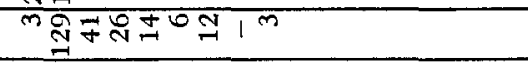 & 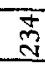 \\
\hline 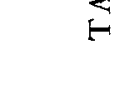 & \multirow{3}{*}{ 总 } & $\frac{\bar{a}}{2 i}$ & 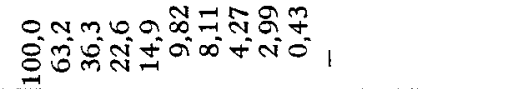 & 1 \\
\hline & & ${ }^{\prime} u_{Z}={ }^{2} \phi$ & 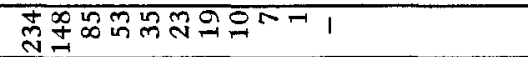 & 1 \\
\hline & & $\therefore$ & 1ষ & $\stackrel{\overbrace{}}{\approx}$ \\
\hline & \multirow{3}{*}{ 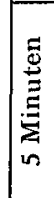 } & $\frac{3}{9}$ & 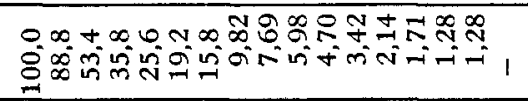 & 1 \\
\hline & & $\varepsilon_{u Z}={ }^{2} \phi$ & 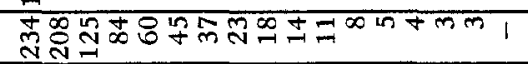 & 1 \\
\hline & & $\approx$ & I & 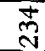 \\
\hline & $\infty$ & $\frac{\stackrel{g}{\xi}}{\underline{\xi}}$ & 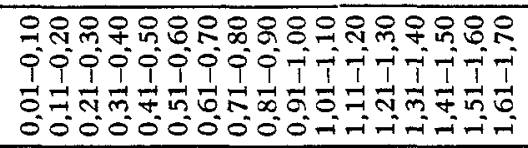 & 芴 \\
\hline
\end{tabular}




\begin{tabular}{|c|c|c|c|c|}
\hline \multirow{3}{*}{\multicolumn{2}{|c|}{ 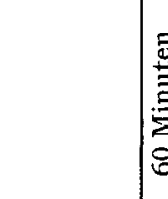 }} & $\frac{a}{0}$ & 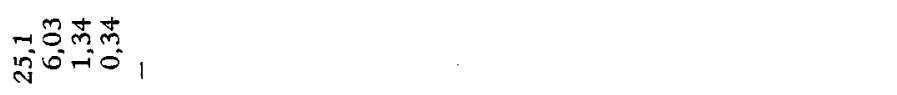 & 1 \\
\hline & & $\overline{e_{u}}={ }^{2} \phi$ & $\underline{n} \infty+\pi \mid$ & 1 \\
\hline & & $\approx$ & $\operatorname{din}_{\text {in }} \pi^{m-1}$ & $\stackrel{\infty}{\$}$ \\
\hline & $\underset{巳}{\stackrel{5}{5}}$ & $\frac{5}{9}$ & 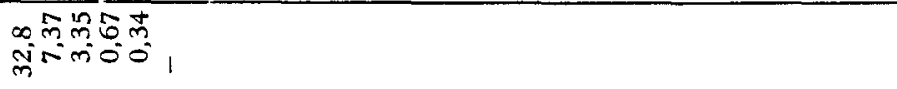 & 1 \\
\hline & 至 & ${ }^{i} u_{\zeta}={ }^{2} \phi$ & $\operatorname{din} 4 \sqrt{4}+1$ & 1 \\
\hline & in & $\approx$ & $\mathbb{N}_{\mathbb{N}} \div \mathbb{N}^{\infty-1}$ & 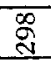 \\
\hline & $\stackrel{5}{ \pm}$ & $\frac{10}{90}$ & 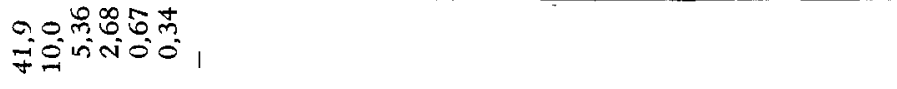 & I \\
\hline$z$ & 妾 & $\epsilon_{u_{3}}={ }^{2} \phi$ & 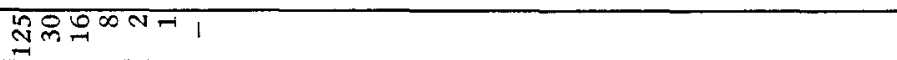 & 1 \\
\hline$\stackrel{D}{=}$ & P & $\approx$ & 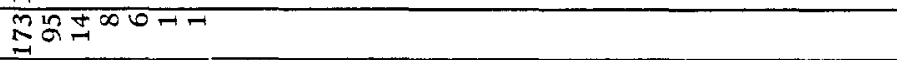 & $\stackrel{\text { M }}{\stackrel{2}{2}}$ \\
\hline$=\frac{G}{\underline{E}}$ & 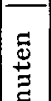 & $\frac{2}{2}$ & 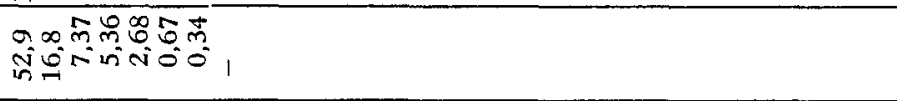 & 1 \\
\hline & $\stackrel{\Xi}{\Sigma}$ & $\iota_{3 Z}={ }^{2} \phi$ & 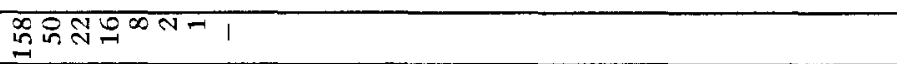 & 1 \\
\hline 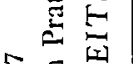 & 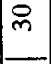 & $\mathrm{s}^{2}$ & $\sum_{0}^{\infty}$ & $\stackrel{\substack{n\\
}}{2}$ \\
\hline $\begin{array}{ll}0 \\
0\end{array}$ & $\stackrel{\Xi}{\Xi}$ & $\frac{5}{a}$ & 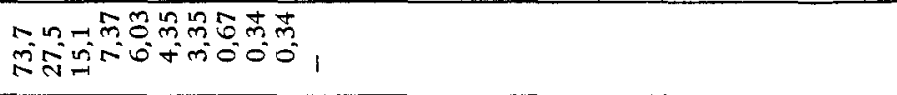 & 1 \\
\hline 㺼: & 灵 & $\overline{c_{u z}={ }^{2} \phi}$ & 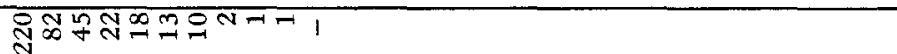 & 1 \\
\hline I & 尺 & $=$ & 冓的啇 & $\stackrel{\infty}{\stackrel{2}{9}}$ \\
\hline 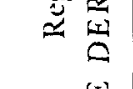 & 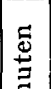 & $\frac{50}{c^{\circ}}$ & 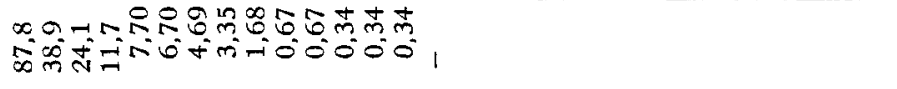 & 1 \\
\hline ב & 离 & ${ }^{{ }^{6} u_{3} z={ }^{2} d}$ & 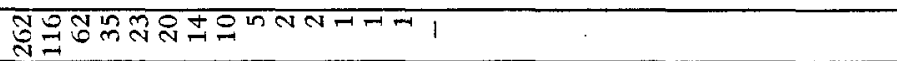 & 1 \\
\hline$\underline{201}$ & $\stackrel{n}{\sim}$ & $\approx$ & 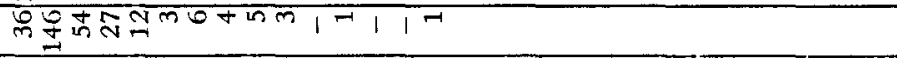 & कू \\
\hline$E$ & $\stackrel{E}{E}$ & $\frac{a}{20}$ & 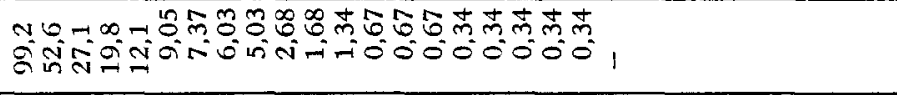 & 1 \\
\hline & $\sum$ & ${ }^{l} u_{3}={ }^{2} \phi$ & 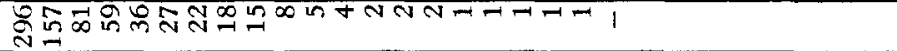 & 1 \\
\hline & $\stackrel{\sim}{-1}$ & $\approx$ & 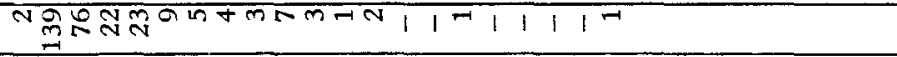 & 今 \\
\hline & 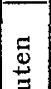 & $\frac{2}{20}$ & 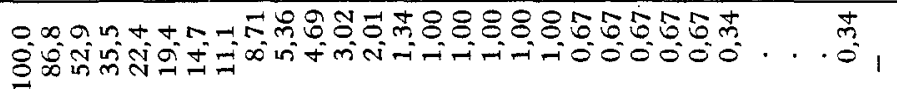 & 1 \\
\hline & $\bar{\Delta}$ & ${ }^{\prime} u_{3}={ }^{2} \phi$ & 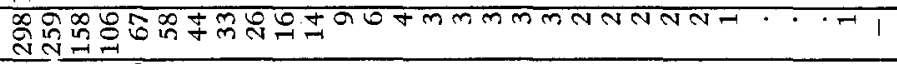 & 1 \\
\hline & in & $\approx$ & 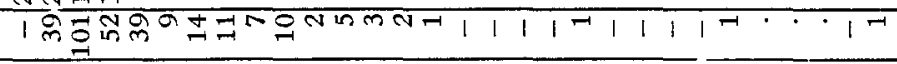 & $\stackrel{\text { }}{\stackrel{2}{2}}$ \\
\hline & $\infty$ & $\frac{\Xi}{g}$ & 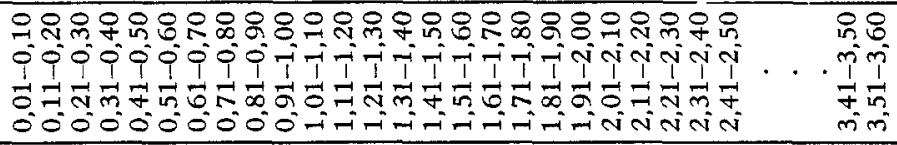 & 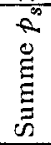 \\
\hline
\end{tabular}




\begin{tabular}{|c|c|c|c|c|}
\hline & \multirow{3}{*}{ 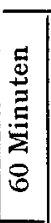 } & $\stackrel{\frac{\partial}{g}}{2}$ & 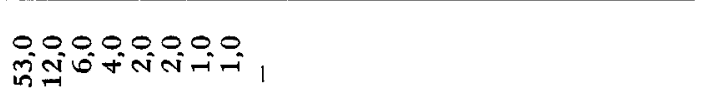 & 1 \\
\hline & & $c_{u z}={ }^{2} d$ & 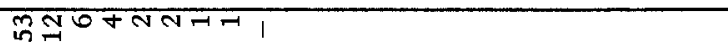 & 1 \\
\hline & & $\approx$ & 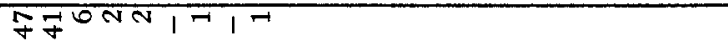 & 8 \\
\hline \multirow{22}{*}{ 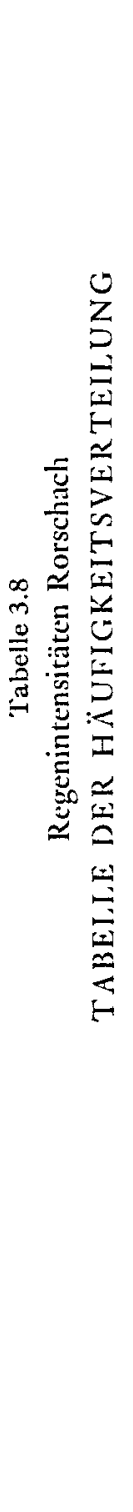 } & \multirow{3}{*}{ 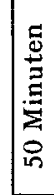 } & $\frac{8}{20}$ & 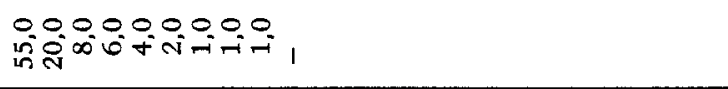 & 1 \\
\hline & & $c_{u z}=I^{2} \phi$ & $\ln 8 \infty 0+4 न 7 न 1$ & 1 \\
\hline & & $\approx$ & $\operatorname{lng} N$ लूलन। & $\stackrel{8}{9}$ \\
\hline & \multirow{3}{*}{ 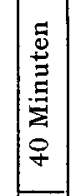 } & $\frac{2}{20}$ & 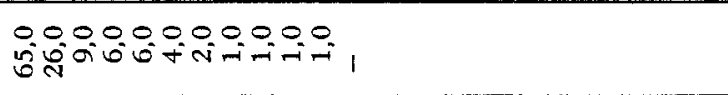 & 1 \\
\hline & & ${ }^{i} u_{3}={ }^{\prime} \phi$ & $\operatorname{lng}_{0} 00+47-7$ & 1 \\
\hline & & $\approx$ & 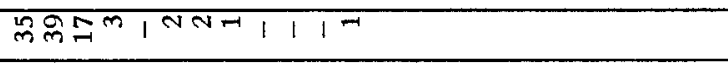 & 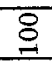 \\
\hline & \multirow{3}{*}{ 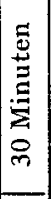 } & $\frac{2}{2}$ & 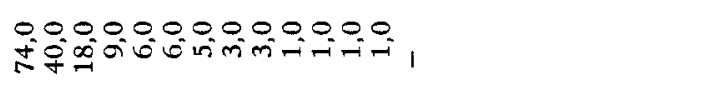 & । \\
\hline & & ${ }^{t} u_{3}={ }^{2} \phi$ & 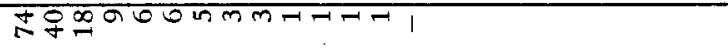 & 1 \\
\hline & & $=$ & 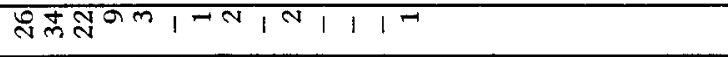 & 8 \\
\hline & \multirow{3}{*}{ 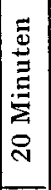 } & $\frac{a}{a}$ & 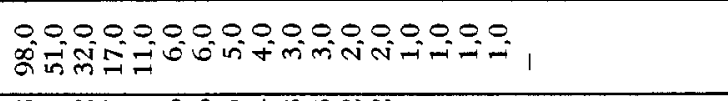 & 1 \\
\hline & & ${ }^{l_{u} u_{3}={ }^{2} \phi}$ & 的 & 1 \\
\hline & & $\approx$ & 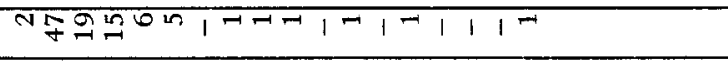 & $\stackrel{8}{8}$ \\
\hline & \multirow{3}{*}{ 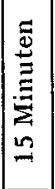 } & $\frac{5}{20}$ & 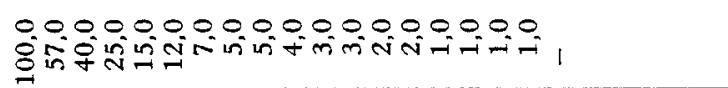 & 1 \\
\hline & & ${ }^{c} u_{K}={ }^{2} \phi$ & 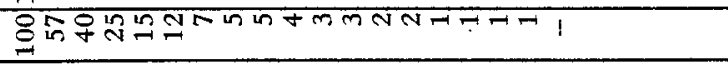 & i \\
\hline & & $\therefore$ & I mangmand & 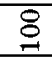 \\
\hline & \multirow{3}{*}{ 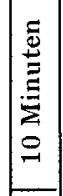 } & $\frac{5}{2}$ & 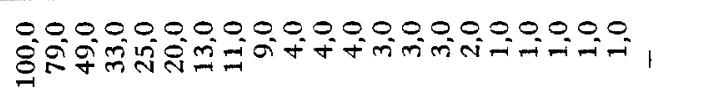 & 1 \\
\hline & & ${ }^{2} z^{u}=c^{c} \phi$ & 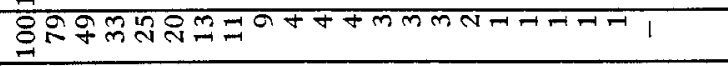 & 1 \\
\hline & & $\approx$ & 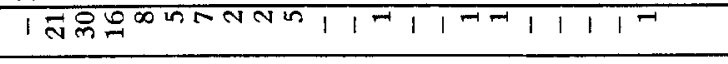 & $\stackrel{3}{8}$ \\
\hline & \multirow{3}{*}{$\mid$} & $\frac{3}{2}$ & 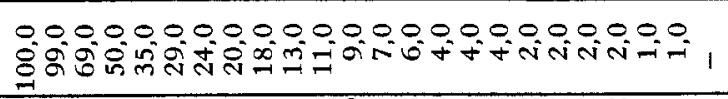 & 1 \\
\hline & & $c_{u_{Z}}={ }^{2} \phi$ & 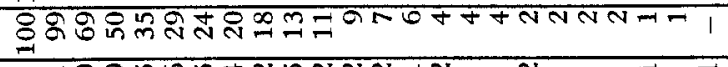 & 1 \\
\hline & & $\approx$ & $I_{\text {mbing }}$ & 8 \\
\hline & $\therefore$ & 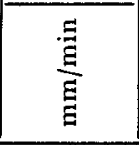 & 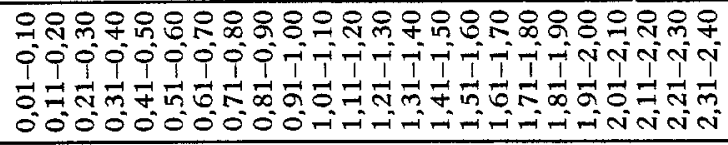 & 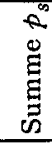 \\
\hline
\end{tabular}


Tabelle 3.9

Regenintensitäten St. Gallen

TABELLE DER HÄUFIGKEITSVERTEILUNG

\begin{tabular}{|c|c|c|c|c|c|c|c|c|c|c|c|c|}
\hline$i$ & \multicolumn{3}{|c|}{10 Minuten } & \multicolumn{3}{|c|}{20 Minuten } & \multicolumn{3}{|c|}{30 Minuten } & \multicolumn{3}{|c|}{60 Minuten } \\
\hline $\mathrm{mm} / \mathrm{min}$ & $n_{j}$ & $\begin{array}{l}-\infty \\
11 \\
\infty\end{array}$ & $P_{i}(\%)$ & $n_{j}$ & $\begin{array}{l}\overrightarrow{\hat{\omega}} \\
\| \\
\tilde{2}\end{array}$ & $P_{i}(\%)$ & $n_{j}$ & 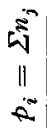 & $P_{i}(\%)$ & $n_{j}$ & $\mid \begin{array}{l}5 \\
11 \\
\therefore\end{array}$ & $P_{i}(\%)$ \\
\hline $0,01-0,10$ & -1 & 599 & 100,0 & 1 & 598 & 9986 & 79 & 520 & 868 & 268 & 331 & 553 \\
\hline $0,11-0,20$ & 74 & 525 & 87,7 & 291 & 307 & 51,3 & 320 & 200 & 33,4 & 252 & 79 & 13,2 \\
\hline $0,21-0,30$ & 234 & 291 & 48,6 & 158 & 149 & 24,9 & 111 & 89 & 14,9 & 54 & 25 & 4,17 \\
\hline $0,31-0,40$ & 124 & 167 & 27,9 & 74 & 75 & 12,5 & 47 & 42 & 7,01 & 9 & 16 & 2,67 \\
\hline $0,41-0,50$ & 61 & 106 & 17,7 & 27 & 48 & 8,02 & 18 & 24 & 4,01 & 9 & 7 & 1,17 \\
\hline $0,51-0,60$ & 28 & 78 & 13,0 & 17 & 31 & 5,18 & 3 & 21 & 3,51 & 4 & 3 & 0,501 \\
\hline $0,61-0,70$ & 27 & 51 & 8,25 & 7 & 24 & 4,01 & 5 & 16 & 2,67 & 2 & 1 & 0,167 \\
\hline $0,71-0,80$ & 10 & 41 & 6,85 & 5 & 19 & 3,17 & 5 & 11 & 1,84 & 1 & - & - \\
\hline $0,81-0,90$ & 12 & 29 & 4,84 & 2 & 17 & 2,84 & 3 & 8 & 1,34 & & & \\
\hline $0,91-1,00$ & 3 & 26 & 4,34 & 6 & 11 & 1,84 & 4 & 4 & 0,668 & & & \\
\hline $1,01-1,10$ & 5 & 21 & 3,51 & 2 & 9 & 1,50 & 3 & 1 & 0,167 & & & \\
\hline $1,11-1,20$ & 4 & 17 & 2,84 & 1 & 8 & 1,34 & 1 & - & - & & & \\
\hline $1,21-1,30$ & 5 & 12 & 2,00 & 4 & 4 & $.0,668$ & & & & & & \\
\hline $1,31-1,40$ & 3 & 9 & 1,50 & 2 & 2 & 0,334 & & & & & & \\
\hline $1,41-1,50$ & 1 & 8 & 1,34 & 1 & 1 & 0,167 & & & & & & \\
\hline $1,51-1,60$ & 3 & 5 & 0,835 & 1 & - & - & & & & & & \\
\hline $1,61-1,70$ & 1 & 4 & 0,668 & & & & & & & & & \\
\hline $1,71-1,80$ & - & 4 & 0,668 & & & & & & & & & \\
\hline $1,81-1,90$ & - & 4 & 0,668 & & & & & & & & & \\
\hline $1,91-2,00$ & - & 4 & 0,668 & & & & & & & & & \\
\hline $2,01-2,10$ & - & 4 & 0,668 & & & & & & & & & \\
\hline $2,11-2,20$ & - & 4 & 0,668 & & & & & & & & & \\
\hline $2,21-2,30$ & 3 & 1 & 0,167 & & & & & & & & & \\
\hline $2,31-2,40$ & 1 & - & - & & & & & & & & & \\
\hline $2,41-2,50$ & - & - & - & & & & & & & & & \\
\hline $2,51-2,60$ & - & - & - & & & & & & & & & \\
\hline $2,61-2,70$ & - & - & - & & & & & & & & & \\
\hline $2,71-2,80$ & - & - & - & & & & & & & & & \\
\hline Summe $p_{s}$ & 99 & - & - & 599 & - & - & 599 & - & - & 599 & - & - \\
\hline
\end{tabular}




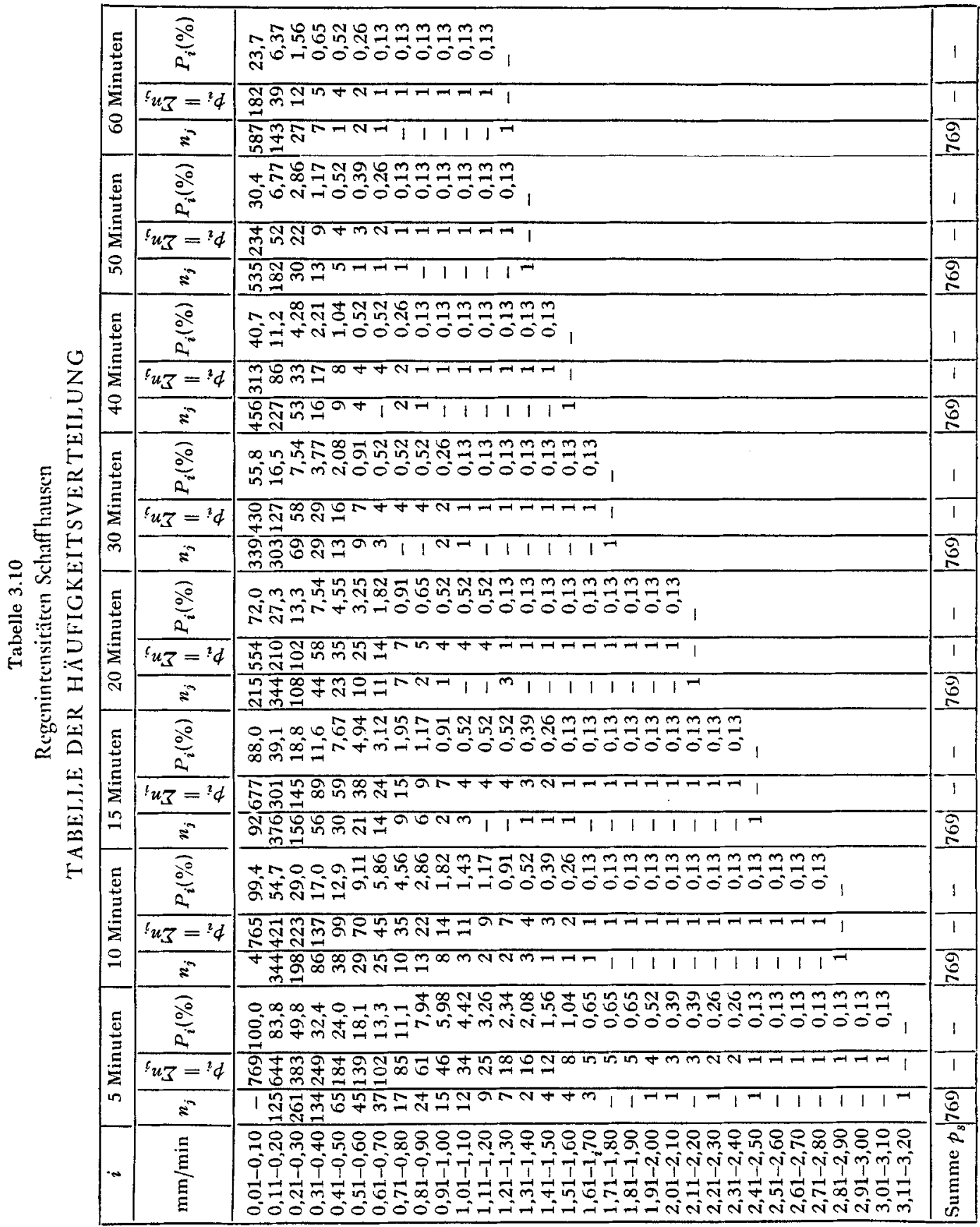




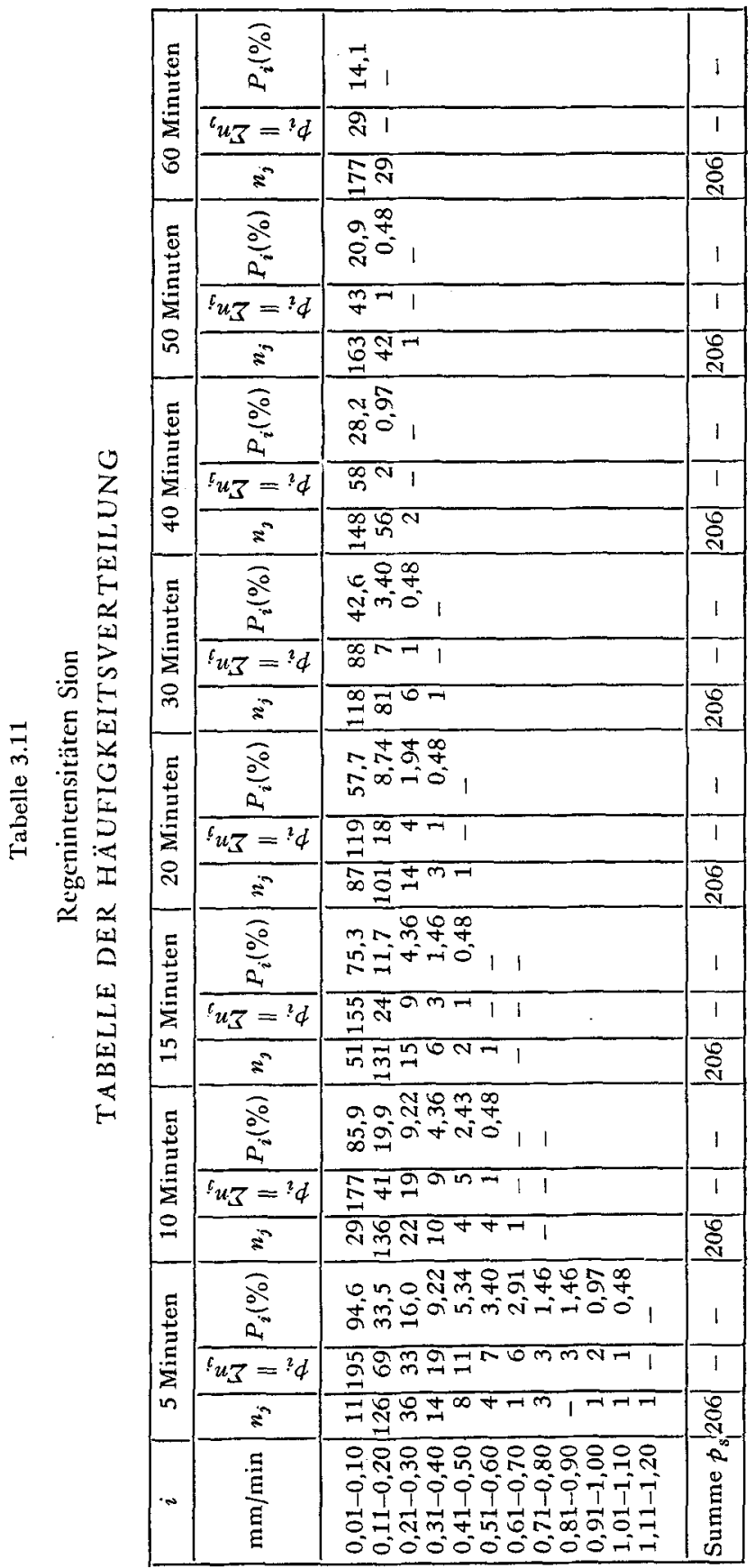




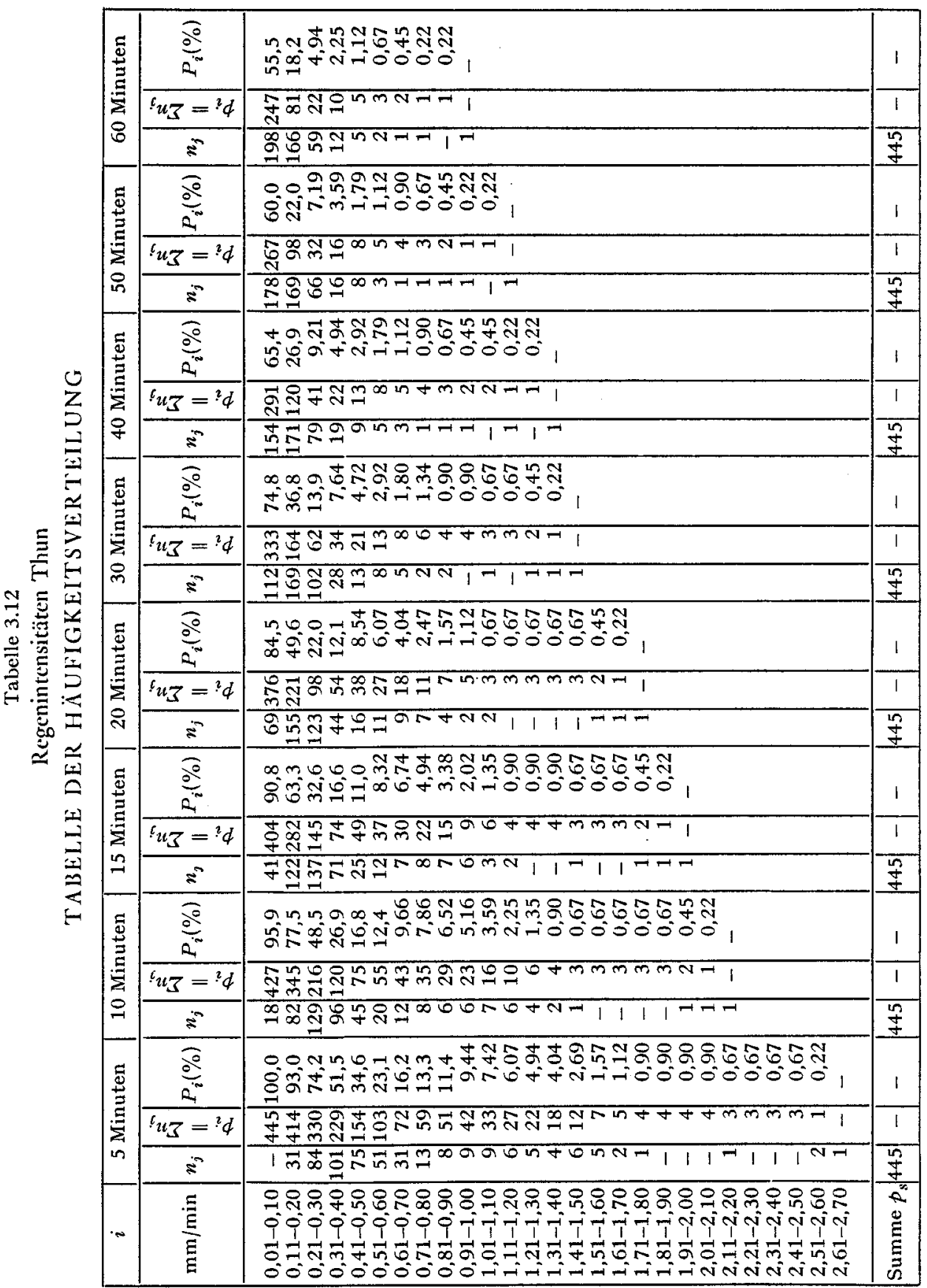




\begin{tabular}{|c|c|c|c|c|}
\hline \multirow{3}{*}{\multicolumn{2}{|c|}{ 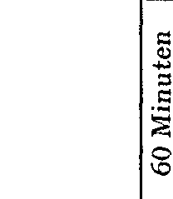 }} & $\frac{5}{8}$ & 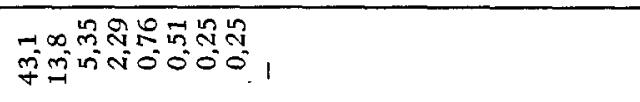 & । \\
\hline & & $\overline{l_{u z}=? d}$ & 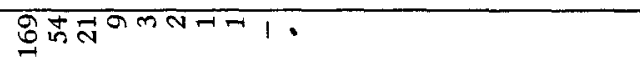 & 1 \\
\hline & & $\approx$ & 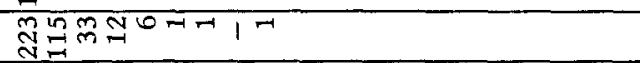 & di \\
\hline \multirow{9}{*}{\multicolumn{2}{|c|}{ 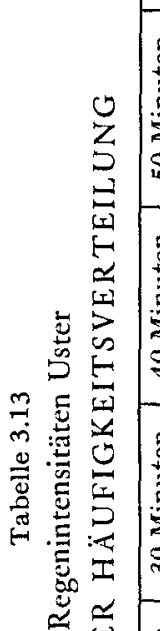 }} & $\frac{2}{20}$ & 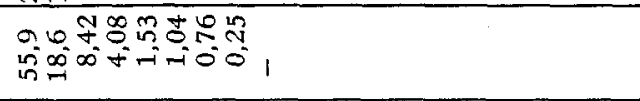 & 1 \\
\hline & & $l_{u z}=?^{\prime} d$ & $\sum_{n}^{2 m} m 0+m-1$ & 1 \\
\hline & & $\approx$ & 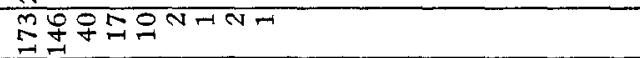 & gू \\
\hline & & $\frac{10}{2}$ & 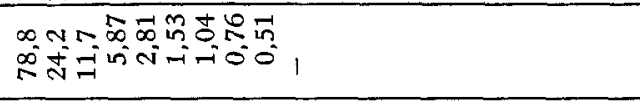 & 1 \\
\hline & & $\overline{t_{u z}={ }^{2} \phi}$ & 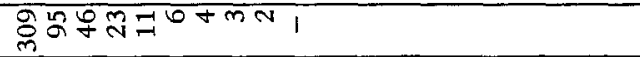 & 1 \\
\hline & & $\approx$ & 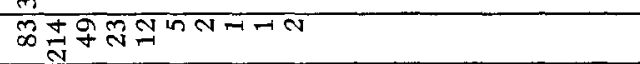 & S్ \\
\hline & & $\frac{2}{2}$ & 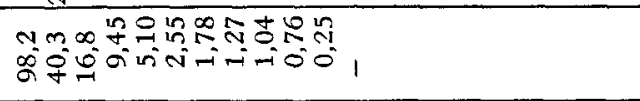 & 1 \\
\hline & & ${ }_{u z}={ }^{i} \phi$ & 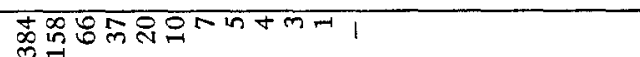 & 1 \\
\hline & & $\approx$ & 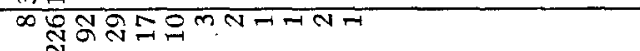 & लू \\
\hline$\simeq \underset{\frac{1}{0}}{\simeq}$ & \multirow{3}{*}{ 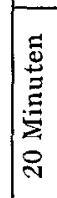 } & $\frac{30}{20}$ & 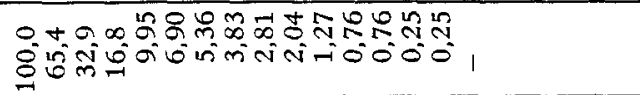 & 1 \\
\hline$\stackrel{15}{=}$ & & ${ }^{c} u_{K}={ }^{?} \phi$ & ปู่ & 1 \\
\hline$\underline{\underline{n}}$ & & $\approx$ & 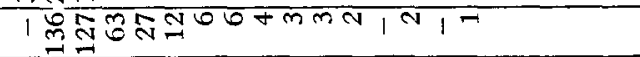 & ๙ু \\
\hline$\underline{E}$ & 离 & $\frac{20}{6 i}$ & 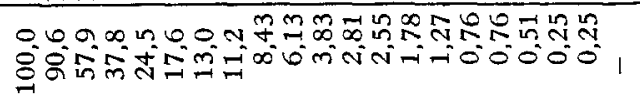 & 1 \\
\hline & 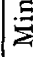 & $\epsilon_{u_{K}}={ }^{2} \phi$ & 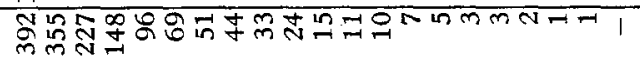 & 1 \\
\hline & $\stackrel{1}{2}$ & $\therefore$ & | & אू \\
\hline & $\rightarrow$ & $\frac{\stackrel{\Xi}{\Xi}}{\stackrel{\Xi}{\Xi}}$ & 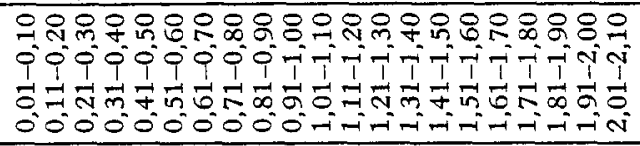 & है \\
\hline
\end{tabular}




\begin{tabular}{|c|c|c|c|c|}
\hline & \multirow{3}{*}{ 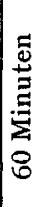 } & $\frac{9}{20}$ & 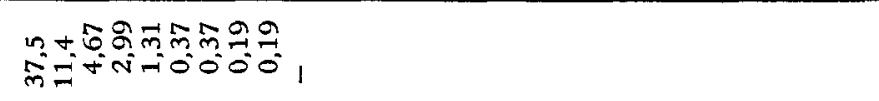 & I \\
\hline & & $\overline{c_{u z}={ }^{2} \phi}$ & 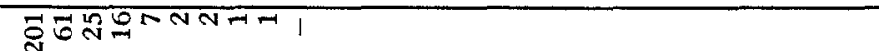 & 1 \\
\hline & & $\approx$ & 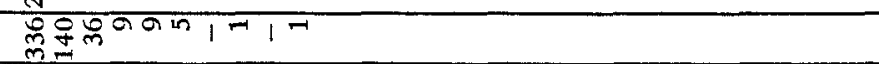 & in \\
\hline & $\stackrel{\Xi}{\Xi}$ & $\frac{3}{20}$ & 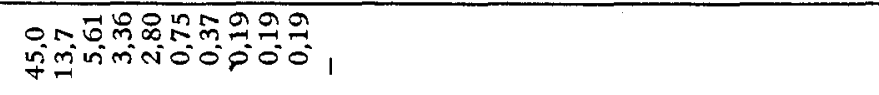 & । \\
\hline & 寻 & ${ }^{c_{u}}={ }^{2} \phi$ & 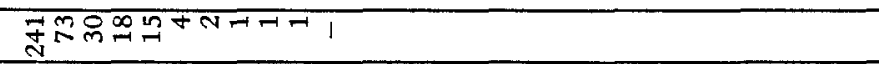 & 1 \\
\hline & & $\approx$ & 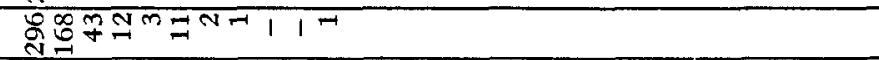 & 空 \\
\hline & $\stackrel{E}{\stackrel{E}{\Xi}}$ & $\frac{5}{20}$ & 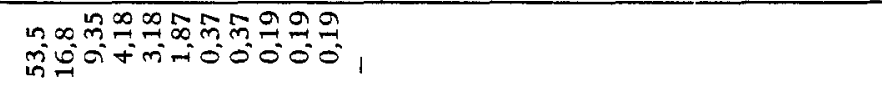 & । \\
\hline$z$ & E & $\overline{c_{u z}={ }^{2} \phi}$ & 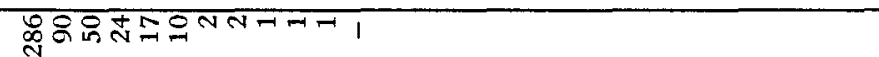 & 1 \\
\hline$\stackrel{Q}{\Xi}$ & P & $\approx$ & 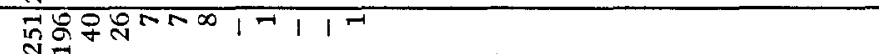 & 裔 \\
\hline$\stackrel{\Xi}{\cong}$ & 站 & $\frac{\partial}{a}$ & 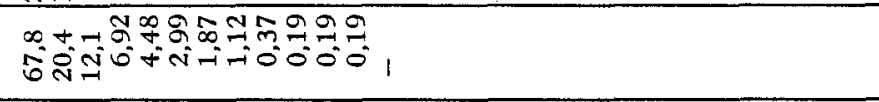 & 1 \\
\hline 矛施 & 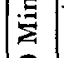 & ${ }^{c_{u z}}={ }^{2} \phi$ & modog & 1 \\
\hline 空 & קి & $\approx$ & 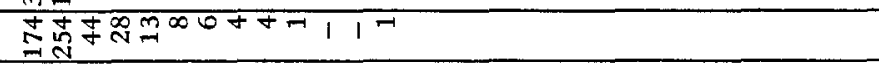 & in \\
\hline 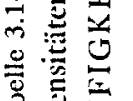 & $\frac{\Xi}{\vec{E}}$ & $\frac{\bar{a}}{a^{2}}$ & 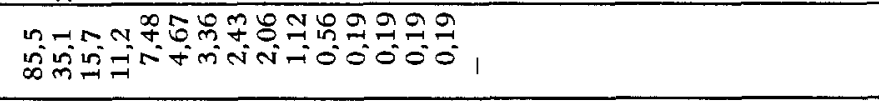 & 1 \\
\hline 茪: & 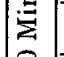 & ${ }^{\imath} u_{3}={ }^{2} \phi$ & 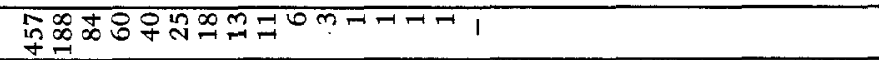 & 1 \\
\hline I & 尺ి & $\approx$ & 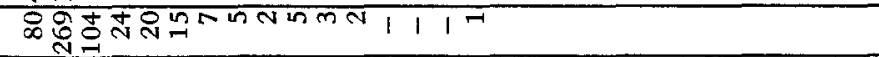 & $\overline{\tilde{n}}$ \\
\hline 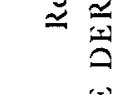 & 咅 & $\frac{3}{9}$ & 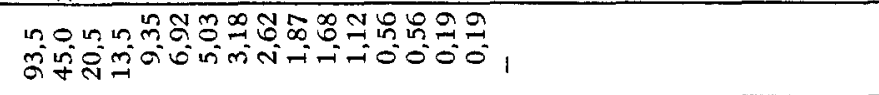 & । \\
\hline$\Xi$ & 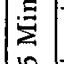 & $e_{u z}={ }^{2} d$ & 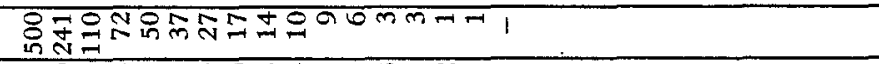 & 1 \\
\hline 空 & $\stackrel{2}{\rightarrow}$ & in & m & 颌 \\
\hline$\stackrel{4}{H}$ & 矛 & $\frac{20}{20}$ & 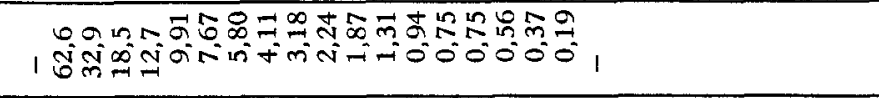 & । \\
\hline & $E$ & $\overline{c_{u z}={ }^{2} d}$ & 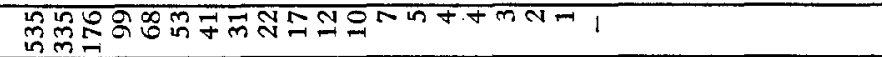 & 1 \\
\hline & $\stackrel{9}{-1}$ & $\approx$ & 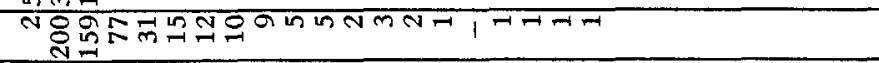 & in \\
\hline & $\stackrel{\Xi}{\stackrel{5}{\Xi}}$ & $\frac{30}{2}$ & 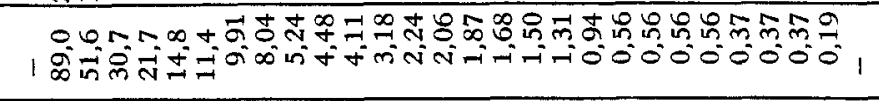 & 1 \\
\hline & $\Xi$ & ${ }^{e} u_{3}={ }^{2} \phi$ & 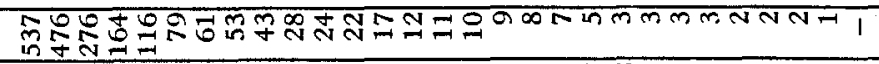 & 1 \\
\hline & in & $\approx$ & 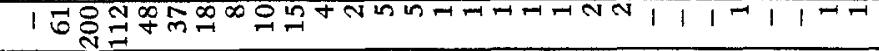 & $\stackrel{n}{n}$ \\
\hline & $\infty$ & $\frac{\Xi}{\xi}$ & 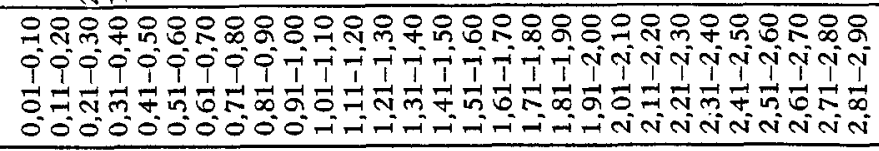 & $\stackrel{2}{E}$ \\
\hline
\end{tabular}




\begin{tabular}{|c|c|c|c|c|}
\hline \multirow{3}{*}{\multicolumn{2}{|c|}{ 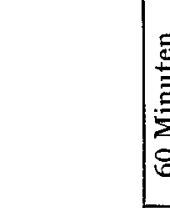 }} & $\frac{8}{8}$ & 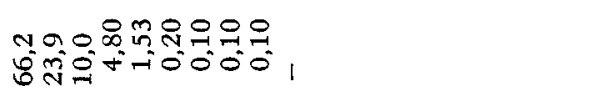 & 1 \\
\hline & & ${ }^{{ }^{c} u_{z}={ }^{?} \phi}$ & 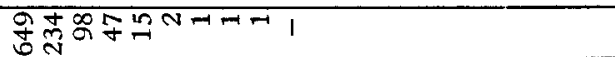 & 1 \\
\hline & & $\approx$ & 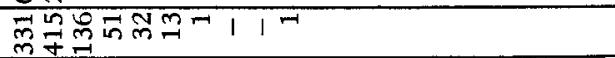 & $\stackrel{8}{\circ}$ \\
\hline & 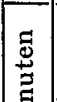 & $\frac{\sqrt[3]{6}}{i^{i}}$ & 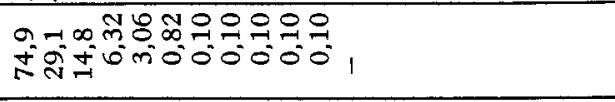 & 1 \\
\hline & 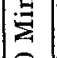 & ${ }^{c} u_{Z}={ }^{2} \phi$ & 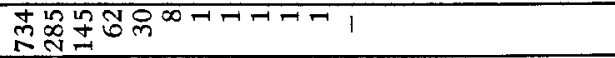 & 1 \\
\hline & & $\approx$ & 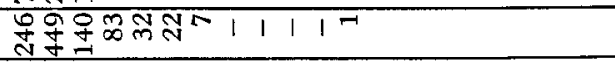 & $\stackrel{8}{\circ}$ \\
\hline & $\stackrel{\Xi}{ \pm}$ & $\frac{5}{\sigma^{\circ}}$ & 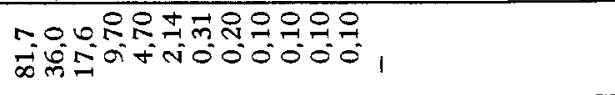 & । \\
\hline & 5 & $\overline{{ }^{6} u_{z}={ }^{?} \phi}$ & 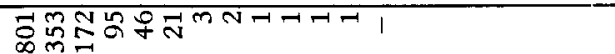 & 1 \\
\hline & fo & $\approx$ & 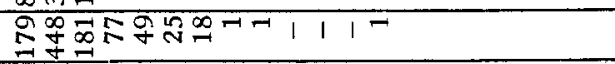 & $\stackrel{8}{\circ}$ \\
\hline 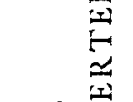 & $\stackrel{\Xi}{\Xi}$ & $\frac{\partial}{a^{2}}$ & 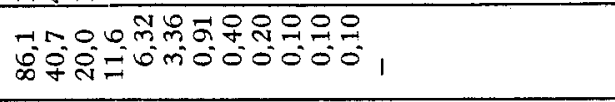 & । \\
\hline$\stackrel{一}{\Xi}$ & 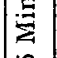 & ${ }^{c_{u z}}={ }^{2} \phi$ & + & 1 \\
\hline $8 \stackrel{0}{5}$ & $\ln$ & $\approx$ & 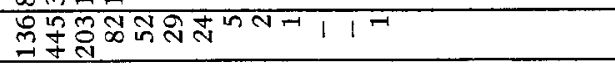 & $\stackrel{2}{2}$ \\
\hline 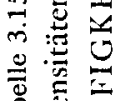 & 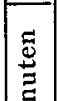 & $\frac{2}{20}$ & 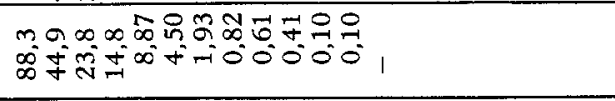 & । \\
\hline 总: & 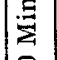 & ${ }^{\prime} u_{\zeta}={ }^{2} \phi$ & 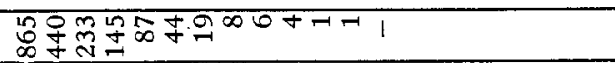 & 1 \\
\hline$\underbrace{5}_{3}=$ & 凩 & $\approx$ & 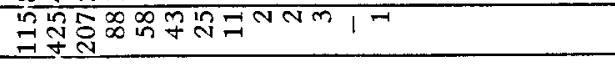 & $\stackrel{8}{\circ}$ \\
\hline$\approx \stackrel{\simeq}{\simeq}$ & 焉 & $\frac{\pi}{20}$ & 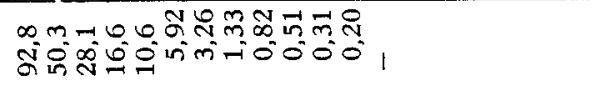 & । \\
\hline$\stackrel{2}{\Xi}$ & 榁 & $\iota_{u z}=?^{\prime} \phi$ & 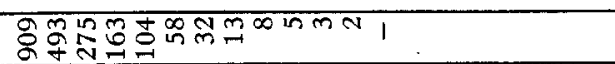 & 1 \\
\hline 产 & 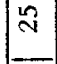 & $\approx$ & 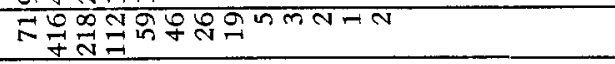 & $\stackrel{\circ}{\circ}$ \\
\hline$\sum$ & 竭 & $\frac{2}{2}$ & 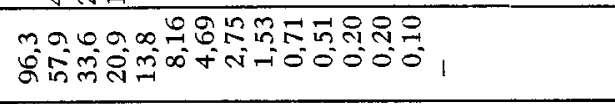 & । \\
\hline & $E$ & ${ }^{l_{u z}}={ }^{?} d$ & 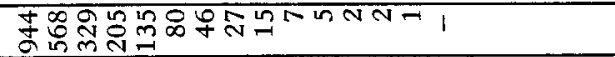 & 1 \\
\hline & ஜิ & $\therefore$ & 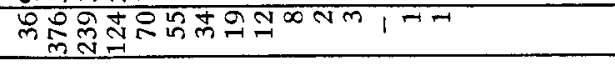 & $\begin{array}{l}0 \\
\infty \\
\infty\end{array}$ \\
\hline & 咅 & $\frac{2}{5}$ & 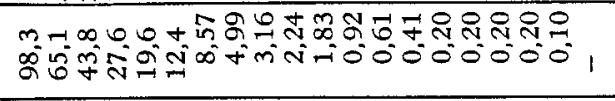 & 1 \\
\hline & E & ${ }^{{ }^{\prime} u_{3}={ }^{2} d}$ & 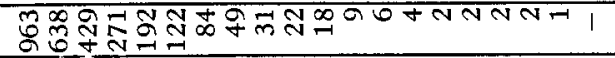 & 1 \\
\hline & $=$ & $=$ & 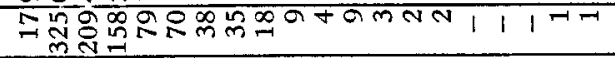 & 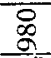 \\
\hline & $\cdot$ & 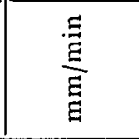 & 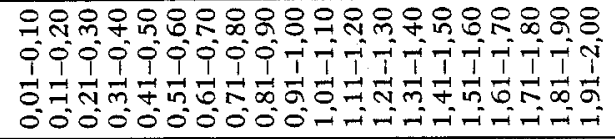 & 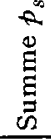 \\
\hline
\end{tabular}


mit der Regendauer formelmässig darstellen, wäre die Formel für die Regenintensität derart kompliziert, dass sie für die Praxis unbrauchbar würde (Regendauer $T$ im Exponenten einer Exponentialfunktion). Ein Versuch, eine Annäherung zu finden, indem der Wert $B$ mit der Häufigkeit variiert wird, führte zu keinem verwendbaren Resultat. Es wird unumgänglich sein, nach Vorliegen einer genügend langen Beobachtungszeit, die Auswertung für Sion zu ergänzen.

\subsection{Beziebung zwiscben den Zentralwerten und der Regendauer}

Bei allen ausgewerteten Regenstreifen ergab sich eine lineare Beziehung zwischen den reziproken Zentralwerten, wie sie aus den Summenhäufigkeitsdiagrammen herausgelesen wurden und der Regendauer $T$ in Minuten und zwar wurde $\xi=A / T+B$, wobei die Werte $A$ als reziproker Tangens ( $=$ Cotangens) des Steigungswinkels der Geraden und $B$ als Abschnitt auf der Abszissenachse aus den Diagrammen herausgelesen werden können. Auf eine Darstellung der Diagramme selbst sei verzichtet. Hingegen sind in der Tab. 4 die erhaltenen Werte $A$ und $B$ wiedergegeben.

\subsection{Die Häufigkeitsfaktoren b}

Die Häufigkeitsfaktoren, die nur von den Jahren $\approx$ abhängen, ergeben sich indirekt aus dem Summenhäufigkeitsdiagramm. Sie sind somit abhängig vom Verlauf der schiefen Häufigkeitsverteilung, bzw. von der Umkehrfunktion des Gaußschen Integrals, das als Exponent einer Exponentialfunktion mit der Basis $e=2,71828$ auftritt. Die Gleichung des Häufigkeitsfaktors lautet

$$
b=\mu e^{\sigma \Psi(\lambda / z)}, \quad \text { wobei } \lambda=\frac{S}{p_{s}}, \quad \mu=e^{-\sigma \Psi(\lambda)} .
$$

Nach Definition (siehe auch Abschnitt 6.4) soll der Häufigkeitsfaktor tür die einjährige Häufigkeit $(n=z=I)$ zu eins werden. Daraus bestimmt sich der oben gegebene Ausdruck für den Koeffizienten $\mu$.

Für die einzelnen Meßstationen ergaben sich die in der Tab. 4 aufgeführten Werte für die Konstanten.

Über diese Häufigkeitsfaktoren sind in der Literatur mehrfache Hinweise vorhanden. Für die Regenintensitäten der Schweiz wurde unseres Wissens bisher nur ein einziger Versuch einer formelmässigen Beziehung der Häufigkeitsfaktoren bekanntgegeben. Gewisse Regenintensitäts- 
Tabelle 4

Tabelle der Konstanten:

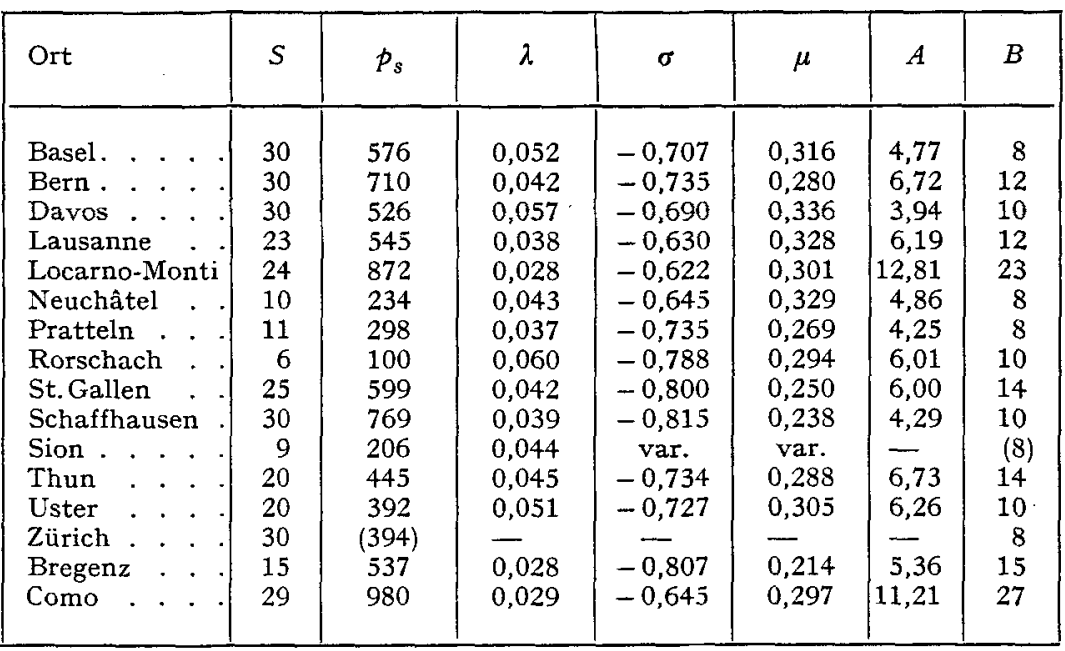

kurven, die heute verwendet werden, würden übrigens keine einwandfreie mathematische Beziehung ergeben. Auf Grund der Wahrscheinlichkeitsrechnung besteht jedoch zweifellos ein solches Verteilungsgesetz für die Häufigkeit.

Bereits GoODRICH [I5] gelangt auf Grund ähnlicher Betrachtungen wie vorstehend aufgeführt zu analogen Ergebnissen. REINHOLD [s] hat für Deutschland die Beziehung $b=z^{0,35}$ aufgestellt, die er jedoch nur für den Bereich $0,5 \leq \approx \leq$ Io als gültig erachtet. In erster Annäherung kann somit die Regenintensitätskurve, die alle $\approx$ Jahre einmal erreicht oder überschritten wird, aus der einjährigen Kurve durch Multiplikation mit der dritten Wurzel aus z gefunden werden. Eine weitere Beziehung REINHOLDS ergibt sich für den Häufigkeitsfaktor $z u b=\mathrm{I}, 58(\sqrt[4]{z}-0,369)$, die jedoch auf den Bereich $\mathrm{I} / 3 \leq z \leq 5$ beschränkt wird. HörLER gab für Zürich die angenäherte Beziehung $b=\sqrt[4]{z}$ bekannt. Für die Häufigkeit von Hochwasserabflüssen wird in den Vereinigten Staaten die interessante Formel von FULLER (zit. in [8]) verwendet, mit dem Häufigkeitsfaktor $b=\mathrm{I}+0,8 \cdot \log z$.

Diese verschiedenen, obenerwähnten Häufigkeitsfaktoren sind in der Abbildung 6 zusammengestellt. Zum Vergleich ist in dieser Abbildung auch der auf Grund der Wahrscheinlichkeitstheorie ermittelte Häufigkeitsfaktor der Stadt Bern aufgeführt. Aus allen diesen Betrachtungen 
Häufigkeitsfaktor $h$

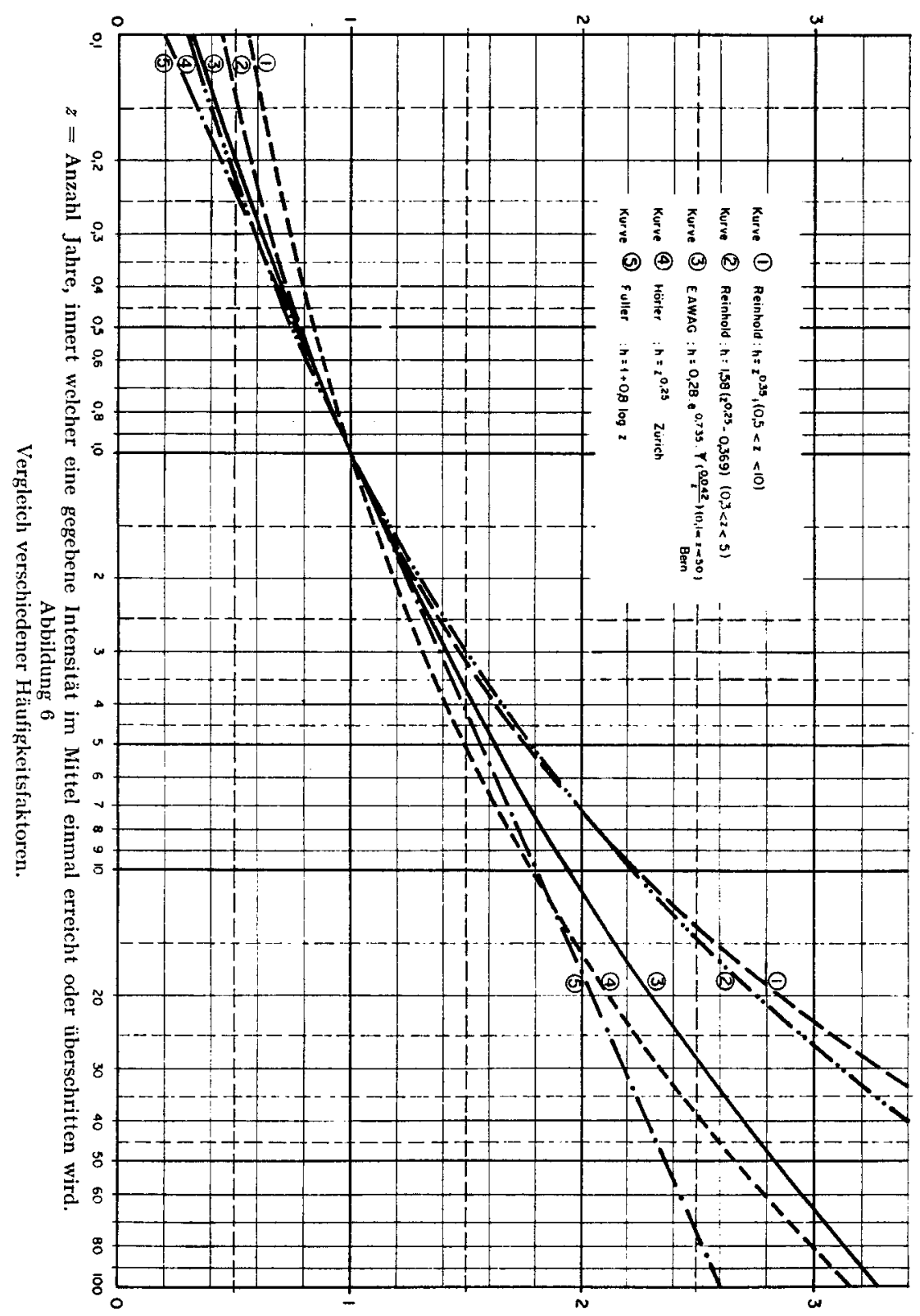


geht hervor, dass sowohl Beobachtungsergebnisse als auch theoretische Berechnungen darauf hindeuten, dass der Häufigkeitsfaktor eine Folge der Gesetze der mathematischen Statistik ist. Wie der theoretisch ermittelte Häufigkeitsfaktor mit den tatsächlichen Messwerten übereinstimmt, geht aus Abbildung 7 hervor, die für Bern durch Abzählen gewonnenen Werte und die theoretischen Kurven zeigt.

\section{S Genauigkeit der Auswertung}

In diesem Abschnitt wird an die Ausführungen in 4.6 angeknüpft. Es dürfte von Interesse sein, wie gross die möglichen Abweichungen der vorgelegten Formeln von der Wirklichkeit sein können. Allerdings benötigt die Ermittlung der relativen Fehler einen verhältnismässig grossen Aufwand an Rechenarbeit. Die Fehlerberechnung wurde deshalb nur für vier typische Stationen durchgeführt, die in gewissem Sinn Grenzfälle darstellen. Es wurden herangezogen:

a) Bern, als Musterbeispiel und typische Mittellandstation mit langer Beobachtungsperiode.

b) Neuchâtel, als Station mit sehr kurzer Beobachtungsperiode.

c) Davos, als Station mit relativ geringen Intensitäten.

d) Locarno-Monti, als Station mit grossen Intensitäten.

Für die übrigen Stationen dürften die Fehler von der gleichen Grösse sein, wie sie durch die obengenannten abgesteckt werden. Ebenso wurden die Berechnungen nicht für alle acht Regendauerstufen (mit Ausnahme von Bern als Beispiel in 4.6), sondern nur für $T=5$, I5, 30 und $60 \mathrm{Mi}$ nuten als nötig erachtet.

Die erhaltenen Resultate sind in der nachfolgenden Zusammenstellung enthaiten.

Relativer Fehler in \%:

\begin{tabular}{|l|c|c|c|c|c|}
\hline \multirow{2}{*}{ Ort } & \multicolumn{3}{|c|}{ Für eine Regendauer von } & $\begin{array}{c}\text { Ungefährer } \\
\text { Gesamtfehler } \\
\text { (relativ in \%) }\end{array}$ \\
\cline { 2 - 5 } & $\mathbf{5 m i n}$ & $15 \mathrm{~min}$ & $30 \mathrm{~min}$ & $60 \mathrm{~min}$ & \\
\hline & & & & & \\
Bern & 6,23 & $\mathbf{5 , 3 0}$ & 9,41 & 9,14 & 6,47 \\
Neuchâtel & 9,12 & 9,58 & 8,95 & 7,28 & 9,17 \\
Davos & 4,15 & $\mathbf{5 , 3 6}$ & 8,67 & 2,64 & 5,62 \\
Locarno-Monti & $\mathbf{8 , 0 7}$ & $\mathbf{8 , 3 7}$ & 6,61 & 4,90 & 7,56 \\
\hline
\end{tabular}




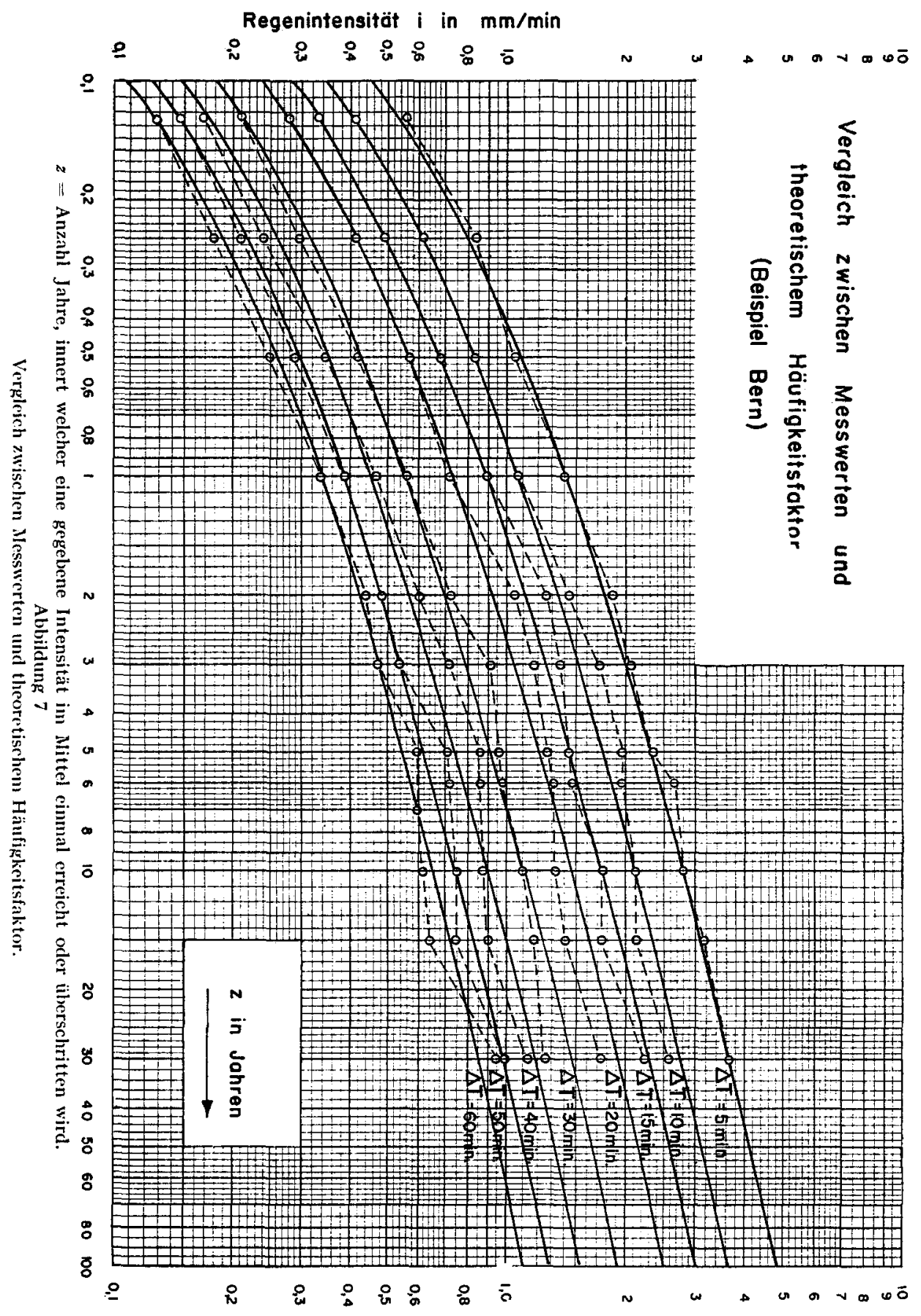


Als Ergebnis kann festgehalten werden, dass bei kurzen Beobachtungsperioden (Neuchâtel) die Auswertung merkbar weniger genau wird. Es scheint auch, dass für Stationen mit grösseren Intensitäten ebenfalls die Fehler eher etwas grösser sind. Im gesamten sind allerdings die Unterschiede zwischen den Fehlern der einzelnen Stationen nicht bedeutend.

\section{Zusammenfassung der Ergebnisse}

\section{I Gleichung der Regenintensitätskurven für die praktischen B̊dürfnisse}

Die Gleichung für die Schar der Regenintensitätskurven kann, wie sich auch theoretisch ergibt, für praktische Zwecke in drei Faktoren zerlegt werden, nämlich in

$$
r=G \varphi b \text {, }
$$

wobei $\quad r=r_{T}^{(z)}=$ Regen von $T$ Minuten Dauer, dessen Intensität im Durchschnitt alle $\approx$ Jahre einmal erreicht oder überschritten wird.

$G=$ Grundzahl $=r_{15}^{(1)}=$ Regen von Is Minuten Dauer, dessen Intensität durchschnittlich alle Jahre einmal zu erwarten ist.

$\varphi=\varphi_{T}=$ Zeitbeiwert nach Imhoff [16], ergibt die Abhängigkeit der Intensität von der Regendauer $T . \varphi$ ist nur von $T$ abhängig.

$b: \therefore b_{z}=$ Häufigkeitsfaktor, ergibt den Abhängigkeitsfaktor der Intensität von der Anzahl Jahre $z$, innert welchen für eine bestimmte Regendauer $T$ die Intensität einmal erreicht oder überschritten wird. $b$ ist nur von $\approx$ abhängig.

In den nachfolgenden Abschnitten werden die einzelnen Faktoren für die verschiedenen Meßstellen übersichtlich zusammengestellt.

\subsection{Die Grundzablen $G$}

Dic in der Literatur als Grundzahlen bezeichneten Werte $G$, also diejenigen Regenintensitäten, die Is Minuten lang andauern und jährlich einmal zu erwarten sind, können aus der nachfolgenden Tabelle für die ausgewerteten Beobachtungsstationen herausgelesen werden. 
Tabelle 5

\begin{tabular}{|c|c|c|c|}
\hline Ort & $\begin{array}{c}G \\
1 / \mathrm{s} \mathrm{ha}\end{array}$ & $\begin{array}{c}B \\
\text { Minuten }\end{array}$ & c \\
\hline.. & 125 & 8 & 0,93 \\
\hline Bern. . . & 148 & 12 & 0,95 \\
\hline Davos.. & 78 & 10 & 0,93 \\
\hline Lausanne . & 117 & 12 & 0,76 \\
\hline Locarno-Monti & 186 & 23 & 0,69 \\
\hline Neuchâtel . & 107 & 8 & 0,80 \\
\hline Pratteln. & (115) & 8 & 0,93 \\
\hline Rorschach : & 136 & 10 & 1,13 \\
\hline St.Gallen . & 138 & 14 & 1,06 \\
\hline Schaffhausen & 120 & 10 & 1,08 \\
\hline $\left.\operatorname{Sion}^{1}\right) .$. & 52 & (8) & $(0,76)$ \\
\hline Thun ... & 134 & 14 & 0,96 \\
\hline Uster & 136 & 10 & 0,97 \\
\hline Zürich . . . & 132 & 8 & 0,75 \\
\hline Bregenz . . & 150 & 15 & 1,00 \\
\hline Como . . . & 151 & 27 & 0,75 \\
\hline \multicolumn{4}{|c|}{$\begin{array}{l}\text { 1) Sion: Der Wert } B \text { variiert mit der Häufigkeit und de } \\
\text { Regendauer }(B=8 \text { min gilt für } z=1) \text {. Der Häufigkeitsfakto } \\
\text { variiert mit der Regendauer }(C=0,76 \text { gilt für } T=15 \mathrm{~min}) \text {. }\end{array}$} \\
\hline
\end{tabular}

Für die Stationen im schweizerischen Mittelland streuen diese Werte zwischen I07 l/s ha für Neuchâtel und I48 l/s ha für Bern. Extrem niedrige Werte weisen Sion ( $52 \mathrm{l} / \mathrm{s}$ ha) und Davos ( $78 \mathrm{l} / \mathrm{s}$ ha) auf. Der höchste Wert wurde für Locarno mit $186 \mathrm{l} / \mathrm{s}$ ha erhalten.

\subsection{Die Zeitbeimerte $\varphi$}

Da diese Werte definitionsgemäss für eine Regendauer $T$ von IS Minuten, die Zahl Eins annehmen müssen, so lautet die Gleichung hierfür gemäss vorstehenden Berechnungen und Überlegungen

$$
\varphi=\frac{15+B}{T+B} .
$$

Die Konstanten $B$ für die einzelnen Stationen, die die Zeitbeiwerte für jede beliebige Regendauer $T$ in Minuten zu errechnen gestatten, sind in Tab. 5 aufgeführt.

Die Zeitbeiwertkurven (und somit auch die Regenintensitätskurven) verlaufen um so flacher, je grösser die Werte $B$ sind. In der Abbildung 8 sind die Regenintensitätskurven, die durchschnittlich pro Jahr einmal erreicht oder überschritten werden, für die einzelnen Orte dargestellt. Sehr flach ist die Kurve von Como, währenddem sich keine ausgesprochen 


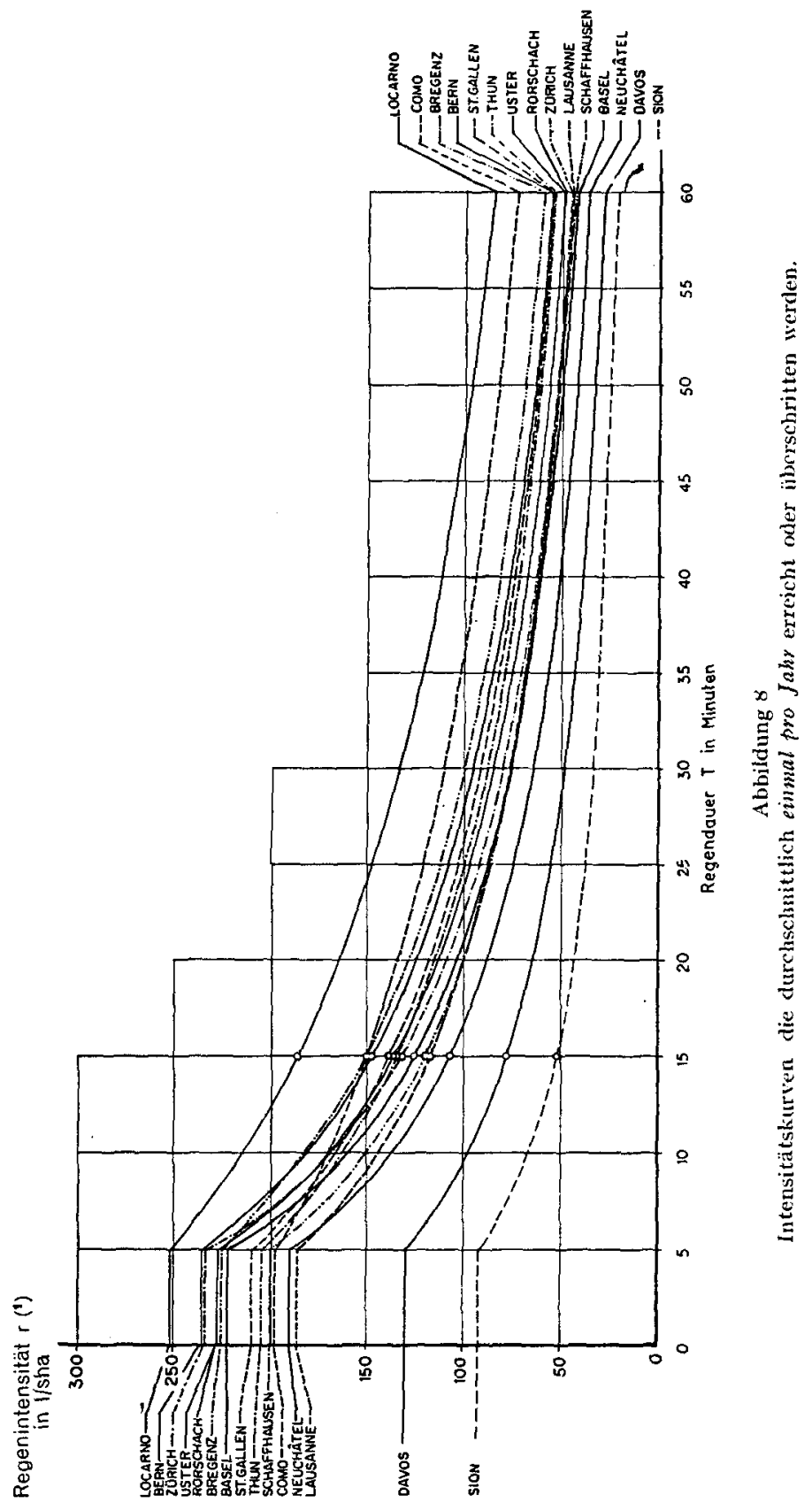


steile Kurve für eine der untersuchten Stationen ergeben hat. Relativ steil sind jedoch die Kurven der Stationen Basel, Neuchâtel, Sion und Zürich. Die Normalwerte von $B$ liegen zwischen 8 und I2 Minuten. Aus den Auswertungen geht hervor, dass für eine Regendauer zwischen $s$ und 60 Minuten keine Potenzformel für den Zeitbeiwert abgeleitet werden kann. Zu diesem Ergebnis gelangte bereits REINHOLD, der für $B$ den Wert 9 für ganz Deutschland fand [5]. Auch in den Vereinigten Staaten werden meistens Regenintensitätsformeln verwendet, die einen gleichen Aufbau aufweisen [I7]. Ebenso ergab die Auswertung der Regenintensitäten für Buenos Aires [I8] den nämlichen Aufbau der Intensitätsgleichung.

\subsection{Die Häufigkeitsfaktoren $h$ - Näberungsformel für die Praxis}

Die Gleichung für die Häufigkeitsfaktoren $b$ erweist sich für den praktischen Gebrauch am unhandlichsten, da diese Faktoren aus einer schiefen Gaußschen Verteilungsfunktion hervorgehen. Die Werte können jedoch mit genügender Genauigkeit aus der Abb. 9 herausgelesen werden. Für den praktischen Gebrauch wurde der Wert $b$ definitionsgemäss so gewählt, dass der einjährigen Regenintensität $(z=\mathbf{I})$ ein Wert $b=\mathbf{I}$ entspricht (siehe Abschnitt 5.4 Faktor $\mu$ ).

Es zeigt sich, dass die in Anlehinung an die Formel von Fuller für die Ermittlung von Hochwassern in Flüssen aufgestellte Beziehung

$$
b=\mathrm{I}+C \log z
$$

innerhalb gewisser Bereiche gute Annäherungswerte an den wahrscheinlichsten Funktionsverlauf ergibt. In der nachfolgenden Tab. 6 sollen deshalb die Konstanten $C$ für jede Meßstation gesondert angegeben werden, zugleich mit Angabe der Fehler gegenüber den rechnerischen Werten für verschiedene Grössen von $z$.

\subsection{Tabelle der Regenintensitäten für den praktiscben Gebrauch}

Um in der Praxis besonders bequem rechnen zu können, fasst man mit Vorteil die Zahlen $G, B+\mathrm{I} 5$ und $b$ in der Weise zusammen, dass $K=G(B+\mathrm{I} \zeta) b$. Die Gleichung für die Regenintensität bekommt dadurch folgende, besonders einfache Form:

$$
r=\frac{K}{T+B} \text {. }
$$

Da für ein gegebenes Problem die Häufigkeit der zu erwartenden Überschreitungen gewählt wird und dadurch bekannt ist, lässt sich der Häufigkeitsfaktor und damit der Wert $K$ von Anfang an festlegen. 


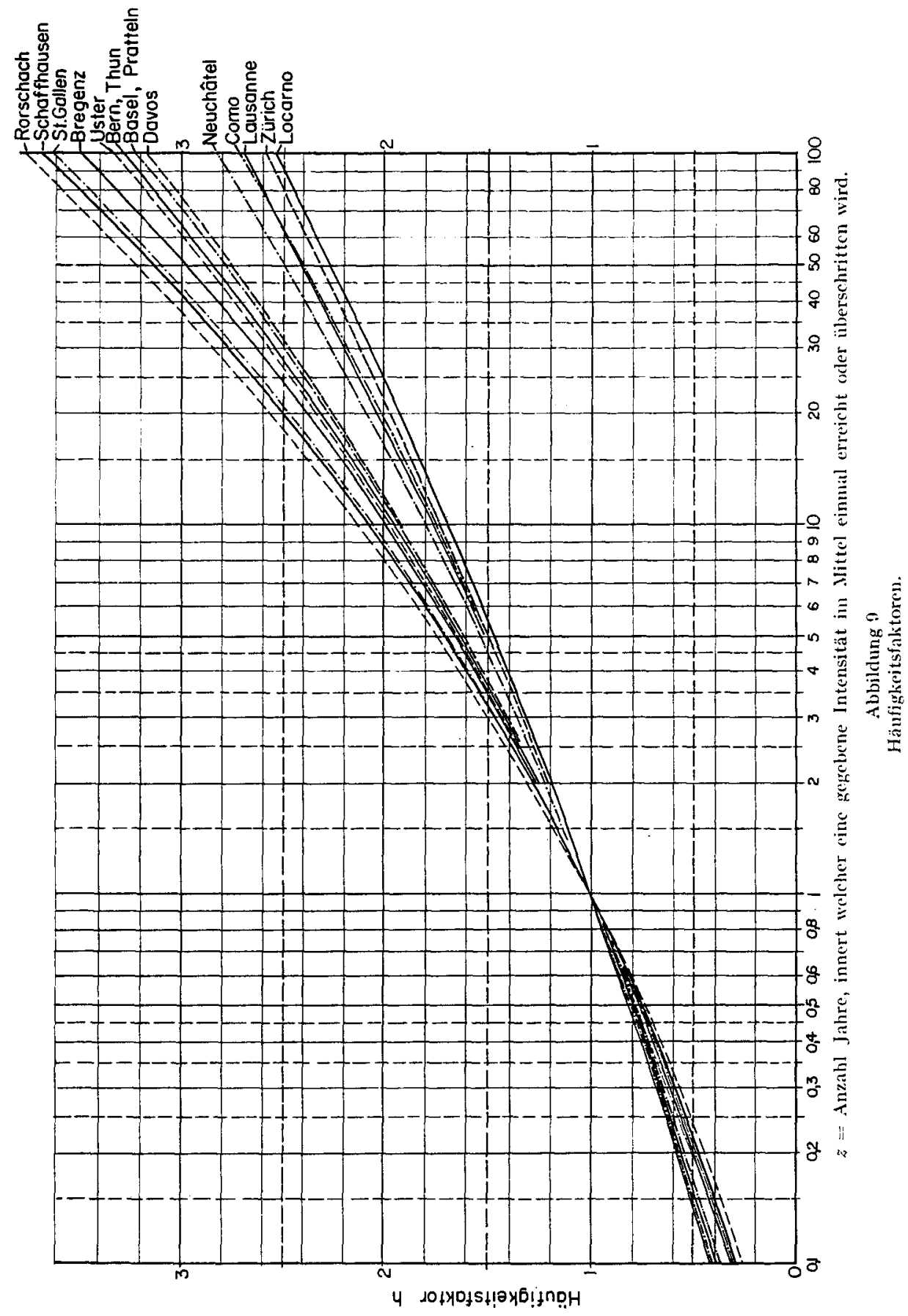


Tabelle 6

Genäherter Häufigkeitsfaktor

Fehler in Prozenten zu wahrscheinlichstem Wert

\begin{tabular}{|c|c|c|c|c|c|c|c|}
\hline \multirow{2}{*}{ Ort } & \multirow{2}{*}{$C$} & \multicolumn{6}{|c|}{ Häufigkeit in $z$ Jahren } \\
\hline & & 1 & 2 & 5 & 10 & 15 & 20 \\
\hline Basel & 0,93 & 0 & $+2,5$ & $+1,9$ & 0 & $-1,9$ & $-2,6$ \\
\hline Bern. & 0,95 & 0 & $+3,2$ & $+2,5$ & 0 & $-1,8$ & $-2,6$ \\
\hline Davos. . & 0,93 & 0 & $+2,4$ & $+1,8$ & 0 & $-1,4$ & $-1,8$ \\
\hline Lausanne. . & 0,76 & 0 & $+1,9$ & $+1,3$ & 0 & $-1,2$ & $-2,0$ \\
\hline Locarno-Monti & 0,69 & 0 & $+1,7$ & $+0,7$ & 0 & $-1,1$ & $-1,9$ \\
\hline Neuchâtel . & 0,80 & 0 & $+2,5$ & $+2,0$ & 0 & $-1,0$ & $-1,9$ \\
\hline Pratteln . & 0,93 & 0 & $+2,4$ & $+2,0$ & 0 & $-2,0$ & $-2,5$ \\
\hline Rorschach & 1,13 & 0 & $+3,4$ & $+2,7$ & 0 & $-2,1$ & $-3,9$ \\
\hline St.Gallen. . & 1,06 & 0 & $+3,1$ & $+2,3$ & 0 & $-2,2$ & $-4,1$ \\
\hline Schaffhausen & 1,08 & 0 & $+3,1$ & $+2,9$ & 0 & $-1,7$ & $-4,0$ \\
\hline Sion. . . . & $(0,76)$ & 0 & - & - & 0 & - & - \\
\hline Thun . . & 0,96 & 0 & $+2,4$ & $+2,3$ & 0 & $-2,9$ & $-\mathbf{3}, 7$ \\
\hline Uster . & 0,97 & 0 & $+2,7$ & $+2,1$ & 0 & $-1,7$ & $-2,8$ \\
\hline Zürich. & 0,75 & 0 & $+1,6$ & $+1,3$ & 0 & $-1,0$ & $-1,9$ \\
\hline Bregenz & 1,00 & 0 & $+2,4$ & $+2,3$ & 0 & $-1,3$ & $-3,8$ \\
\hline Como . . & 0,75 & 0 & $+1,8$ & $+1,7$ & 0 & $-1,0$ & $-1,9$ \\
\hline
\end{tabular}

Tabelle 7

Tabelle der Konstanten $K$

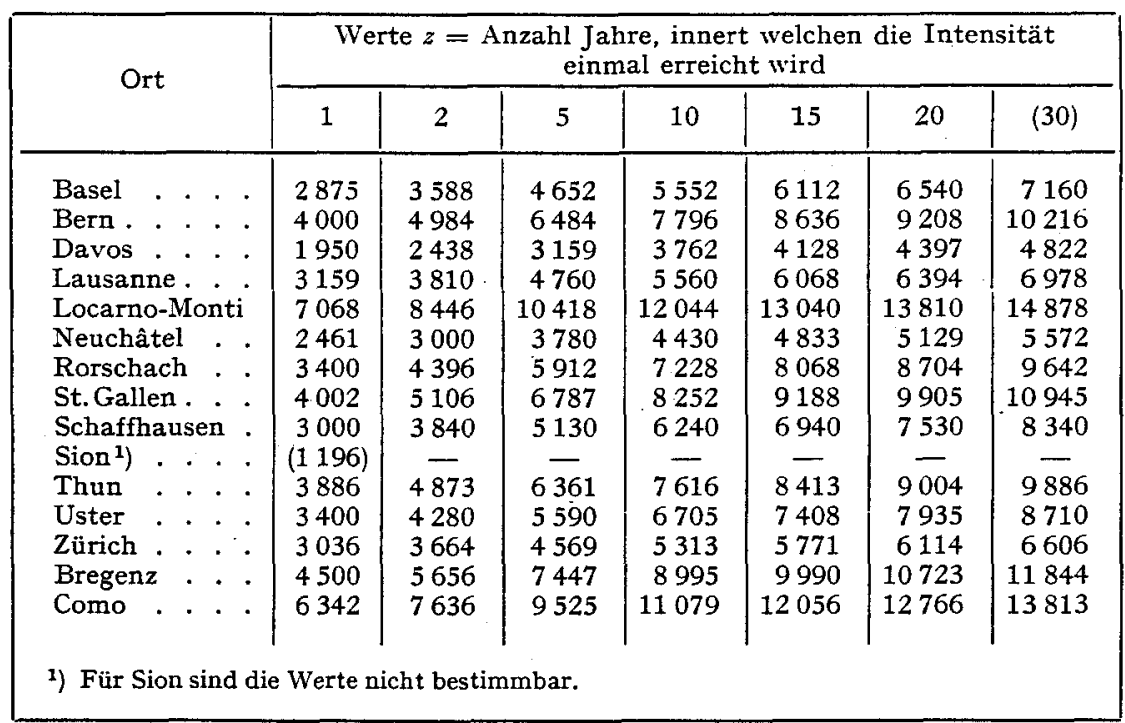


Um den Gebrauch der in dieser Abhandlung vorgelegten Formel für die Regenintensität zu erleichtern, sei nachfolgend in Tab. 7 für alle ausgewerteten Regenmeßstationen (mit Ausnahme von Sion, siehe Bemerkung im Abschnitt 5.2) und für einige ausgewählte Häufigkeiten ( $z=1,2,5$, IO, IS, 20 und 30 Jahre) die Werte $K$ aufgeführt ${ }^{1}$ ).

Damit lässt sich leicht die dem praktisch tätigen Ingenieur gebräuchlichste Darstellung der Regenintensitätskurven, wie sie in Abbildung to am Beispiel der Stadt Bern gezeigt wird, auch für alle andern ausgewerteten Stationen aufstellen.

Der mit der Ausarbeitung eines generellen Kanalisationsprojektes beauftragte Ingenieur möge die Mühe nicht scheuen, vor Inangriffnahme der hydraulischen Berechnung der Kanäle eine Tabelle aufzustellen, die die Beziehung zwischen der Regendauer $T$ und der Regenintensität $r$ für die von ihm gewählten Häufigkeiten der jährlichen Überstauungen wiedergibt. Es hat sich als zweckmässig erwiesen, die Regendauer-

1) Werte $K$ berechnet mit Häufigkeitsfaktor, der auf Gaußscher Verteilungsfunktion beruht.

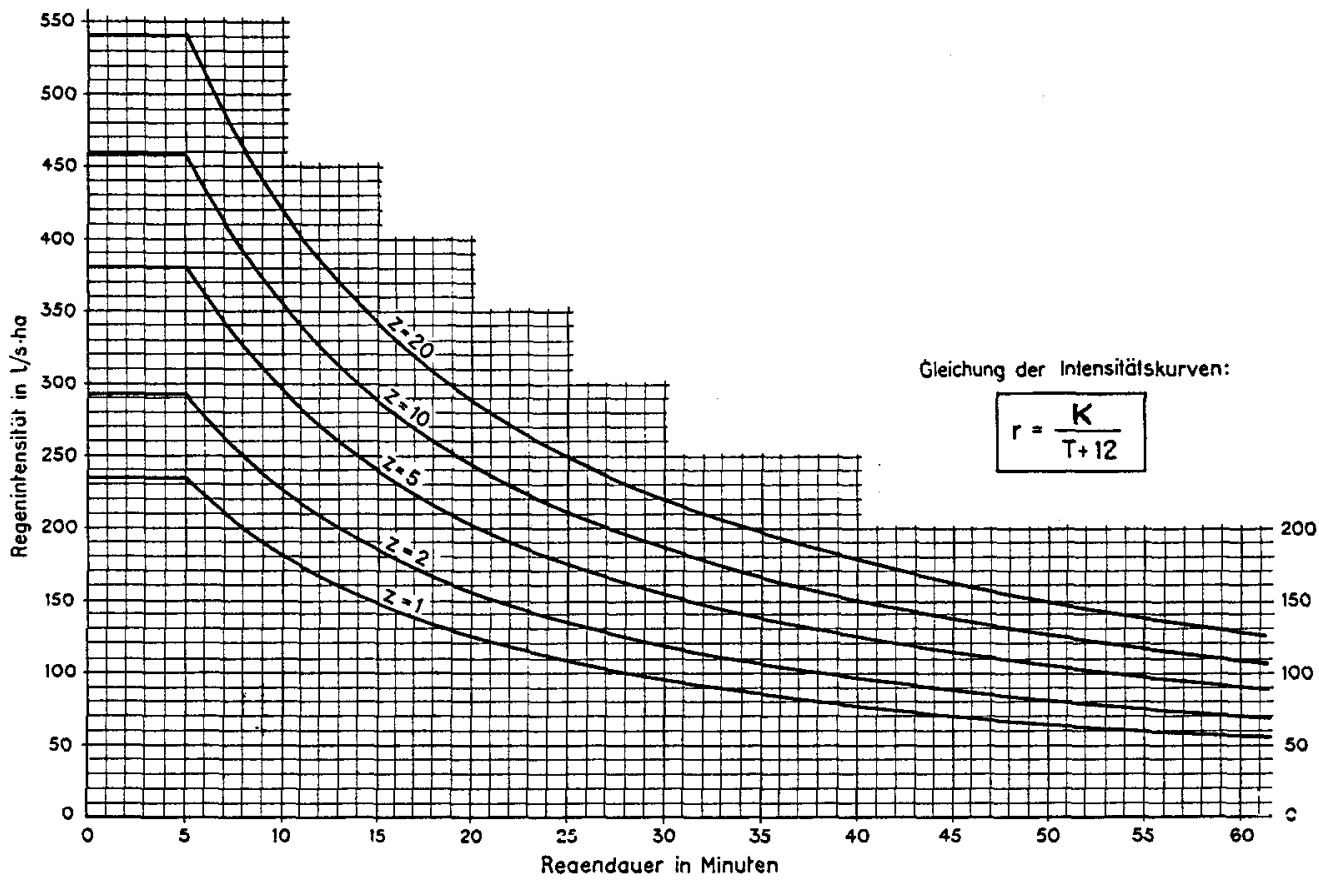

Abbildung 10

Regenintensitätskurven von Bern. 
stufen in dieser Tabelle von Io zu Io Sekunden aufzuführen und auf die Angabe der Laufzeit in Minuten in der Listenrechnung nach Imhoff $z u$ verzichten. Beträgt die mutmasslich grösste Laufzeit inklusive Anlaufszeit rund 20 Minuten, so wird man die Tabelle nur bis zu dieser Regendauer detailliert aufstellen. Für einzelne längere Laufzeiten können die Intensitäten separat errechnet werden. Für die Regenintensitäten der Stadt Zürich wurde beispielsweise eine solche Tabelle bis zu einer Regendauer von $T=30$ Minuten als genügend erachtet.

Als Beispiel diene die Tabelle 8, die den Anfang einer ausführlichen Tabelle für die Station Bern wiedergibt.

Tabelle 8

Beispiel: Regenintensitäten für die Stadt Bern

\begin{tabular}{|c|c|c|c|c|c|c|}
\hline \multicolumn{2}{|c|}{ Regendauer } & \multicolumn{5}{|c|}{$\begin{array}{c}\text { Regenintensität in } 1 / \mathrm{s} \cdot \text { ha für die angegebenen } \\
\text { Häufigkeiten } z \text { in Jahren }\end{array}$} \\
\hline Minuten & Sekunden & 1 & 2 & 5 & 10 & 20 \\
\hline \multirow[t]{6}{*}{5} & 300 & 235 & 293 & 381 & 458 & 542 \\
\hline & 310 & 233 & 290 & 377 & 454 & 536 \\
\hline & 320 & 231 & 287 & 374 & 450 & 531 \\
\hline & 330 & 228 & 284 & 370 & 445 & 526 \\
\hline & 340 & 226 & 282 & 366 & 441 & 521 \\
\hline & 350 & 224 & 279 & 363 & 437 & 516 \\
\hline \multirow[t]{6}{*}{6} & 360 & 222 & 276 & 360 & 433 & 512 \\
\hline & 370 & 220 & 274 & 357 & 429 & 507 \\
\hline & 380 & 218 & 272 & 354 & 425 & 502 \\
\hline & 390 & 216 & 269 & 350 & 421 & 498 \\
\hline & 400 & 214 & 267 & 347 & 418 & 493 \\
\hline & 410 & 212 & 264 & 344 & 414 & 489 \\
\hline \multirow[t]{6}{*}{7} & 420 & 210 & 262 & 341 & 410 & 485 \\
\hline & 430 & 209 & 260 & 338 & 407 & 480 \\
\hline & 440 & 207 & 258 & 335 & 403 & 476 \\
\hline & 450 & 205 & 255 & 332 & 400 & 472 \\
\hline & 460 & 204 & 253 & 329 & 396 & 468 \\
\hline & 470 & 202 & 251 & 326 & 393 & 464 \\
\hline \multirow[t]{6}{*}{8} & 480 & 200 & 249 & 324 & 390 & 460 \\
\hline & 490 & 198 & 247 & 321 & 387 & 457 \\
\hline & 500 & 197 & 245 & 319 & 383 & 453 \\
\hline & 510 & 195 & 243 & 316 & 380 & 449 \\
\hline & 520 & 193 & 241 & 314 & 377 & 446 \\
\hline & 530 & 192 & 239 & 311 & 374 & 442 \\
\hline 9 & 540 & 190 & 237 & 309 & 371 & 438 \\
\hline
\end{tabular}

\section{Schlussbetrachtung}

Aus vorstehenden Ausführungen lassen sich die für die Projektbearbeitung im Gebiete des kommunalen Wasserbaues erforderlichen Starkregenintensitäten für sämtliche Orte der Schweiz ermitteln, die über 


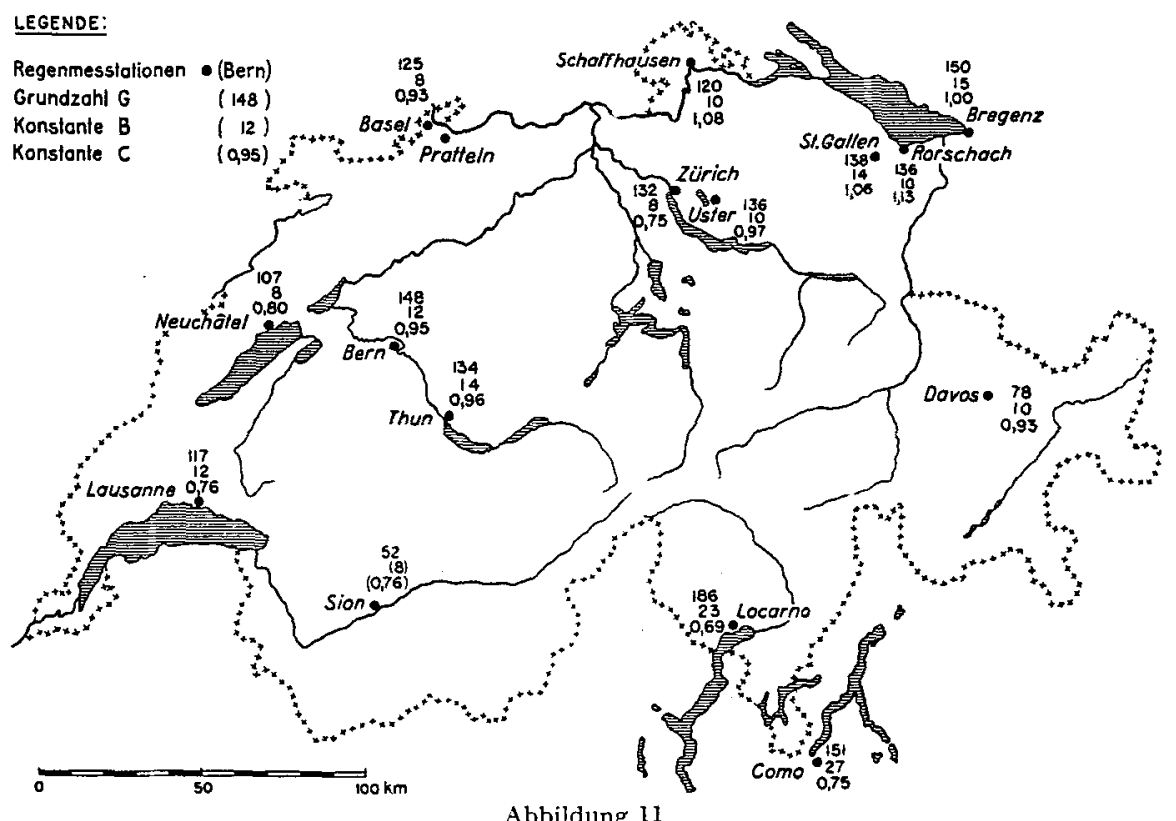

Ausgewertete Regenmeßstationen mit ihren Konstanten (Karte).

langjährige graphische Aufzeichnungen der Regenintensitäten verfügen. Die wesentlichsten Angaben zur Konstruktion der Regenintensitätskurven bestimmter Häufigkeiten gehen aus den Abbildungen 8 und 9 hervor. Während für weitere Studien die Tabellen 3 der Häufigkeitsverteilung von Bedeutung sind, wird der praktisch tätige Ingenieur mit Vorteil die Abbildung II benützen.

Oft stellt sich jedoch die Frage, wie für andere Ortschaften der Schweiz, die keinen Schreibregenmesser besitzen oder die erst seit wenigen Jahren einen Schreibregenmesser in den Dienst nahmen, die Starkregenintensitäten gewählt werden müssen. Aus der Abbildung 8 geht hervor, dass die Schweiz, in Hinsicht auf die Intensitätskurven, die durchschnittlich einmal pro Jahr erreicht oder überschritten werden, in drei Gebiete unterteilt werden kann:

I. In das Gebiet südlich der Alpen, wo für den Südfuss der Alpen (Locarno) extrem hohe Intensitäten festgestellt wurden.

2. In das Gebiet des schweizerischen Mittellandes, wobei die Gebiete der Westschweiz (Neuchâtel, Lausanne) die untere Begrenzung der Intensitäten des Mittellandes ergeben.

3. In die Alpenlängstäler mit extrem geringen Intensitäten (Davos, Sion). 
Aus den Häufigkeitsfaktoren der Abbildung 9 geht die obenerwähnte Unterteilung nicht hervor. Immerhin scheint es, dass die Häufigkeitsfaktoren der Nordostschweiz durchgehend die höchsten Werte aufweisen.

Um die Starkregenintensitäten für einen beliebigen Ort der Schweiz zu ermitteln, ist es vorerst kaum anders möglich, als die Intensitätskurven durch Interpolation zu wählen, wobei den örtlichen topographischen und meteorologischen Gegebenheiten angemessene Rechnung zu tragen ist. Je dichter das Beobachtungsnetz und je einfacher die Terraingestaltung des Gebietes ist, um so weniger sind durch solche Annahmen Überraschungen zu gewärtigen.

Bei dieser Sachlage wird man versuchen müssen, in einigen Jahren ein dichteres Beobachtungsnetz für die Schweiz zu erlangen. Namentlich im Jura, im Voralpengebiet und im südlichen Tessin ist die Erstellung weiterer Schreibregenmesser dringend erwünscht. Aber auch im schweizerischen Mittelland weisen die Beobachtungen noch beachtliche Lücken auf.

Wir richten deshalb an alle Gewässerschutzkreise den Appell, die Erstellung von Registrierstationen für Niederschläge und auch für Abflüsse im Rahmen ihrer Möglichkeiten zu unterstützen und zu fördern. 
Anhang

\begin{tabular}{|c|c|c|c|c|c|c|c|c|c|c|}
\hline \multicolumn{11}{|c|}{ Umkehrfunktion $\Psi(P)$} \\
\hline \multicolumn{11}{|c|}{$0,01 \leq 100 P \leq 1,09$} \\
\hline $100 P$ & 0,00 & 0,01 & 0,02 & 0,03 & 0,04 & 0,05 & 0,06 & 0,07 & 0,08 & 0,09 \\
\hline $\begin{array}{l}0,0 \\
0,1 \\
0,2 \\
0,3 \\
0,4 \\
0,5 \\
0,6 \\
0,7 \\
0,8 \\
0,9 \\
1,0\end{array}$ & $\begin{array}{l}- \\
3,090 \\
2,878 \\
2,748 \\
2,652 \\
2,576 \\
2,512 \\
2,457 \\
2,409 \\
2,366 \\
2,326\end{array}$ & $\begin{array}{l}3,719 \\
3,062 \\
2,863 \\
2,737 \\
2,644 \\
2,569 \\
2,506 \\
2,452 \\
2,404 \\
2,362 \\
2,323\end{array}$ & $\begin{array}{l}3,540 \\
3,036 \\
2,848 \\
2,727 \\
2,636 \\
2,562 \\
2,501 \\
2,447 \\
2,400 \\
2,357 \\
2,319\end{array}$ & $\begin{array}{l}3,432 \\
3,011 \\
2,834 \\
2,716 \\
2,628 \\
2,556 \\
2,495 \\
2,442 \\
2,395 \\
2,353 \\
2,315\end{array}$ & $\begin{array}{l}3,353 \\
2,989 \\
2,820 \\
2,706 \\
2,620 \\
2,549 \\
2,489 \\
2,437 \\
2,391 \\
2,349 \\
2,312\end{array}$ & $\begin{array}{l}3,291 \\
2,968 \\
2,807 \\
2,697 \\
2,612 \\
2,543 \\
2,484 \\
2,432 \\
2,387 \\
2,346 \\
2,308\end{array}$ & $\begin{array}{l}3,239 \\
2,948 \\
2,794 \\
2,687 \\
2,605 \\
2,536 \\
2,478 \\
2,428 \\
2,382 \\
2,342 \\
2,304\end{array}$ & $\begin{array}{l}3,195 \\
2,929 \\
2,782 \\
2,678 \\
2,597 \\
2,530 \\
2,473 \\
2,423 \\
2,378 \\
2,338 \\
2,301\end{array}$ & $\begin{array}{l}3,156 \\
2,911 \\
2,770 \\
2,669 \\
2,590 \\
2,524 \\
2,468 \\
2,418 \\
2,374 \\
2,334 \\
2,297\end{array}$ & $\begin{array}{l}3,121 \\
2,894 \\
2,759 \\
2,661 \\
2,583 \\
2,518 \\
2,462 \\
2,414 \\
2,370 \\
2,330 \\
2,294\end{array}$ \\
\hline \multicolumn{11}{|c|}{$1,0 \leq 100 P \leq 10,9$} \\
\hline $100 P$ & 0,0 & 0,1 & 0,2 & 0,3 & 0,4 & 0,5 & 0,6 & 0,7 & 0,8 & 0,9 \\
\hline $\begin{array}{r}1 \\
2 \\
3 \\
4 \\
5 \\
6 \\
7 \\
8 \\
9 \\
10\end{array}$ & $\begin{array}{l}2,326 \\
2,054 \\
1,881 \\
1,751 \\
1,645 \\
1,555 \\
1,476 \\
1,405 \\
1,341 \\
1,282\end{array}$ & $\begin{array}{l}2,290 \\
2,034 \\
1,866 \\
1,739 \\
1,635 \\
1,546 \\
1,468 \\
1,398 \\
1,335 \\
1,276\end{array}$ & $\begin{array}{l}2,257 \\
2,014 \\
1,852 \\
1,728 \\
1,626 \\
1,538 \\
1,461 \\
1,392 \\
1,329 \\
1,270\end{array}$ & $\begin{array}{l}2,226 \\
1,995 \\
1,838 \\
1,717 \\
1,616 \\
1,530 \\
1,454 \\
1,385 \\
1,323 \\
1,265\end{array}$ & $\begin{array}{l}2,197 \\
1,977 \\
1,825 \\
1,706 \\
1,607 \\
1,522 \\
1,447 \\
1,379 \\
1,317 \\
1,259\end{array}$ & $\begin{array}{l}2,170 \\
1,960 \\
1,812 \\
1,695 \\
1,598 \\
1,514 \\
1,440 \\
1,372 \\
1,311 \\
1,254\end{array}$ & $\begin{array}{l}2,144 \\
1,943 \\
1,799 \\
1,685 \\
1,589 \\
1,506 \\
1,433 \\
1,366 \\
1,305 \\
1,248\end{array}$ & $\begin{array}{l}2,120 \\
1,927 \\
1,787 \\
1,675 \\
1,580 \\
1,499 \\
1,426 \\
1,359 \\
1,299 \\
1,243\end{array}$ & $\begin{array}{l}2,097 \\
1,911 \\
1,774 \\
1,665 \\
1,572 \\
1,491 \\
1,419 \\
1,353 \\
1,293 \\
1,237\end{array}$ & $\begin{array}{l}2,075 \\
1,896 \\
1,762 \\
1,655 \\
1,563 \\
1,483 \\
1,412 \\
1,347 \\
1,287 \\
1,232\end{array}$ \\
\hline \multicolumn{11}{|c|}{$10 \leq 100 P \leq 49$} \\
\hline $100 P$ & 0 & 1 & 2 & 3 & 4 & 5 & 6 & 7 & 8 & 9 \\
\hline $\begin{array}{l}10 \\
20 \\
30 \\
40\end{array}$ & $\begin{array}{l}1,282 \\
0,842 \\
0,524 \\
0,253\end{array}$ & $\begin{array}{l}1,227 \\
0,806 \\
0,496 \\
0,228\end{array}$ & $\begin{array}{l}1,175 \\
0,772 \\
0,468 \\
0,202\end{array}$ & $\begin{array}{l}1,126 \\
0,739 \\
0,440 \\
0,176\end{array}$ & $\begin{array}{l}1,080 \\
0,706 \\
0,412 \\
0,151\end{array}$ & $\begin{array}{l}1,036 \\
0,674 \\
0,385 \\
0,126\end{array}$ & $\begin{array}{l}0,994 \\
0,643 \\
0,358 \\
0,100\end{array}$ & $\begin{array}{l}0,954 \\
0,613 \\
0,332 \\
0,075\end{array}$ & $\begin{array}{l}0,915 \\
0,583 \\
0,305 \\
0,050\end{array}$ & $\begin{array}{l}0,878 \\
0,553 \\
0,279 \\
0,025\end{array}$ \\
\hline $\begin{array}{l}\mathrm{Me} \\
\mathrm{F} \ddot{\mathrm{u}} \\
\mathrm{Vo}\end{array}$ & 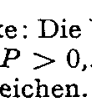 & & 0 & 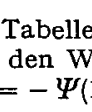 & & 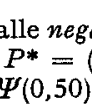 & $=0$. & & & \\
\hline
\end{tabular}




\section{LITERATURVERZEICHNIS}

[I] Kropf, A., Auswertung von zojäbrigen Aufzeichnungen der Regenmess-Stution der $M Z A$ in Züricb, Schweiz. Bauzeitung 123 (1944) 225 .

[2] Hörler, A., Auswertung von 3ojäbrigen Aufzeichnungen der Regenmess-Station Rotmonten in St. Gallen, Schweiz. Z. Hydrol. 20 (1958) Fasc. I.

[3] Wegenstein, M., Grundlagen der Ortsentwässerung. Strasse und Verkebr.

[4] Abwasserfachgruppe der Deutschen Gesellschaft für Bauwesen e. V., Anmeisungen zur Auswertung von Scbreibregenmesser-Aufzeicbnungen für wasserwirtscbaftlicbe Znecke (AAR 1936), Ges.-Ing. 60 (I937), H. 2, 22-26; H. 3. 40-65; H. 4, 55-6I.

[s] Reinhold, F., Regenspenden in Deutschland (Grundwerte für die Entwässerungstechnik). Arch. f. Wasserwirtsch., Nr. 56 (Berlin 1940).

[6] VEN TE CHOw, Frequency Analysis of Hydrologic Data mith Special Application to Rainfall Intensities, Univ. of Illinois Bulletin, Chicago (July I953).

[7] MeYER, A. F., The Elements of Hydrology (John Wiley \& Sons, New York 1928).

[8] FAIR, G. M., and GeYer, J. C., Water Supply and Waste-Water Disposal (John Wiley \& Sons, New York 1954).

[9] Johannsen, H. H., Eine einfache Darstellung der Ergebnisse pon SchreibregenmesserAufzeichnungen, Ber. d. Deutsch. Wetterdienstes, Nr. 54 (1959).

[Io] SLADE, J. J., The Reliability of Statistical Metbods in the Determination of Flood Frequencies. U.S. Geological Survey, Water Supply Paper 771 (1936).

[I] HaLd, A., Statistical Theory with Engineering Applications (John Wiley \& Sons, New York 1957).

[12] HaLD, A., Statistical Tables and Formulas (John Wiley \& Sons, New York 1958).

[13] VAN DER WAERden, B. L., Matbematische Statistik (Springer-Verlag, Berlin I957).

[14] Fisher, R. A., and Yates, F., Statistical Tables for Biological, Agricultural and Medical Research (Oliver and Boyd, London 1957).

[15] Goodrich, R. D., Straight line plotting of skew frequency data, Proc. Am. Soc. Civ. Eng. 52 (1926), 1063.

[16] IмноFF, K., Taschenbuch der Stadtentwässerung. I 8. Auf. (Oldenbourg-Verlag, München 1960).

[17] Davis, C. V., Handbook of Applied Hydraulics (McGraw-Hill Book Comp., New York 1952).

[18] MARCHETTI, A. A., Frecuencia de las Llupias intensas de corta duración en la ciudat de Buenos Aires. Revista Meteoros, 2, Nr. I-2 (Januar-Juni 1952).

[19] RIMA, A., Intensità della pioggia della città di Locarno, Legge delle intensità massime. Wasser- und Energiewirtschaft 51 (1959), Nr. 6.

[20] RIMA, A., Intensità della pioggia per Chiasso e dintorni, Legge delle intensità massime assolute (dedotta dalle registrazioni pludiografiche di Como). Rivista tecnica della Svizzera Italiana (I96r), Nr. I6.

[2I] HörLer, A. und RHEIN, H. R., Die Intensitäten der Starkregen in der Scbweiz, Schweiz. Bauzeitung 79 (1961), H. 32, 559. 